\title{
Development of a Performance Estimation Tool for Gas Turbine Engine Centrifugal Compressors
}

by

\author{
Ian A.W. Britton \\ B.Sc.E. (Mechanical) \\ A thesis submitted to \\ the Faculty of Graduate Studies and Research \\ in partial fulfillment of the requirements \\ for the degree of
}

\author{
Master of Applied Science \\ in \\ Mechanical Engineering \\ Ottawa-Carleton Institute For \\ Mechanical and Aerospace Engineering
}

Department of Mechanical and Aerospace Engineering

Carleton University

Ottawa, Ontario Canada

January 2008

(C) 2008

Ian A.W. Britton 


$\begin{array}{ll}\begin{array}{l}\text { Library and } \\ \text { Archives Canada }\end{array} & \begin{array}{l}\text { Bibliothèque et } \\ \text { Archives Canada }\end{array} \\ \begin{array}{l}\text { Published Heritage } \\ \text { Branch }\end{array} & \begin{array}{l}\text { Direction du } \\ \text { Patrimoine de l'édition }\end{array} \\ \begin{array}{l}\text { 395 Wellington Street } \\ \text { Ottawa ON K1A 0N4 } \\ \text { Canada }\end{array} & \begin{array}{l}\text { 395, rue Wellington } \\ \text { Ottawa ON K1A 0N4 } \\ \text { Canada }\end{array}\end{array}$

Your file Votre référence ISBN: 978-0-494-40632-8

Our file Notre référence

ISBN: 978-0-494-40632-8

NOTICE:

The author has granted a nonexclusive license allowing Library and Archives Canada to reproduce, publish, archive, preserve, conserve, communicate to the public by telecommunication or on the Internet, loan, distribute and sell theses worldwide, for commercial or noncommercial purposes, in microform, paper, electronic and/or any other formats.

The author retains copyright ownership and moral rights in this thesis. Neither the thesis nor substantial extracts from it may be printed or otherwise reproduced without the author's permission.
AVIS:

L'auteur a accordé une licence non exclusive permettant à la Bibliothèque et Archives Canada de reproduire, publier, archiver, sauvegarder, conserver, transmettre au public par télécommunication ou par l'Internet, prêter, distribuer et vendre des thèses partout dans le monde, à des fins commerciales ou autres, sur support microforme, papier, électronique et/ou autres formats.

L'auteur conserve la propriété du droit d'auteur et des droits moraux qui protège cette thèse. $\mathrm{Ni}$ la thèse ni des extraits substantiels de celle-ci ne doivent être imprimés ou autrement reproduits sans son autorisation.
In compliance with the Canadian Privacy Act some supporting forms may have been removed from this thesis.

While these forms may be included in the document page count, their removal does not represent any loss of content from the thesis.
Conformément à la loi canadienne sur la protection de la vie privée, quelques formulaires secondaires ont été enlevés de cette thèse.

Bien que ces formulaires aient inclus dans la pagination, il n'y aura aucun contenu manquant.

\section{Canada}




\section{Abstract}

A preliminary design tool has been created to aid in the design and development of a centrifugal flow compressor impeller for a gas turbine engine. The design tool requires basic compressor design point geometry which could be obtained from simple calculations during the preliminary design phase of a gas turbine engine. Using this information, the design tool is able to estimate the compressor map. Elements from several models are incorporated in the design tool, most notably the impeller two-zone model for the construction of the compressor map at higher rotational speeds. To complete the map, the developed tool utilizes a low speed extrapolation technique to estimate low speed characteristics. The two-zone model utilizes few empirical correlations, however those used represent the most appropriate available. Furthermore, the design tool estimates the gas turbine performance based upon the design point data of the compressor, most notably the design pressure ratio and design mass flow rate. From this, component matching calculations are performed to estimate the gas turbine performance over a range of mass flow rates and rotational speeds.

The tool was validated against several test impellers obtained in the literature and also against a computational fluid dynamics study. The design tool is useful for synthesis of a compressor map which is required for the estimation of off-design engine performance at the preliminary design phase. One of the strengths of the design tool is that it allows for compressor characteristics estimation without the requirement for more complex streamline curvature or computational fluid dynamics studies. Overall, the developed tool showed good agreement between the developed compressor maps and actual compressor maps obtained in the literature. Furthermore, the design tool showed good agreement in the overall engine performance calculations when compared to more popular gas turbine engine performance estimation packages. 


\section{Acknowledgements}

The author wishes to acknowledge the support of Professor J.E.D. Gauthier for his invaluable guidance and insight during the course of this research work. Furthermore, the author wishes to thank the opinions and information received from Professor S.A. Sjolander, without which, the project would not have taken shape the way it did. 


\section{Table of Contents}

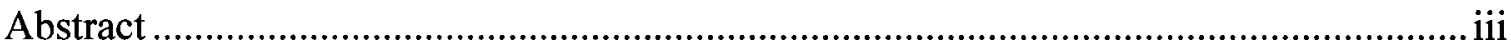

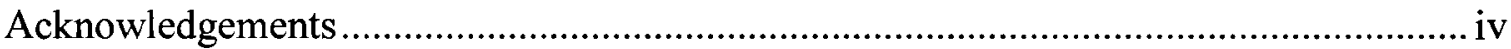

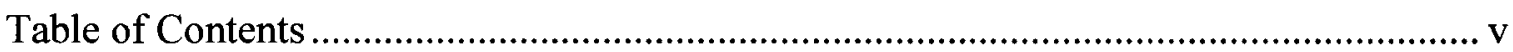

List of Figures .................................................................................................... vii

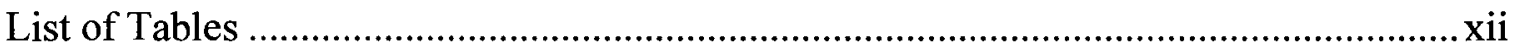

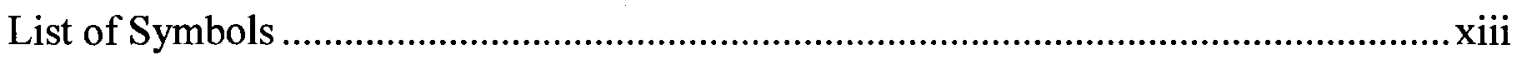

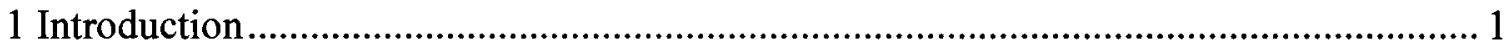

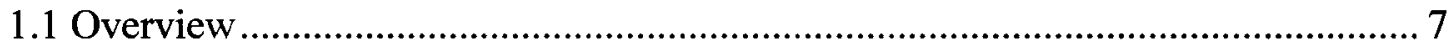

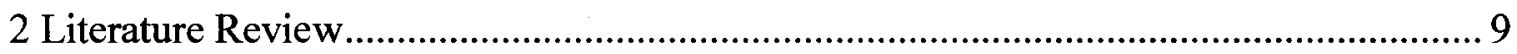

2.1 Gas Turbine Design Procedure ……………………....................................... 9

2.2 Gas Turbine Engine Off-Design Analysis ......................................................... 14

2.3 Compressor Map Generation .......................................................................... 21

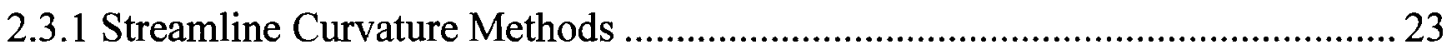

2.3.2 Map Scaling Techniques................................................................................. 27

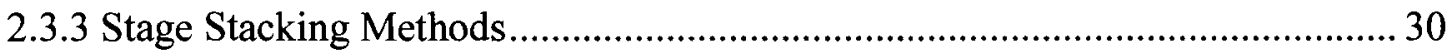

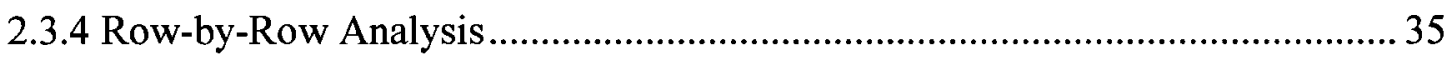

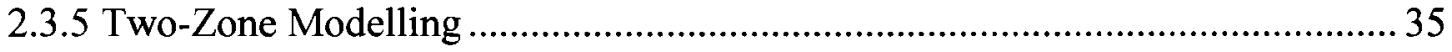

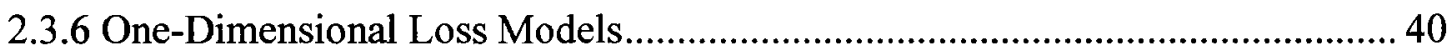

2.3.7 Compressor Map Generation Summary ........................................................... 42

2.4 Turbine Map Generation ................................................................................... 42

2.5 Component Matching Techniques .................................................................... 44

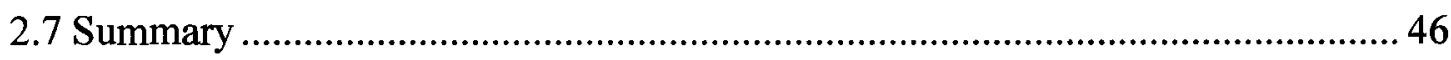

3 Proposed Centrifugal Compressor Map Synthesis Tool and Off-Design Engine

Performance Prediction Tool .............................................................................................. 48

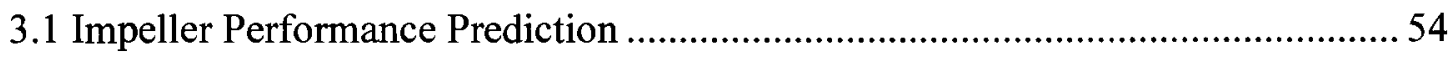

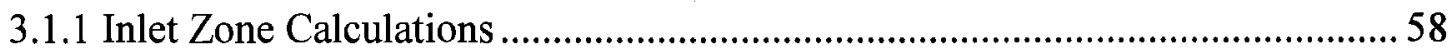

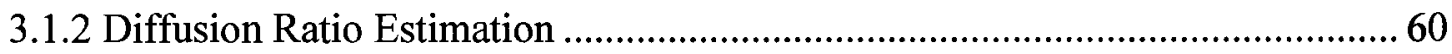

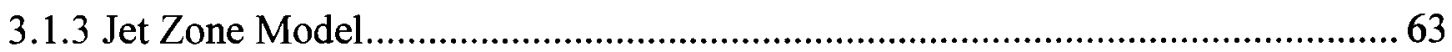




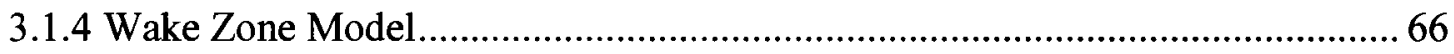

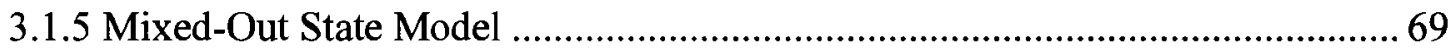

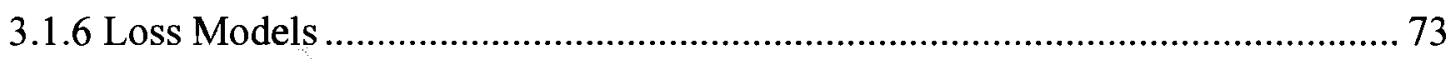

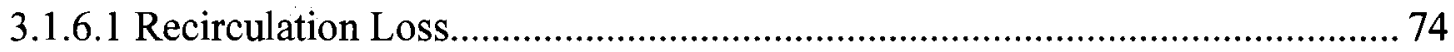

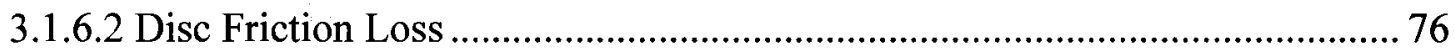

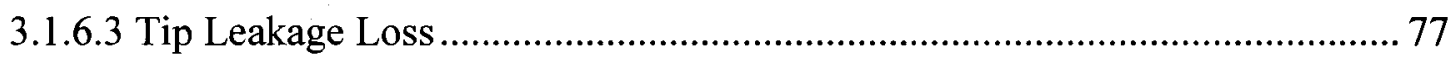

3.1.7 Impeller Model Notes .................................................................................. 79

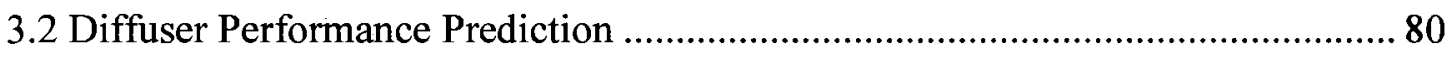

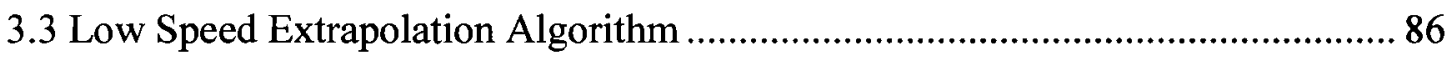

3.4 Beta Grid Definition Algorithm .................................................................. 90

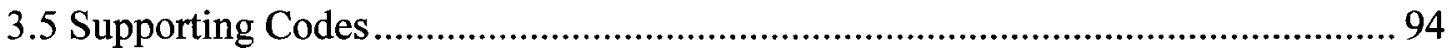

3.5.1 Curve Fitting Algorithm............................................................................... 94

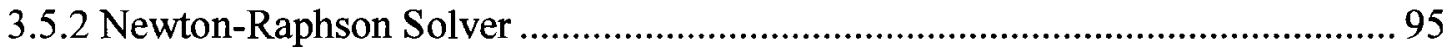

3.6 Component Matching Algorithm ....................................................................... 96

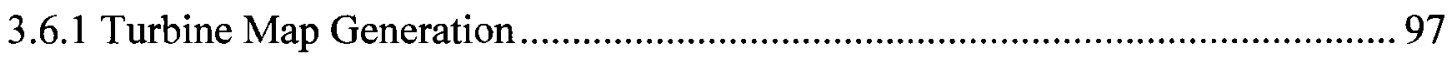

3.6.2 Equilibrium Running Line Determination .......................................................99

3.7 Performance Calculations ............................................................................... 102

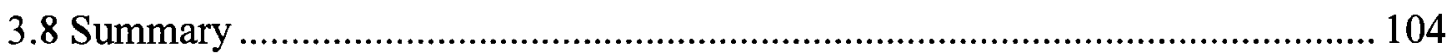

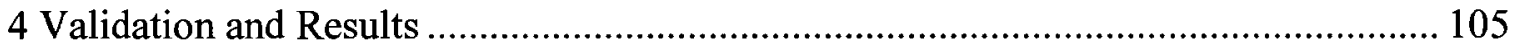

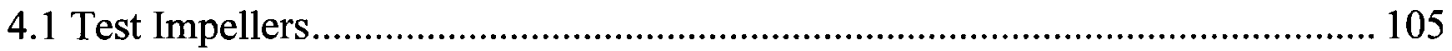

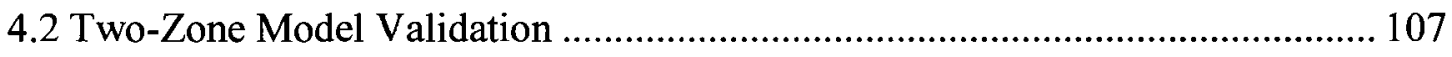

4.3 Low Speed Extrapolation Validation..................................................................... 122

4.4 Performance Prediction Validation ................................................................ 127

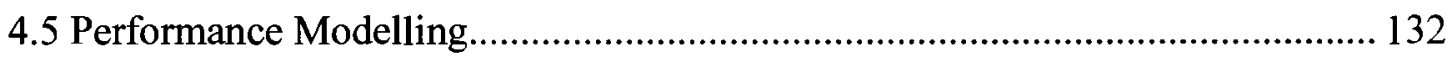

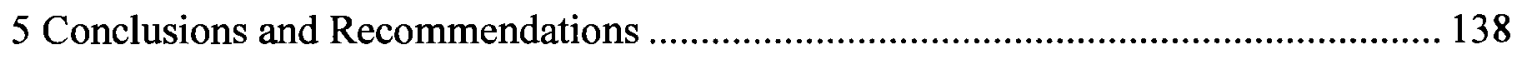

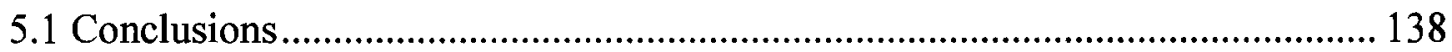

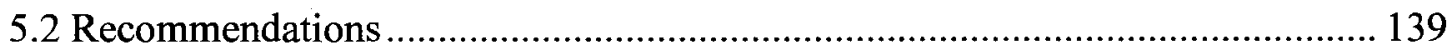

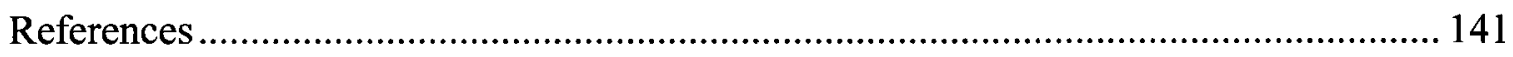

Appendix A - Centrifugal Compressor Map Prediction Tool Source Code.................... 145

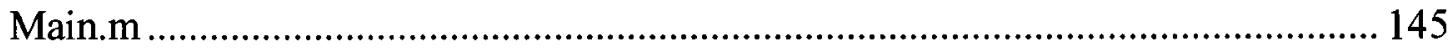

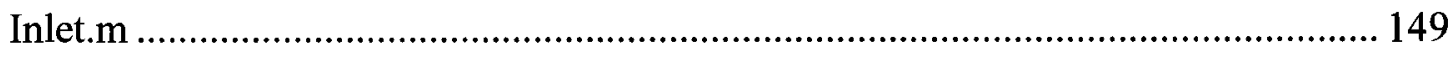




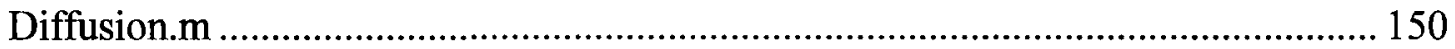

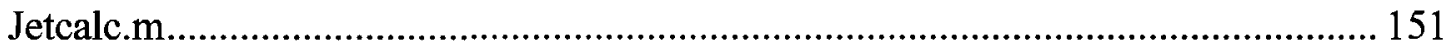

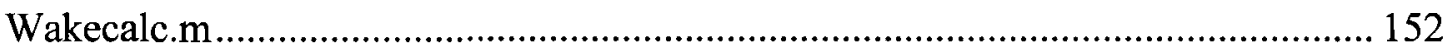

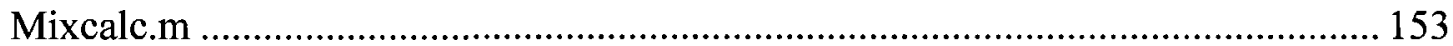

Appendix B - Sample Input Two-Zone Model File ……............................................. 156

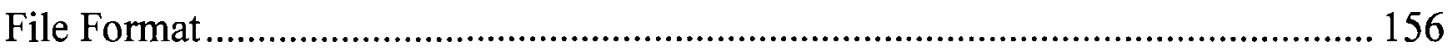

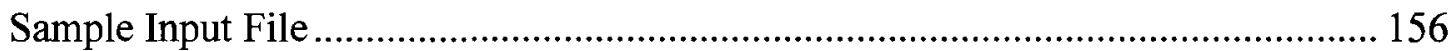

Appendix C - Sample Beta Grid Input File ............................................................. 158

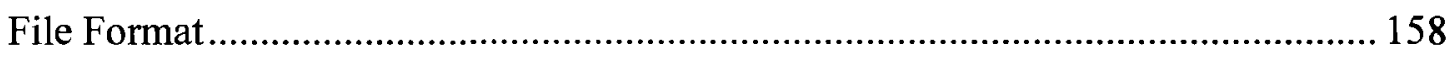

Sample Input File 


\section{List of Figures}

Figure 1.1: Typical compressor map........................................................................... 3

Figure 1.2: Gas turbine engine using a gas generator with a free power turbine................. 5

Figure 1.3: Simple, single spool gas turbine engine schematic. ...................................... 6

Figure 2.1: Typical turbomachinery component design procedure. Based on

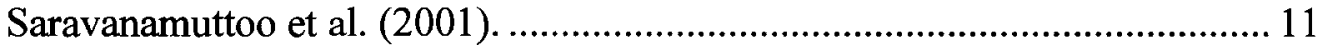

Figure 2.2: Typical compressor performance map. Obtained with the aid of GasTurb ${ }^{\mathrm{TM}} \cdot 16$

Figure 2.3: Typical turbine performance map. Obtained with the aid of GasTurb ${ }^{\mathrm{TM}}$........ 17

Figure 2.4: Equilibrium running line for a typical compressor. Obtained with the aid of

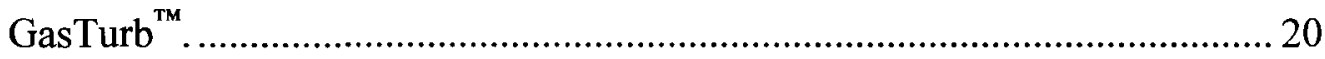

Figure 2.5: Computational domain for the streamline curvature method......................... 24

Figure 2.6: Sample scaled pressure ratio characteristic for use in the compressor map scaling procedure.

Figure 2.7: Generic axial stage compressor characteristic used in the stage stacking method...

Figure 2.8: Schematic of the two-zone model impeller exit flow.

Figure 2.9: Sample turbine map showing the running line established by component matching calculations.

Figure 3.1: Compressor synthesis and gas turbine off-design analysis tool flowchart.... 49

Figure 3.2: Sample data generated by the compressor off design performance model. ... 51

Figure 3.3: Sample beta grid overlaid on a compressor map............................................ 53

Figure 3.4: Schematic of the two-zone model impeller exit flow....................................... 55

Figure 3.5: Impeller model flowchart. ....................................................................... 57

Figure 3.6: Typical beta line grid for use in association with compressor maps.............. 91

Figure 3.7: Beta line algorithm as used in conjunction with the off-design performance code.

Figure 3.8: Normalized turbine characteristic used in the turbine map scaling process... 98 
Figure 3.9: Normalized efficiency characteristic used in the turbine map scaling process.

Figure 4.1: Pressure ratio of Eckhardt impeller O..................................................... 108

Figure 4.2: Isentropic efficiency of Eckhardt impeller O............................................. 108

Figure 4.3: Difference between pressure ratio generated by TZM and the value from the actual impeller map for Eckhardt impeller O............................................... 109

Figure 4.4: Difference between isentropic efficiency generated by TZM and the value from the actual impeller map for Eckhardt impeller O.................................. 109

Figure 4.5: Pressure ratio of Eckhardt impeller A. ........................................................ 111

Figure 4.6: Isentropic efficiency of Eckhardt impeller A. ............................................... 111

Figure 4.7: Difference between pressure rato generated by TZM and the value from the actual impeller map for Eckhardt impeller A.................................................... 112

Figure 4.8: Difference between efficiency generated by TZM and the value from the actual impeller map for Eckhardt impeller A................................................. 112

Figure 4.9: Pressure ratio of the impeller described by McKain and Holbrook (1997). 114

Figure 4.10: Isentropic efficiency of the impeller described by McKain and Holbrook (1997).

Figure 4.11: Difference between the pressure ratio generated by TZM and the value from the actual impeller map for the impeller described by McKain and Holbrook (1997).

Figure 4.12: Difference between the efficiency generated by TZM and the value from the actual impeller map for the impeller described by McKain and Holbrook (1997).

Figure 4.13: Pressure ratio characteristics describing the rotational speed limits of the two-zone model for the impeller described by McKain and Holbrook (1997).

Figure 4.14: Isentropic efficiency characteristics describing the rotational speed limits of the two-zone model for the impeller described by McKain and Holbrook (1997).

Figure 4.15: Pressure ratio of the impeller described by Came and Herbert (1980). ..... 118 
Figure 4.16: Isentropic efficiency of the impeller described by Came and Herbert (1980).

Figure 4.17: Difference between pressure ratio generated by TZM and the value from the actual impeller map described by Came and Herbert (1980).

Figure 4.18: Difference between isentropic efficiency generated by TZM and the value from the actual impeller map described by Came and Herbert (1980). 120

Figure 4.19: Actual map, TZM and LSE pressure ratio characteristics for Eckhardt impeller $O$.

Figure 4.20: Actual map, TZM and LSE efficiency characteristics for Eckhardt impeller O. 124

Figure 4.21: Actual map, TZM and LSE pressure ratio characteristics for Eckhardt impeller $\mathrm{A}$.

Figure 4.22: Actual map, TZM and LSE efficiency characteristics for Eckhardt impeller A.

Figure 4.23: Actual map, TZM and LSE pressure ratio characteristics for the impeller described by Came and Herbert (1980).

Figure 4.24: Actual map, TZM and LSE efficiency characteristics for the impeller described by Came and Herbert (1980).

Figure 4.25: Compressor map obtained from GasTurb for use in the performance prediction algorithm.

Figure 4.26: Equilibrium running lines determined by the GasTurb software package and the in-house code. 129

Figure 4.27: Variation of turbine inlet temperature with net shaft power for a single-spool gas turbine engine.

Figure 4.28: Variation of turbine inlet temperature with rotational speed for a single spool gas turbine engine.

Figure 4.29: Variation of specific fuel consumption with net shaft power for a single spool gas turbine engine.

Figure 4.30: Performance of Eckhardt impeller $O$ using both the actual impeller map and the TZM impeller map. 133 
Figure 4.31: Performance of Eckhardt impeller A using both the actual impeller map and the TZM impeller map. ................................................................................ 134

Figure 4.32: Performance of the impeller described by Came and Herbert (1980) using both the actual impeller map and the TZM impeller map.............................. 134

Figure 4.33: Comparison of SFC calculations based upon the actual maps and the maps obtained by the TZM...................................................................................... 136 


\section{List of Tables}

Table 2.1: Differences between two-zone model methods.............................................. 36

Table 2.2: Benefits and drawbacks to two separate two-zone models................................ 37

Table 3.1: Empirical coefficients for the calculation of diffusion ratio in the TEIS model.

.62

Table 4.1: Specifcations of test impeller used in two-zone model validation. ................ 106

Table 4.2: Data used in GasTurb ${ }^{\mathrm{TM}}$ single shaft engine simulation............................... 128 


\section{List of Symbols}

\section{Variables}

A

$A_{\text {geo }}$

b

B

$\mathrm{C}$

$c_{f}$

$c_{p}$

$C_{p a}$

$C_{p b}$

$C_{p a, i}$

$C_{p b, i}$

D Diameter or divergence parameter (see Eq. 3.97)

$D_{f}$

$D_{m}$

DR

$\mathrm{E}$

$f_{d f}$

$f_{j}$

$\frac{f}{a}$

$\mu_{j}$

$\mathrm{h}$

$\Delta h_{\text {euler }}$

$\Delta H$

Area

Blade height

Blockage (see Eq. 3.84 and 3.92)

Absolute velocity

Skin friction coefficient (see Eq. 3.85 and 3.101)

Specific heat capacity at constant pressure

(see Eq. 3.16)

(see Eq. 3.17)

Empirical diffusion efficiency (see Eq. 3.98)

Backsweep slip modifier (see Eq. 3.27)

Fuel-to-air ratio (see Eq. 3.126)

Jet slip factor (see Eq. 3.28)

Enthalpy

Euler enthalpy change (see Eq. 3.67)

Head change
Impeller exit area excluding blade blockage (see Eq. 3.30b)

Pressure recovery coefficient in the impeller inlet section

Pressure recovery coefficient in the impeller passage section

Ideal pressure recovery coefficient in the impeller inlet

Ideal pressure recovery coefficient of the impeller passage

External loss correlation diffusion factor (see Eq. 3.68)

Limiting value divergence parameter (see Eq. 3.86)

Two-zone model diffusion ratio (see Eq. 3.14, 3.15)

Disc friction correlation friction factor (see Eq. 3.73) (none)

(none)

(none)

$\mathrm{kJ} / \mathrm{kg}$

$\mathrm{kJ} / \mathrm{kg}$

Units

$\mathrm{m}^{2}$

$\mathrm{m}^{2}$

m

(none)

$\mathrm{m} / \mathrm{s}$

(none)

$\mathrm{J} / \mathrm{kgK}$

(none)

(none)

$\mathrm{m}$ or (none)

(none)

(none)

(none)

(none)

(none)

(none)

(none)

$\mathrm{kJ} / \mathrm{kg}$ 


$\begin{array}{llc}\text { HHV } & \text { High heating value (see Eq. 3.126) } & \mathrm{kJ} / \mathrm{kg} \\ \frac{d I_{D}}{d m} & \text { Vaneless diffuser diffusion loss (see Eq. 3.81 and 3.99) } & \mathrm{J} / \mathrm{m} \\ \mathrm{k} & \text { Cube power relationship constant (see Eq. 3.123) } & (\mathrm{none}) \\ \dot{m} & \text { Mass flow rate } & \mathrm{kg} / \mathrm{s} \\ \dot{m}_{\text {air }} & \text { Air mass flow rate (see Eq. 3.127) } & \mathrm{kg} / \mathrm{s} \\ \dot{m}_{\text {fuel }} & \text { Fuel mass flow rate (see Eq. 3.127) } & \mathrm{kg} / \mathrm{s} \\ \mathrm{M} & \text { Mach number } & (\mathrm{none}) \\ \mathrm{m} & \text { Meridional position } & \mathrm{m} \\ \mathrm{N} & \text { Rotational Speed } & \mathrm{rev} / \mathrm{min} \\ \mathrm{P} & \text { Pressure } & \mathrm{kPa} \\ Q & \text { Volumetric flow rate } & \mathrm{m} \text { 3/s } \\ \mathrm{r} & \text { Radius } & \mathrm{m} \\ \mathrm{Re} & \text { Reynolds number (see Eq. 3.72) } & (\mathrm{none}) \\ \mathrm{t} & \text { Thickness } & \mathrm{m} \\ \mathrm{T} & \text { Temperature } & \mathrm{K} \\ \mathrm{U} & \text { Blade speed } & \mathrm{m} / \mathrm{s} \\ \mathrm{w} & \text { Specific work (see Eq 3.107) } & \mathrm{kJ} / \mathrm{kg} \\ \mathrm{W} & \text { Relative flow velocity } & \mathrm{m} / \mathrm{s} \\ \dot{W} & \text { Power (see Eq. 2.16 through 2.18 and 3.109) } & \mathrm{kW} \\ \dot{W}_{\text {net } \text { treq }} & \text { Power requirement (see Eq. 3.124) } & \mathrm{kW} \\ \mathrm{Z} & \text { Number of blades, loss parameter } & (\mathrm{none})\end{array}$

\section{Constants}

$\begin{array}{ll}\pi & \text { Ratio of circle circumference to diameter (approximately } 3.14159 \\ \mathrm{~g} & \text { [dimensionless]) } \\ \mathrm{R} & \text { Gravitational acceleration at sea level }\left(9.81 \mathrm{~m} / \mathrm{s}^{2}\right) \\ & \text { Gas constant for air }(287 \mathrm{~J} / \mathrm{kgK})\end{array}$




\section{Greek Symbols}

$\alpha$

$\beta$

$\gamma$

$\delta$

$\varepsilon$

$\phi$

$\Phi$

$\eta$

$\eta_{\text {comb }}$

$\mu$

$\rho$

$\sigma$

$\theta$

$\psi$

$\omega$

$\zeta$

$x$

\section{Subscripts}

0

1

2

3

4

a

b

bl

c
Absolute flow angle deg.

Relative flow angle deg.

Specific heat ratio (none)

Boundary layer height (see Eq. 3.83 and 3.91) m

Tip clearance or wake area fraction

(see Eq. 3.30 and Eq. 3.75a)

$\mathrm{m}$ or (none)

Flow coefficient (see Eq. 2.5)

(none)

Entropy function (see Eq. 2.10) $\mathrm{kJ} / \mathrm{kgK}$

Isentropic efficiency or effectiveness coefficient (see Eq. 3.66) (none)

Combustion efficiency (see Eq. 3.126) (none)

Viscosity N•s/ $\mathrm{m}^{2}$

Density $\mathrm{kg} / \mathrm{m}^{3}$

Solidity or entropy (none) or $\mathrm{kJ} / \mathrm{kgK}$

Tangential Component

Head or Load coefficient (see Eq. 2.7) (none)

Angular velocity (see Eq. 3.1) $\mathrm{rad} / \mathrm{s}$

Total loss parameter, cube power law coefficient (none)

Wake mass flow fraction (none)

Stagnation condition

Compressor inlet position

Compressor exit position

Turbine inlet position

Turbine exit position

Reference speed line a or inlet effectiveness

Reference speed line $b$ or passage effectiveness

Blade condition

Compressor quantity 


\begin{tabular}{|c|c|}
\hline corr & Corrected map value \\
\hline des & Design point value \\
\hline df & Disc Friction Loss \\
\hline e & Impeller exit condition \\
\hline $\mathrm{fc}$ & Front cover \\
\hline full & Full blade \\
\hline $\mathrm{h}$ & Hub \\
\hline $\mathrm{i}-1$ & Conditions at the previous stage or node \\
\hline $\mathrm{i}$ & Conditions at the current stage or node or ideal condition \\
\hline $\mathrm{i}+1$ & Conditions at the downstream stage or node \\
\hline in & Compressor inlet condition \\
\hline ideal & Ideal condition \\
\hline $\mathrm{j}$ & Jet \\
\hline $\mathrm{m}$ & Meridional component \\
\hline $\operatorname{mix}$ & Mixed-out condition \\
\hline net & Net condition \\
\hline new & New map value \\
\hline normalized & Normalized map value \\
\hline non-normalized & Non-normalized map value \\
\hline orig & Original scaled map value \\
\hline $\mathrm{r}$ & Rotor \\
\hline rc & Recirculation Loss \\
\hline ref & Reference condition \\
\hline s & Isentropic condition or stator \\
\hline scale & Scaled map value \\
\hline split & Splitter blade \\
\hline $\mathrm{t}$ & Turbine quantity or tip condition \\
\hline th & Throat condition \\
\hline $\mathrm{tl}$ & Tip Leakage Loss \\
\hline w & Wake \\
\hline $\mathrm{x}$ & Axial or quantity for desired speed line \\
\hline
\end{tabular}




\section{Superseripts}

‘

$\mathrm{p}$

q

r

\section{Acronyms}

ANN

ASCII

CFD

EQL

HHV

LSE

PR

RMS

SF

SFC

SLC

SRE

TEIS

TIT

TZM

VTOL
Reference map condition

Relative flow condition

Mass flow rate extrapolation exponent

Specific work extrapolation exponent

Power extrapolation exponent
Artificial Neural Network

American Standard Code for Information Interchange

Computational Fluid Dynamics

Equilibrium Running Line

High Heating Value

Low Speed Extrapolation

Pressure Ratio

Root Mean Squared

Shape Factor

Specific Fuel Consumption (see Eq. 3.128)

Streamline Curvature

Simplified Radial Equilibrium

Two Elements In-Series

Turbine Inlet Temperature

Two-Zone Model

Vertical Take Off and Landing 


\section{Introduction}

Gas turbine technologies have been constantly evolving over the past fifty years and consequently the tools used for design have changed significantly to meet the new design requirements. Important goals at present are to maintain power output levels while maximizing efficiency and minimizing the weight and cost of the overall machine. Gas turbine engines are used in many applications, most notably being conventional, vertical take-off and landing (VTOL) aircraft and rotary-wing aircraft, marine propulsion and power generation. Gas turbine engines come in a wide variety of forms and configurations, including those with axial and radial compressor and turbine stages; the design choice usually depending on the performance specifications and application agreed upon at the outset of design. Furthermore, considerations into the combustion chamber, cooling methods, fuel delivery systems and ducting network are necessary while remaining within the performance specifications, structural integrity limits and cost structure of the project.

The focus of this thesis was the development of a tool for the synthesis of a centrifugal compressor map (also called compressor characteristics) from the limited information available at the preliminary design stage of a gas turbine engine. Knowledge of the compressor map at such early stages in the design process is unlikely unless altering a compressor of a previous design. Therefore, the ability to determine the compressor map accurately and rapidly early on in the design stage, allows for many 
design iterations to be completed and leads to the detailed design to be completed faster. Furthermore, this compressor map can be used to estimate the performance of the gas turbine engine for which it is being designed. The compressor map is a plot outlining the performance of the component over a wide variety of operating conditions. Figure 1.1 outlines a typical compressor map. The abscissa outlines the possible range of corrected mass flow rates of the compressor, while the y-axis indicates the values of pressure ratio. The solid dark lines on the compressor map indicate lines of constant rotational speed; for example, the solid dark line labelled 0.7 represents all the values of pressure ratio (as a function of corrected mass flow) for a rotational speed which is 70 percent of the design rotational speed. Furthermore, upon inspection of the compressor map, one is also able to notice the red contours overlaid on the pressure ratio characteristics. This is one particular way of indicating values of constant isentropic efficiency; for example the red contour labelled 0.81 corresponds to a contour where the isentropic efficiency of the compressor is 81 percent. 


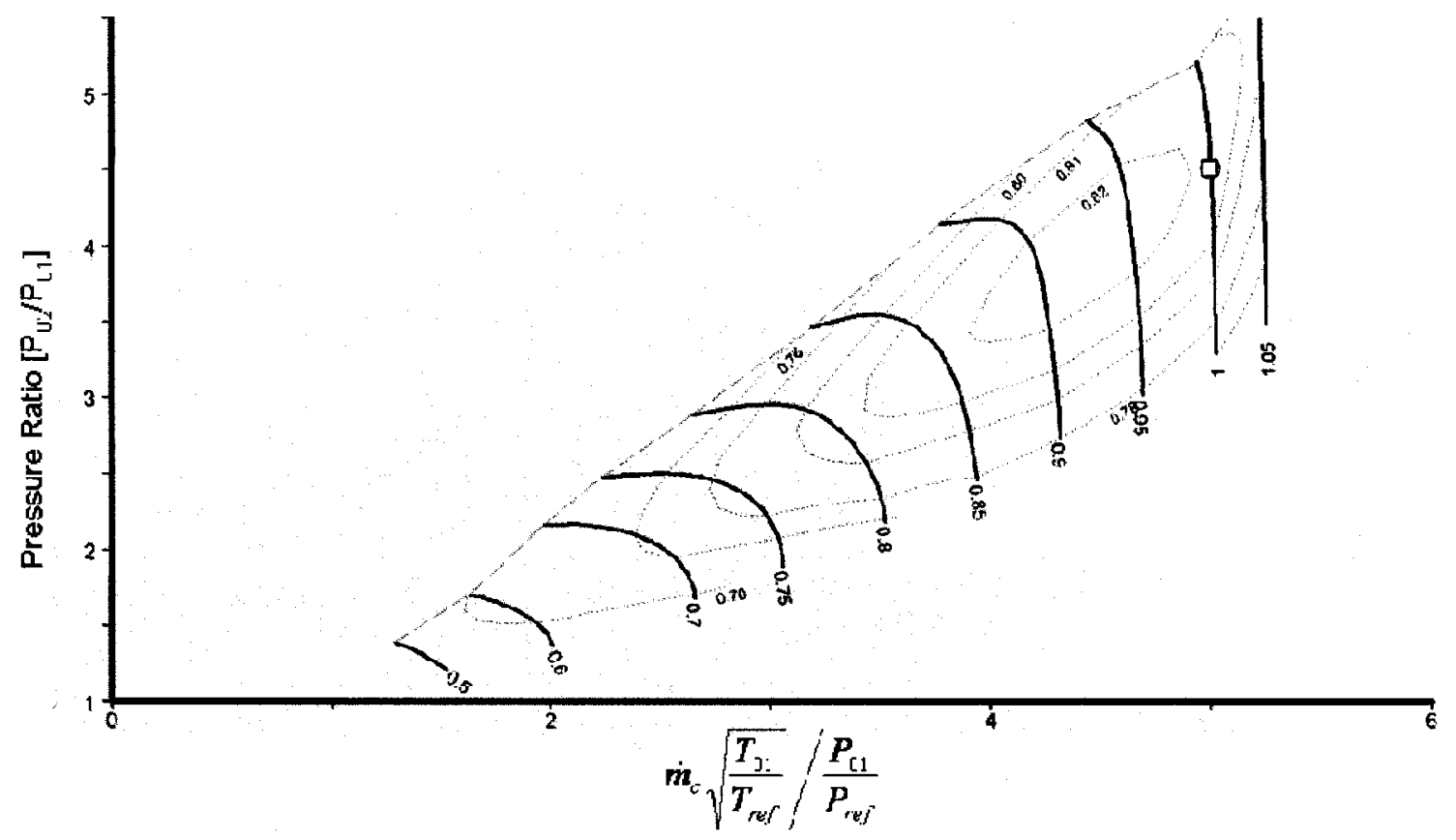

Figure 1.1 Typical compressor map.

Compressor corrected mass flow rate, compressor pressure ratio and compressor isentropic efficiency are all defined as follows:

- Compressor corrected mass flow rate: $\frac{\dot{m}_{c} \sqrt{\frac{T_{01}}{T_{r e f}}}}{\frac{P_{01}}{P_{r e f}}}$ where:

$\dot{m}_{c}$ denotes compressor air mass flow rate

$T_{0 I}$ denotes compressor inlet stagnation (or total) temperature $T_{r e f}$ denotes the reference temperature (usually $288 \mathrm{~K}$ )

$P_{01}$ denotes the compressor inlet stagnation (or total) pressure

$P_{\text {ref }}$ denotes the reference pressure (usually $101.3 \mathrm{kPa}$ ) 
- Compressor pressure ratio: $\frac{P_{02}}{P_{01}}$

where:

$P_{02}$ denotes compressor exit total pressure

$P_{01}$ denotes compressor inlet total pressure

- Compressor isentropic efficiency: $\eta_{c}=\frac{h_{02 s}-h_{01}}{h_{02}-h_{01}} \approx \frac{T_{02 s}-T_{01}}{T_{02}-T_{01}}$ where:

$\eta_{c}$ denotes compressor isentropic efficiency

$h_{02 s}$ denotes compressor exit ideal enthalpy

$h_{02}$ denotes compressor exit actual enthalpy

$h_{0 l}$ denotes compressor inlet actual enthalpy

$T_{02 s}$ denotes compressor exit ideal stagnation temperature

$T_{02}$ denotes compressor exit stagnation temperature

$T_{0 l}$ denotes compressor inlet stagnation temperature

The overall goal of the project was the design of a low-cost gas turbine engine for powerboat applications. The design was undertaken by a small group of graduate students in the Department of Mechanical and Aerospace Engineering at Carleton University. For the given application, several design choices were agreed upon. It was decided that the design would comprise a simple, single-spool turboshaft engine with a free power turbine. Figure 1.2 shows the overall engine configuration as decided by the research 
group design team. The engine consists of a single centrifugal compressor powered by an axial turbine. Engine output power is derived from the axial free power turbine. A numbering convention has been used to provide an indication of the location of flow within the gas turbine engine:

- Station 1: Atmospheric conditions

- Station 2: Compressor inlet

- Station 3: Compressor exit/Combustor inlet

- Station 4: Combustor exit/Gas generator turbine inlet

- Station 4.5: Gas generator turbine exit/Free power turbine inlet

- Station 5: Free power turbine exit

- Station 6: Exhaust duct

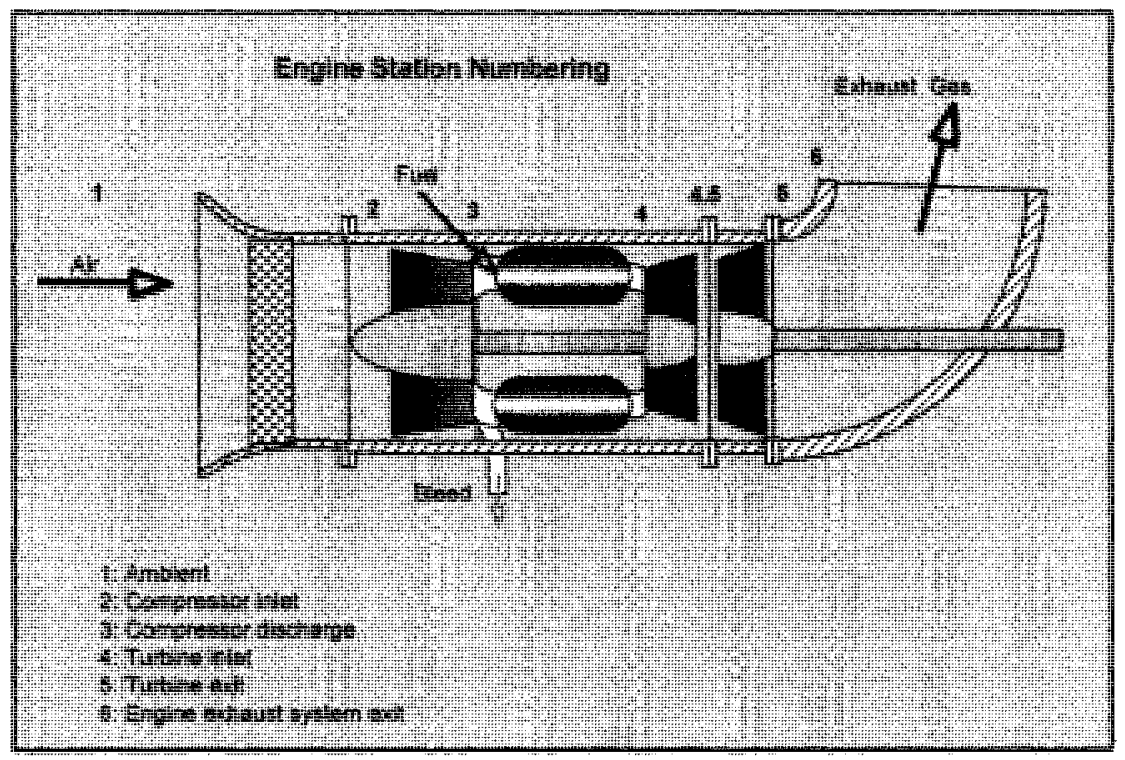

Figure 1.2 Gas turbine engine using a gas generator with a free power turbine.

Since this was the initial research initiative into determining overall engine performance within the design group, it was decided to simplify the analysis by performing the component matching with an engine performance without consideration 
of the free power turbine. Because the focus for the thesis was the compressor, a simple, single-spool turboshaft engine was employed for the engine performance modelling to simplify the implementation and testing of the developed algorithm. A schematic showing the single-spool turboshaft engine is shown in figure 1.3. Station numbering for the simple gas turbine unit is very similar to that of the gas turbine engine using a free power turbine, with the exception that station 4.5 is removed in the simple engine due to fact that there is only one turbine.

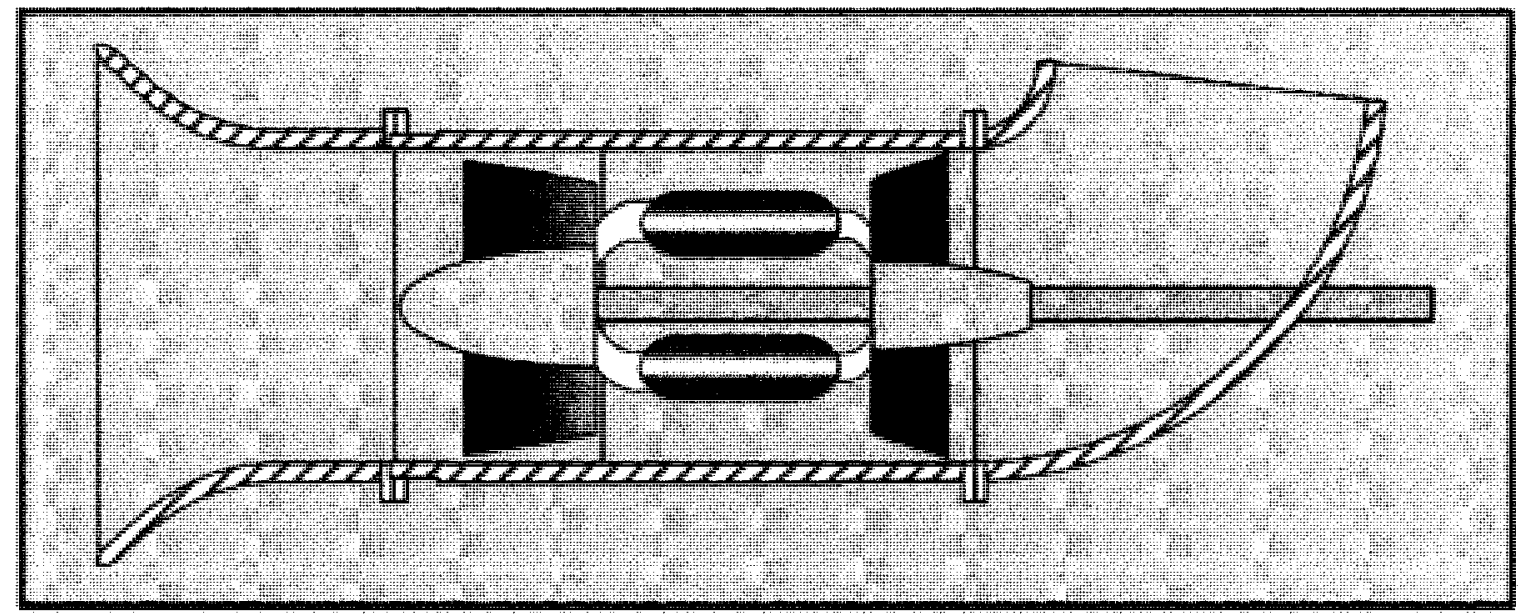

Figure 1.3 Simple, single-spool gas turbine engine schematic.

This thesis outlines the steps necessary to complete a computer code for the creation of a centrifugal flow compressor map and for the performance prediction of gas turbine engines. Given the preliminary design point information, the user of the computer code will be able to construct a full compressor map for use in engine off-design modelling using component matching calculations. 


\subsection{Overview}

Overall project guidelines stipulate the design of the centrifugal compression system, the combustion system, gas generation and free power turbine as well as the associated control system. Other members of the design team had the responsibility of each of the design components. An important phase of the design procedure however, was the estimation of the off-design behaviour of the engine once the components were designed. Such information is often required at an early stage of the design process to perform rapid iterations of the design.

The main problem associated with the off-design modelling process is the ability to determine or approximate to a reasonable degree of accuracy, the component maps for the compressor and turbine at an early stage of the design process. Once established, it is relatively simple to determine the behaviour at any operating point through "component matching" calculations. When gas turbine components such as the compressor and turbine are mechanically linked and constrained to run together, the range of possible operating points is severely limited by compatibility of mass flow through the engine, but also due to compatibility of rotational speed since the compressor and turbine are mechanically linked by a shaft (Saravanamutto, 2001). The problem then becomes finding those points where the engine is running at steady speed, which is termed equilibrium. These equilibrium points may be joined up on a compressor or turbine map to determine the equilibrium running line. The above steps are performed during the 
component matching calculations and furthermore require knowledge of both the compressor and the turbine performance maps. Several methods have been proposed and outlined in the literature for estimating the component maps and will be reviewed in subsequent sections. The applicability of these methods will also be discussed in those sections.

The main thesis goal is the development of a complete code to determine the offdesign performance of centrifugal flow compressors with the limited geometry data which would be available at the preliminary design stage. To achieve the goals for the off-design performance analysis three main tasks were decided upon at the outset of the project and include:

- $\quad$ Literature review outlining:

- Typical engine design cycle

- Compressor off-design performance analysis methods

- Importance of turbomachinery characteristics in engine performance modelling

- Component matching methods

- Performance estimation

- Development of the engine off-design analysis tool

- Validation, results and discussion 


\section{Literature Review}

The following chapter is a review of theory relating to the modelling of centrifugal compressors as well as the off-design modelling of gas turbine engines. Initially an overview of the typical design cycle will be outlined to give a scope of the use of the proposed design tool within the overall engine design phase. This will be followed by reasons for performing off-design analyses as well as the requirements for performing the off-design analysis. An introduction to the compressor map will be provided as well as a brief overview of the available options for centrifugal compressor performance prediction. This will be followed by a discussion of the turbine map and its use. Following this, component matching theory will be discussed along with the relevant performance parameters which are important to the engine and component designers.

This chapter only provides an overview of the techniques used for compressor modelling and engine off-design modelling. A more detailed discussion of any particular theory will be left for the section dealing with the development of the specific off-design analysis tool.

\subsection{Gas Turbine Design Procedure}

The typical design procedure for a given gas turbine is iterative with each new design procedure requiring input from other aspect of the design cycle and a flowchart of 
this cycle is shown in Figure 2.1. A flowchart describing the use of the off-design analysis tool is provided later. 


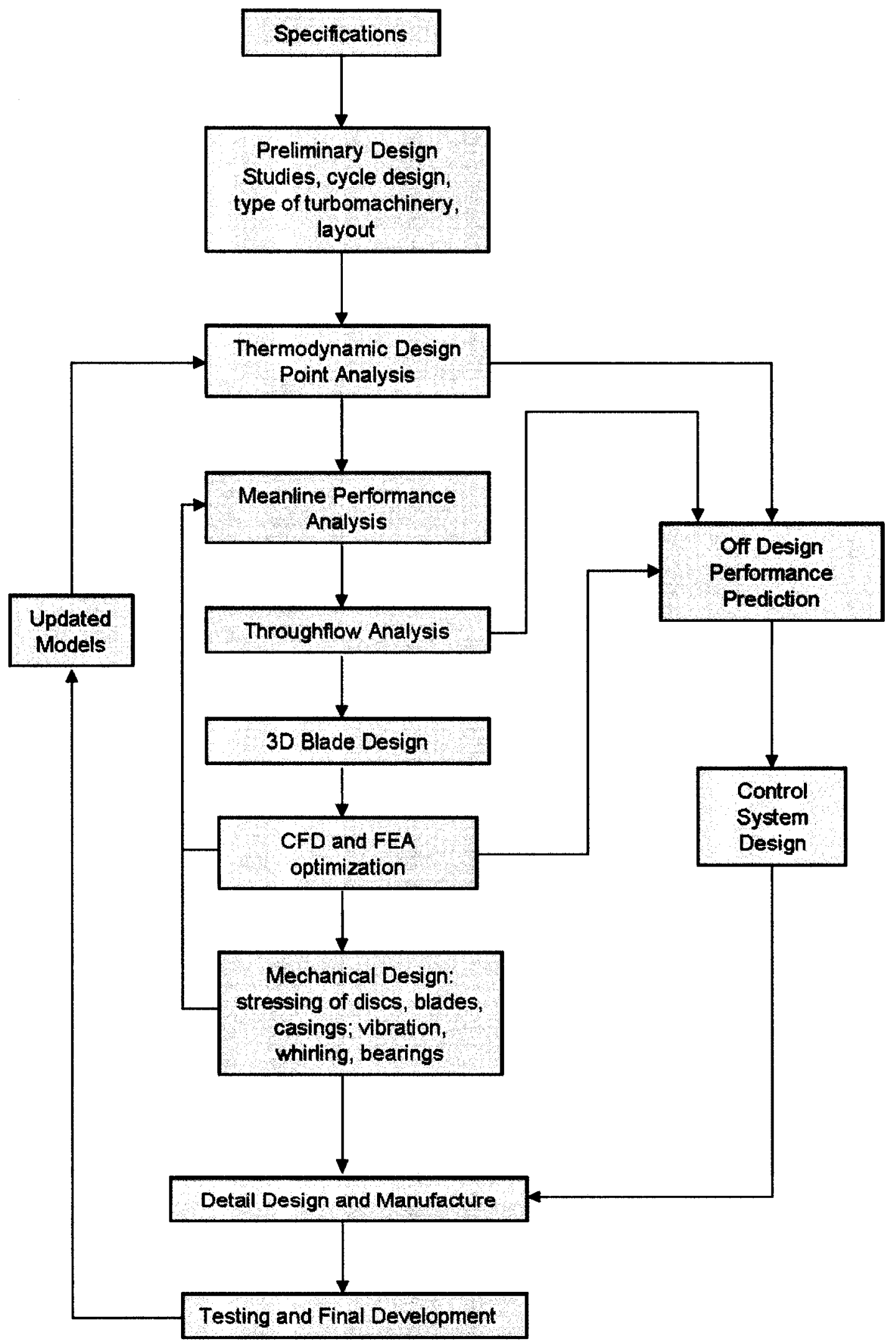

Figure 2.1 Typical turbomachinery component design procedure. Based on Saravanamuttoo et al. (2001). 
The design process shown in Figure 2.1 is highly iterative. The process usually begins with customer specifications which can be merely a requirement of power and efficiency. Factors such as weight, cost, fuel consumption, noise constraints and life play an important role early in the design phase. The initial major step is the thermodynamic design point determination where the turbine inlet temperature (TIT), pressure ratio (PR), air mass flow requirement and any air bleeds are calculated. This calculation can be based upon simple meanline calculation theory. Several textbooks exist outlining these calculations and the reader is referred to both Saravanamuttoo et al. (2001) and Dixon (1998) for a more detailed discussion of that theory. Subsequently, the aerodynamic design can be performed which is broken down into different steps. Initially, meanline calculations are performed to estimate the performance of the machine followed by a more thorough throughflow analysis. Meanline calculations refer to the determination of flow conditions at the location corresponding to the mean height of the blade within the blade row of the compressor or turbine. Throughflow analysis refers to a more complex calculation where the flow is considered at multiple points along the blade height, starting from the hub of the blade, extending to the tip. For axial machines, meanline and throughflow calculations are performed at each stage within the component whereas meanline and throughflow analysis for centrifugal machines are usually only performed at the inlet and exit of the impeller. Several methods for the throughflow analysis are available to the designer and a choice is made at an early stage as to which method will be used. Following these stages, a complex three-dimensional blade design is performed followed by a CFD analysis to estimate the performance. Furthermore, a finite element analysis is performed to determine the suitability of the stresses at the hub 
(Saravanamuttoo et al. (2001)). Once the engine has been designed, often its performance is re-investigated using newly updated and developed models in attempts to improve the overall engine design as much as possible.

While the above aerodynamic analysis is performed, an estimation of the engine off-design performance can be performed concurrently. Off-design refers to those conditions (e.g., rotational speed and mass flow rate) which the component is able to operate at, but was not designed to do. To determine the overall engine performance, estimations of the off-design performance of respective gas turbine engine components needs to be ascertained. There are several methods which can be used to estimate the component off-design performance: these will be outlined later. Engine performance modelling is a very important aspect of the overall design cycle. While the engine may run at the design point for the majority of operation, it is required to verify that the engine can operate safely and efficiently at other operating points as operating conditions can change rapidly during start-up, and as a result of atmospheric changes for aircraft engines.

Relating to the project at hand, different parts of the design cycle were taken into account by various members in the design group. For instance, certain members may be responsible for the aerodynamic and structural design of the turbine components while another may be developing a tool used for the aerodynamic design of the compression system. Furthermore, members may be developing a tool used to determine the combustion and control systems. It was the ultimate goal that these tools be integrated to 
be able to provide a complete detailed engine design program to be used for further designs within the student research group. Popular commercial software packages exist to determine component performance such as AxCent from Concepts NREC (Anon., 2007a) for the determination of both axial and radial compressor and turbine performance. Other software alternatives exist such as the popular cycle software package GasTurb ${ }^{\mathrm{TM}}$ (Anon. 2007b) which can be used for the estimation of overall engine performance. Both of the mentioned software packages are excellent at performing their respective functions: AxCent at determining performance characteristics for engine components and GasTurb ${ }^{\mathrm{TM}}$ at determining overall engine cycle performance, however both have their limitations as well. Both packages are quite expensive to obtain and furthermore AxCent does not perform gas turbine engine cycle performance while GasTurb ${ }^{\mathrm{TM}}$ does not have the ability to determine component maps. Furthermore, the engine off-design analysis of GasTurb ${ }^{\mathrm{TM}}$ is limited to the extrapolation of component maps from pre-existing maps to perform the component matching analysis, limiting the accuracy of the overall modelling.

\subsection{Gas Turbine Engine Off-Design Analysis}

It is worth noting at the outset, what the motivation is for performing off-design modelling. The question is begged, why undertake all the extra work if the engine will operate mainly at the design point? While this may be true for certain types of gas turbine engines, engine components will be required to operate at conditions far away from the design point including start-up, acceleration and deceleration as well as idling. Therefore, a requirement is to verify that the compressor will operate safely at all those conditions. 
Furthermore, uncontrollable factors such as ambient conditions can have a major influence on the performance of the engine.

Engine off-design analysis also allows for the determination of several important performance parameters including:

- Variation of turbine inlet temperature with rotational speed and mass flow rate

- Variation of net work output with rotational speed and mass flow rate

- Variation of specific fuel consumption with net work output

Determination of the above quantities allows the designers of the combustion and control systems to develop better models of the fuel schedules to provide the necessary gas temperature rises to meet the required performance.

To perform engine off-design analyses, component maps must be obtained, that is, a component map for each compressor and turbine in the engine. These maps are required to establish the equilibrium running line. The equilibrium running line, discussed previously, is the locus of points on a compressor map indicating the possible operating conditions of a gas turbine engine. As mentioned, when the compressor and turbine are mechanically linked, the possible range of operating points is drastically reduced due to the requirement of compatibility of flow through the engine (conservation of mass) as well as compatibility of rotational speed since the compressor and turbine are mechanically linked. Figure 2.4 shows and example locus of points overlaid on a compressor map indicating the equilibrium running line. 
Procurement of accurate compressor and turbine maps is often the biggest challenge when attempting to determine off-design performance. Both the compressor and turbine map show pressure ratio (for compressible flow machines) or head (incompressible flow machines) as a function of corrected mass flow and corrected rotational speed as well as the variation of isentropic efficiency with rotational speed and mass flow rate. Figures 2.2 and 2.3 show a typical compressor and turbine map, respectively.

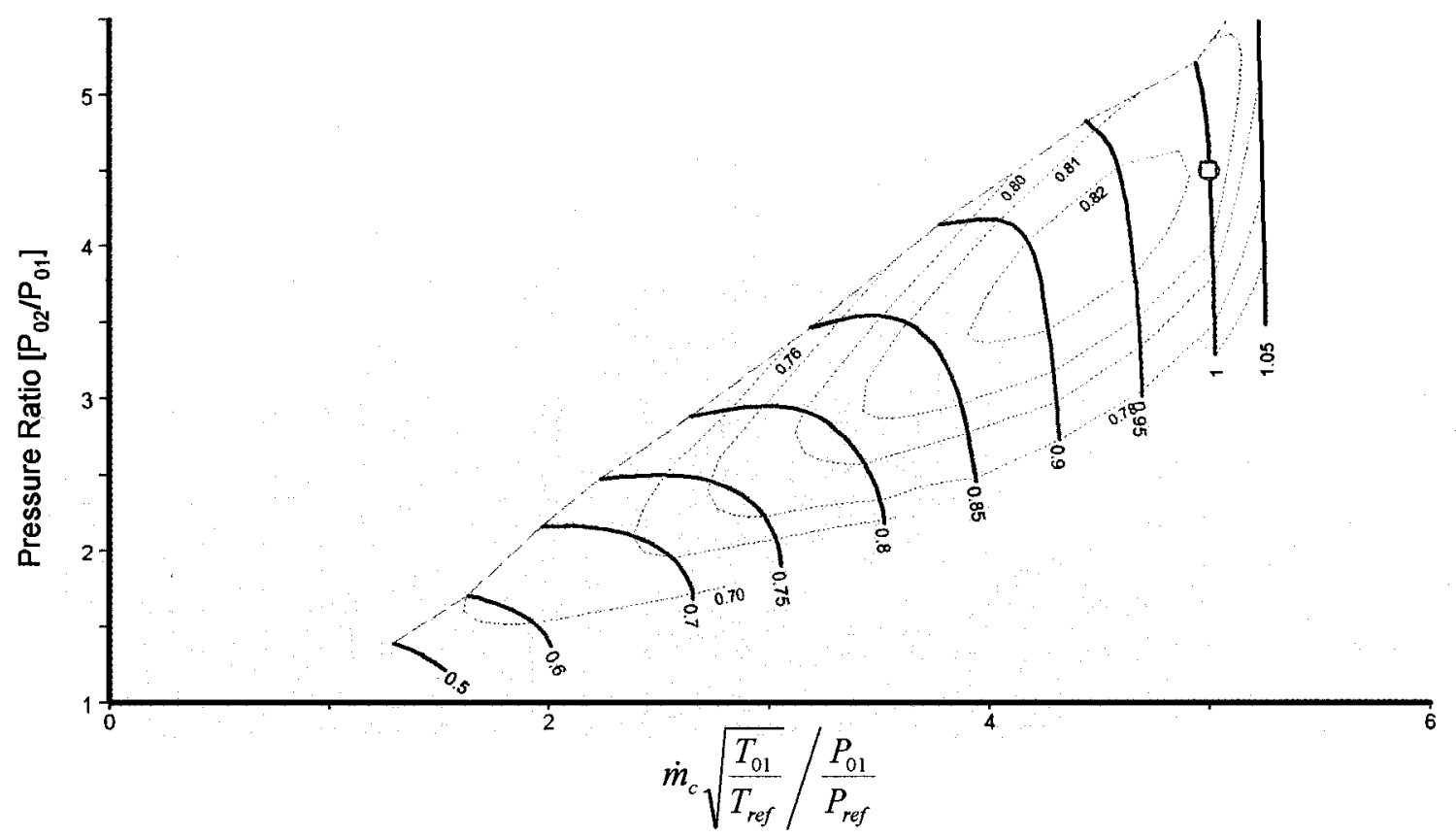

Figure 2.2 Typical compressor performance map. Obtained with the aid of GasTurb ${ }^{\mathrm{TM}}$. 


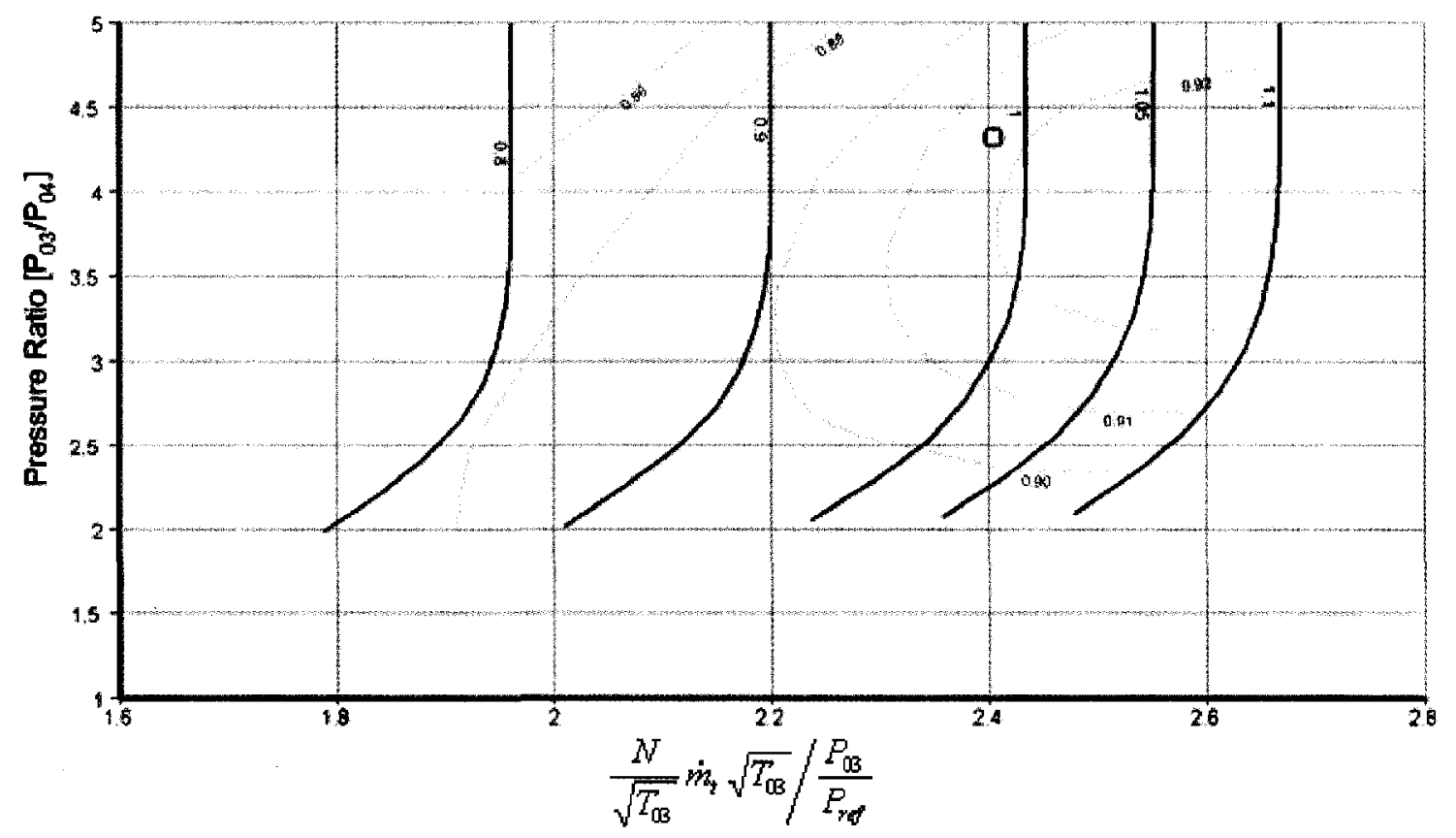

Figure 2.3 Typical turbine performance map. Obtained with the aid of GasTurb ${ }^{\mathrm{TM}}$.

Figures 2.2 and 2.3 show similar information for both a compressor and a turbine. Both are plots of corrected mass flow rate and pressure ratio for lines of constant speed. It should be noted that corrected mass flow in these two figures is defined slightly differently. Independent and dependant variables in these plots are defined as:

- Compressor corrected mass flow: $\frac{\dot{m}_{c} \sqrt{\frac{T_{01}}{T_{r e f}}}}{\frac{P_{01}}{P_{\text {ref }}}}$

- Compressor pressure ratio: $\frac{P_{02}}{P_{01}}$

- Turbine corrected mass flow: $\frac{N}{\sqrt{T_{03}}} \dot{m}_{t} \sqrt{T_{03}} / \frac{P_{03}}{P_{r e f}}$ where:

$N$ denotes the rotational speed in RPM 
$T_{03}$ denotes the turbine inlet temperature

$\dot{m}_{t}$ denotes the turbine air mass flow rate

$P_{03}$ denotes the turbine inlet stagnation pressure

$P_{\text {ref }}$ denotes the reference pressure (usually $101.3 \mathrm{kPa}$ )

- Turbine pressure ratio: $\frac{P_{03}}{P_{04}}$

where:

$P_{03}$ denotes the turbine inlet stagnation pressure

$P_{04}$ denotes the turbine outlet stagnation pressure

- Compressor isentropic efficiency: $\eta_{c}=\frac{h_{02 s}-h_{01}}{h_{02}-h_{01}} \approx \frac{T_{02 s}-T_{01}}{T_{02}-T_{01}}$

- Turbine isentropic efficiency: $\eta_{t}=\frac{h_{03}-h_{04}}{h_{03}-h_{04 s}} \approx \frac{T_{03}-T_{04}}{T_{03}-T_{04 s}}$ where:

$\eta_{t}$ denotes the turbine isentropic efficiency

$h_{03}$ denotes turbine inlet actual enthalpy

$h_{04}$ denotes turbine outlet actual enthalpy

$h_{04 s}$ denotes turbine outlet ideal enthalpy

$T_{03}$ denotes turbine inlet stagnation temperature

$T_{04}$ denotes turbine outlet actual stagnation temperature

$T_{04 s}$ denotes the turbine out ideal stagnation temperature 
$N$ refers to rotational speed of the engine, a quantity which is common to the compressor and turbine for components which are mechanically linked.

As in Figure 1.1, solid dark lines represent the characteristics for a single speed, for instance, the dark line marked 0.9 in Figure 2.2 corresponds to the characteristic when the compressor is operated at 90 percent of the design speed. Furthermore, the line marked 1.1 in Figure 2.3 corresponds to the turbine characteristic when the turbine is operated at a rotational speed which is 110 percent of the design speed. The light red lines shown in both figures are contours of constant isentropic efficiency for the respective component. For instance the value of 0.91 corresponding to the red contour in Figure 2.3 corresponds to those operating conditions where the isentropic efficiency of the turbine is 91 percent. Efficiency characteristics can be shown in this fashion, or can be provided as a separate plot which will be shown in subsequent sections.

Figure 2.4 shows an example of the equilibrium running line on a compressor map as was introduced in previous sections. This equilibrium running line was calculated by the GasTurb ${ }^{\mathrm{TM}}$ software package. The running line in Figure 2.4 can be estimated from component matching calculations, as outlined in sections 2.5 and 3.6. 


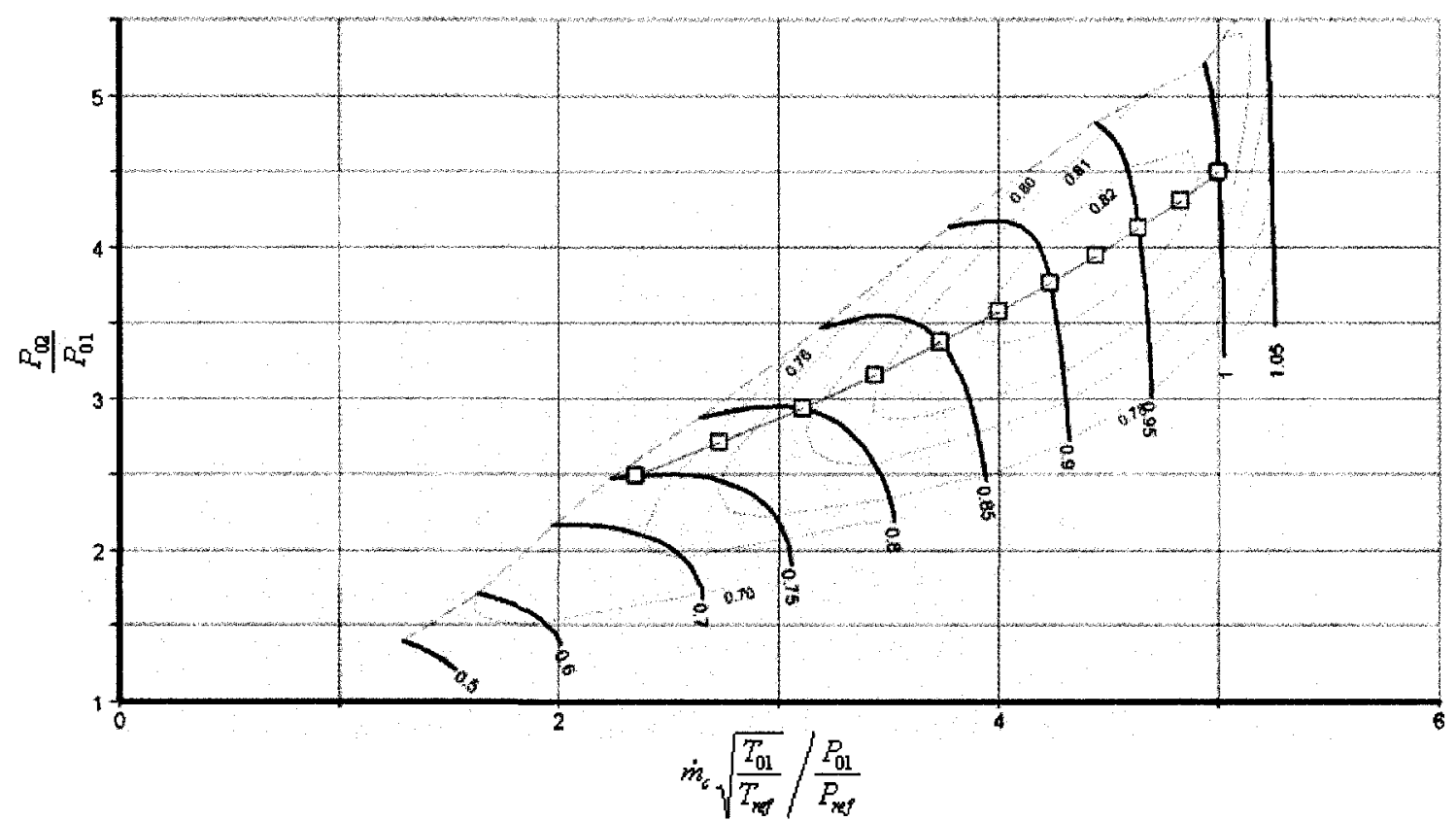

Figure 2.4 Equilibrium running line for a typical compressor. Obtained with the aid of GasTurb ${ }^{\mathrm{TM}}$.

Through the determination of the equilibrium running line, a designer is able to determine if the engine is operating within proper constraints, as well as the variation of efficiency as the engine moves towards the design point. The equilibrium running line furthermore gives an indication of the stability of the engine by determining the proximity of the operating line to the surge line. The surge line is indicated in Figures 2.2 and 2.4. It can be recognized as the dashed red line connecting the most left hand point of each of the compressor speed characteristics. The surge line is an important feature to note on the compressor map as it defines the point of unstable operation of the component. This unstable operation is usually due to a breakdown in the flow within the compressor or the diffuser (Saravanamutto et al., 2001). Knowing the proximity of the running line to the surge lines allows the engineer to determine the relative stability of the engine. Therefore, determination of the running line is a necessary step in calculating the part-load performance of any gas turbine engine. 


\subsection{Compressor Map Generation}

The generation of a compressor map is one of the most difficult tasks for the proposed project. Once a map has been established, the theory to determine the equilibrium running line and the determination of performance parameters is very well documented. However, the map determination still remains a significant roadblock in the overall algorithm.

Several methods exist for the determination of the compressor map which range in accuracy, ease of implementation and processing time. The most complex methods such as modelling in a computational fluid dynamics (CFD) software package or streamline curvature codes are often the most accurate. However, these methods require a large amount of designer experience and time to operate, let alone develop. Conversely, meanline solutions are often fairly straightforward in their implementation and require very little user input apart from the basic compressor geometry. However, the empirical correlations used for loss estimation are limited in their accuracy at conditions away from the design point. As mentioned previously, for preliminary design an approximation to the conditions is often adequate. Therefore, for a computer code to be useful for preliminary performance prediction, it will have the following qualities:

- Rapid computation time

- Minimal user input required

- Minimum amount of compressor geometry required for convergence

- Ability to handle a wide variety of flow conditions

- Accuracy while maintaining simplicity 
Meanline analyses are usually based upon a finite control volume which yield predictions of the mass-averaged outlet conditions, without modelling any of the throughflow gas path. These are often sufficient in their accuracy for use in preliminary design.

Often, the requirements for a performance prediction code have competing factors, for instance high accuracy and rapid computation time. It is the goal of this project to maintain the accuracy of the design tool while allowing for short computation times. This, in turn, will accommodate the large number of design iterations which are needed during the preliminary design phase. Furthermore, the selected impeller model will need to be integrated with a diffuser model to complete the performance prediction. Diffusers are components of a centrifugal compressor design used to recover static pressure by means of decelerating the high velocity flow at the impeller exit. There are two main types of diffusers used in conjunction with centrifugal impellers: vaneless and vaned diffusers. Vaneless diffusers simply consist of a vaneless space that increases the flow area between the diffuser inlet and exit. The increase in flow area leads to a decrease in flow velocity through conservation of mass. The other main type of diffuser is the vaned diffuser which consists of a number of stationary blades (also called vanes) which guide the flow through channels increasing in area from inlet to exit. The principle of pressure recovery between the two diffusers are identical, however a similar level of pressure recovery can be achieved in a much smaller space by means of a vaned diffuser leading to a smaller engine size (Aungier, 2000). For simplicity, a vaneless diffuser 
model was selected to complete the calculations; the addition of a vaned diffuser model is proposed for future versions of the performance prediction code.

Several methods exist for the determination of the compressor map, all of varying accuracy and complexity, including:

- Streamline curvature methods

- Map scaling techniques

- Stage stacking methods

- Row-by-row analysis

- Two-zone models

- One-Dimensional Loss Models

\subsubsection{Streamline Curvature Methods}

Streamline curvature (SLC) methods are possibly the most accurate methods outside of a CFD analysis of the impeller and are described in great detail by Boyer (2001). A stepwise methodology of solving flow conditions inside the blade passage is presented. Due to the iteration scheme and the complexity of the solving equations involved, streamline curvature methods are difficult to implement. Both the type of compressor and the flows within the compressor itself can compound the complexity of streamline curvature methods introducing instability into the equations. According to Daneshyar and Shaalan (1971), the streamline curvature process tends to overpredict changes in the radius of curvature of the estimated streamlines, a problem which would 
be compounded when attempting to determine the streamline shapes through the $90^{\circ}$ bend of a centrifugal compressor.

Conceptually, using SLC methods, the compressor is divided into a large number of adjacent stations as shown in Figure 2.5, known as quasi-orthogonals, which are usually located at, or between blade rows (never within an actual blade).

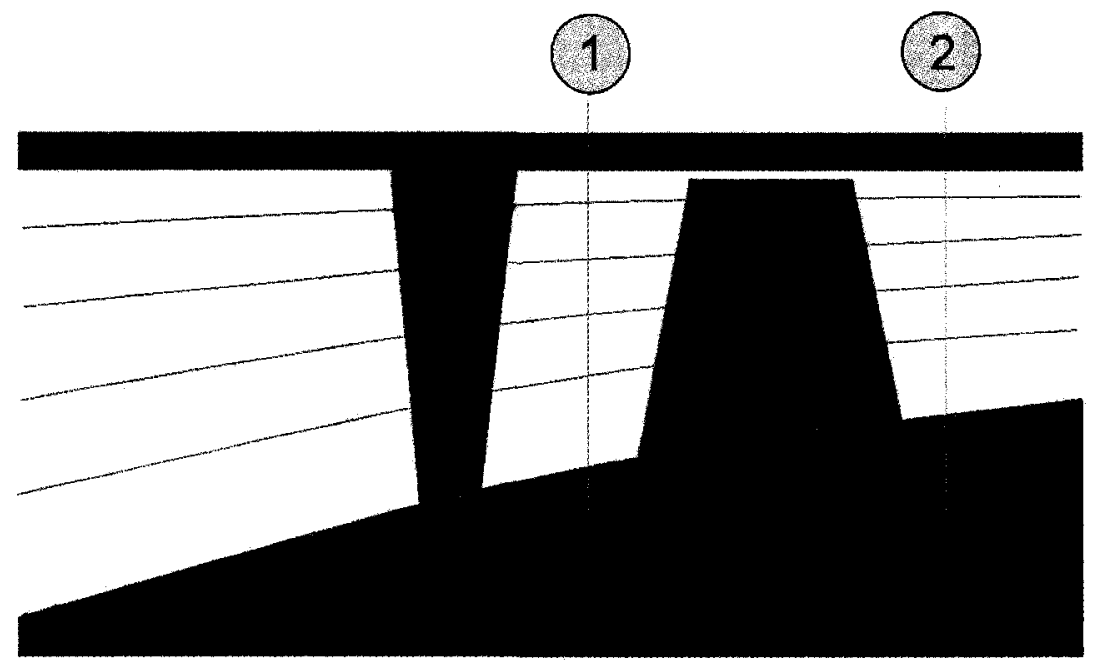

Figure 2.5 Computational domain for the streamline curvature method.

Gradients in both the meridional direction and spanwise direction are required for the streamline curvature analyses which are usually provided by external loss models and boundary layer development models. For a mathematical description of the streamline curvature design process, the reader is referred to the Ph.D. thesis by Boyer (2001). The general process for the streamline curvature analysis is summarized as follows:

1. The position of the streamline is guessed. This can be accomplished by either dividing the annulus into regions of equal mass flow for an assumed 
inlet velocity, or the annulus can be divided into equal radial distance with an uneven mass flow distribution between streamtubes.

2. The streamline curvature is then determined at the intersection of each quasi-orthogonal and streamline.

3. Guesses for the meridional velocity are supplied at the intersection of each quasi-orthogonal and streamline. Inlet stagnation properties are also supplied.

4. Cascade correlations are used to determine the flow angles at the exit of the rotor or stator.

5. The stagnation temperatures and pressure need to be estimated to determine the value of an entropy function. The absolute tangential velocity at the exit of a blade row can be determined by equations relating to the streamline curvature model. Stagnation properties are calculated and the spanwise meridional velocity distribution is determined.

6. The mass flow is determined across the annulus area. The mass flow is then checked against the initial value provided by the user. If the values match, continuity has been established. If the mass flow values do not match, the initially guessed meridional velocity is altered and the calculations continue until continuity has been established.

7. New values of the meridional velocity distribution will enable new estimations of the streamline positions to be established at each computing station. 
8. New positions of the streamlines are most likely not continuous; the solution tends to diverge at this point. A damping or relaxation factor is required to reduce the susceptibility to solution divergence.

9. Once new positions have been obtained for the streamlines at all computing stations, new streamline values can be established and Steps 5 through 9 are repeated until the movement of streamline positions is within a prescribed tolerance.

Despite the high degree of accuracy, streamline curvature algorithms are difficult to program due to their inherent complexity, but furthermore, are unsuitable for centrifugal machines. A coordinate frame transformation is required for centrifugal machines since the streamline curvature process operates accurately with small changes in streamline angle. Furthermore, the use of streamline curvature methods requires the complete compressor design to be provided, including the coordinates within the actual blade passages. Such information would not be available during the preliminary design phase and as such, the streamline curvature model is inapplicable to the project at hand.

Finally, as with all semi-empirical methods, results that are obtained by streamline curvature are highly dependent on the validity of the internal loss models accounting for profile losses, secondary losses and tip leakage. Improvement of the correlations used for these sections can vastly improve the results obtained (Pachedis et. al, 2006). Several commercial software packages utilize streamline curvature methods 
including AxCent from Concepts NREC (Anon. 2007a) and TEACC developed by Hale et al. (1994).

\subsubsection{Map Scaling Techniques}

Scaling methods have been popular for the determination of compressor characteristics. These techniques involve scaling an existing map to match the design pressure ratio and mass flow rate. This is the procedure which is performed within the $\operatorname{GasTurb}^{\mathrm{TM}}$ software. It is widely acknowledged that, while scaling techniques are accurate at conditions close to the design point, there is not much agreement with real engine conditions at off-design conditions (Kong et al., 2003).

Kurzke (2000) and Kong et al. (2003) both describe methods used for scaling component maps. Typically, maps are normalized to a common reference point. Corrected mass flow is normalized with respect to the design point corrected mass flow, pressure ratio is normalized with respect to the design point pressure ratio and rotational speed is normalized with respect to the design speed. Figure 2.6 is an example of a sample normalized compressor map obtained from Gauthier (2006). 


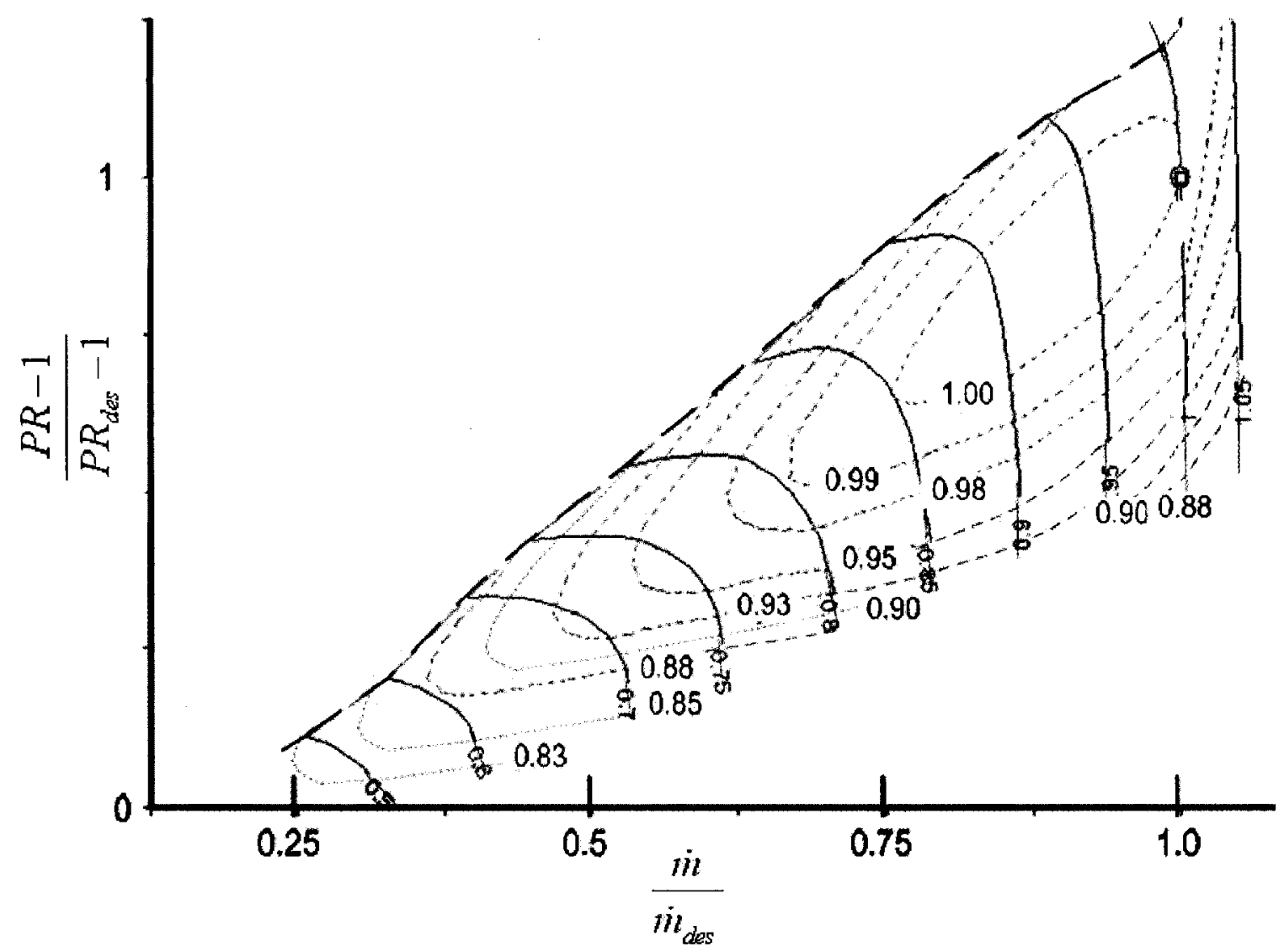

Figure 2.6 Sample scaled pressure ratio characteristic for use in the compressor map scaling procedure.

Once the map has been normalized, new map values for pressure ratio, corrected mass flow and isentropic efficiency can be calculated by:

$$
\begin{aligned}
& P R_{\text {new }}=\frac{P R_{\text {des }}}{P R_{\text {des org }_{\text {org }}}}\left(P R_{\text {scale }}-1\right)+1 \\
& \dot{m}_{\text {new }}=\frac{\dot{m}_{\text {des }}}{\dot{m}_{\text {des }}} \dot{m}_{\text {scale }} \\
& \eta=\frac{\eta_{\text {des }}}{\eta_{\text {des }_{\text {ori }}}} \eta_{\text {scale }}
\end{aligned}
$$

where: 
$P R_{\text {new }}$ denotes the new, scaled map pressure ratio

$P R_{\text {des }}$ denotes the new, scaled map design point pressure ratio

$P R_{\text {des }_{\text {org }}}$ denotes the original normalized map design point pressure ratio (usually unity)

$P R_{\text {scale }}$ denotes any, arbitrary original normalized map value

$\dot{m}_{\text {new }}$ denotes the new, scaled map mass flow rate

$\dot{m}_{d e s}$ denotes the new, scaled map design point mass flow rate

$\dot{m}_{\text {des }_{\text {org }}}$ denotes the original normalized map design point mass flow rate (usually unity)

$\dot{m}_{\text {scale }}$ denotes any arbitrary original normalized map value

$\eta_{\text {new }}$ denotes the new, scaled map isentropic efficiency

$\eta_{d e s}$ denotes the new, scaled map design point isentropic efficiency

$\eta_{\text {des }_{\text {org }}}$ denotes the original normalized map design point isentropic efficiency

$\eta_{\text {scale }}$ denotes any arbitrary original normalized efficiency value

For further explanation of the scaling procedure, the reader is directed to Kong et al. (2003) who give a more detailed derivation and also a comparison between different scaling methods. The method presented works well if the reference map is relatively similar to that for the new component. However any significant differences between the components severely limits the applicability of the scaling procedure. 
The above equations outline the most common and simplest scaling method. However, newer scaling methods have been proposed to improve the accuracy of the map at conditions further away from the design point. These newer scaling models operate similarly, however more quantities are required for scaling according to Kurzke (2000) or extra information at the off-design conditions are required according to Kong et al. (2003). Both of these factors contribute to the weaknesses associated with using such scaling procedures.

\subsubsection{Stage Stacking Methods}

Spina (2002) proposed a new version of the traditional stage stacking technique which is used commonly in industry and can be applied to both centrifugal and axial compressors. The method involves using a generalized compressor (or turbine) characteristic to determine new compressor characteristics as a function of flow and stage load coefficients, as well as empirical shape factors. Muir (1988) presented a generalized axial compressor curve (shown in Figure 2.7) which has been used successfully in many stage stacking methods. 


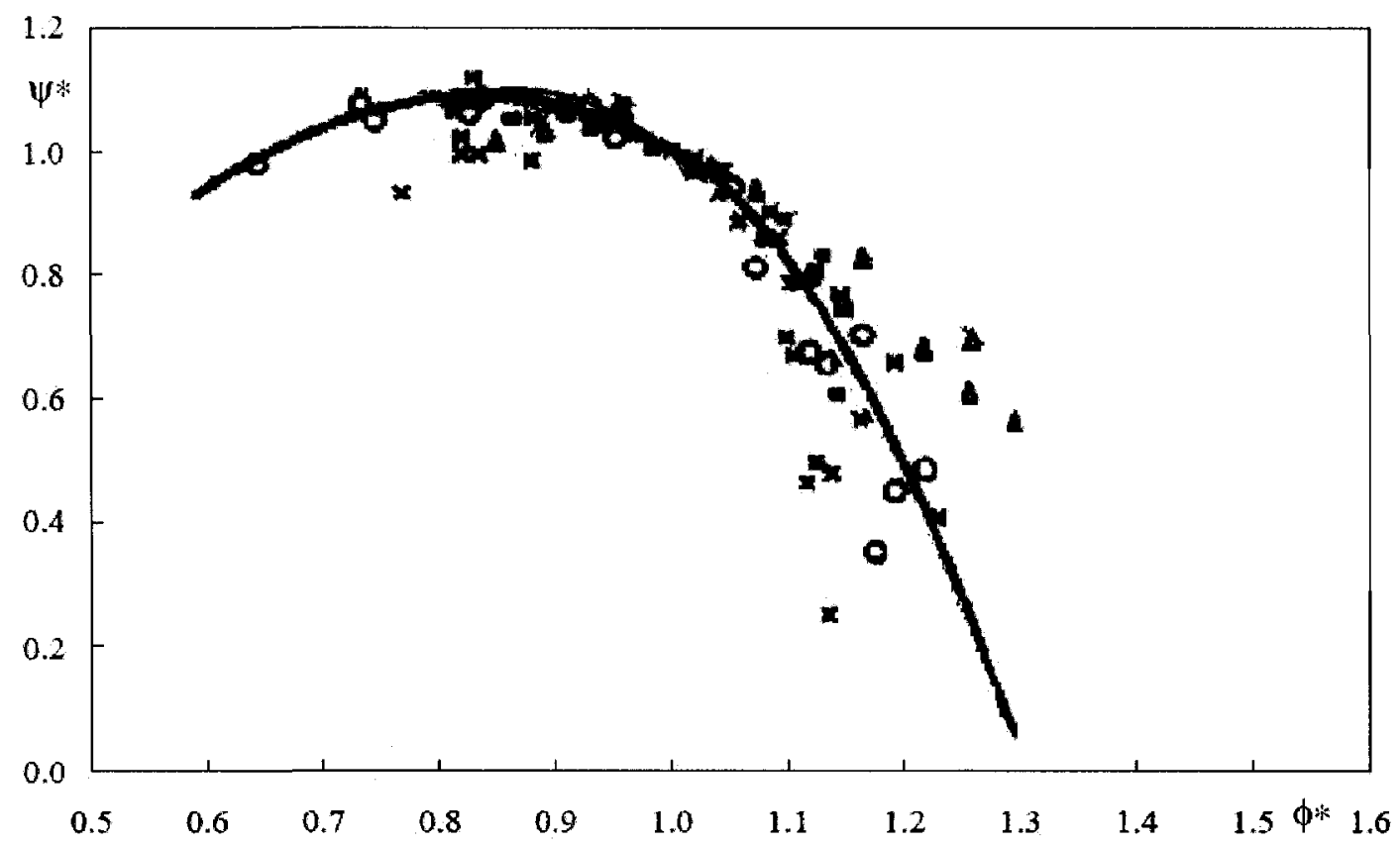

Figure 2.7 Generic axial stage compressor characteristic used in the stage stacking method..

Starting with the compressor inlet conditions, it is possible to determine the stage outlet conditions using the generalized curve once a reference condition has been selected (usually the design point). The process proceeds with the calculation of a nondimensional flow coefficient:

$$
\phi^{*}=\frac{\phi}{\phi_{r e f}}
$$

where:

$$
\begin{aligned}
& \phi^{*} \text { : Generalized stage flow coefficient } \\
& \phi: \text { Operating point flow coefficient } \\
& \phi_{r e f}: \text { Reference point flow coefficient (usually design point flow coefficient) }
\end{aligned}
$$


The flow coefficient is a dimensionless number used to relate flow conditions to the mass flow rate through the engine, defined as:

$$
\phi=\frac{C_{x}}{U}
$$

where:

$$
\begin{aligned}
& C_{x}: \text { stage axial velocity } \\
& U: \text { meanline blade speed }
\end{aligned}
$$

Once a value for the non-dimensional flow coefficient is determined, a value can be read from the generalized stage characteristic for the normalized stage aerodynamic load coefficient, $\psi^{*}$. Using this value of non-dimensional pressure coefficient, the actual stage load coefficient can be determined:

$$
\psi=\psi^{*} \psi_{\text {ref }}
$$

The stage aerodynamic load coefficient is a dimensionless number used to estimate the enthalpy increase through the stage, defined as:

$$
\psi=\frac{\Delta h_{0}}{U^{2}}
$$

where:

$\Delta h_{0}$ denotes the stage enthalpy rise

This value for stage load coefficient of the stage is then used in the following equations. With the inlet conditions are specified, the analysis proceeds in row-by-row fashion: 


$$
\begin{aligned}
& h\left(T_{0(i+1) s}\right)=h\left(T_{0 i}\right)+U_{i}^{2} \psi_{i} \\
& h\left(T_{0(i+1)}\right)=h\left(T_{0 i}\right)+\frac{U_{i}^{2} \psi_{i}}{\eta_{i}} \\
& \frac{P_{0(i+1)}}{P_{0 i}}=e^{\left[\Phi\left(T_{0(i+1) s}\right)-\Phi\left(T_{0 i}\right)\right]} \\
& \phi_{i+1}=\phi_{i} \frac{U_{i}}{U_{i+1}} \frac{A_{i}}{A_{i+1}} \frac{P_{0 i}}{P_{0(i+1)}} \frac{T_{0(i+1)}}{T_{0 i}}
\end{aligned}
$$

where:

$$
\begin{aligned}
& h(T): \text { Enthalpy } \\
& U: \text { Blade speed } \\
& \eta: \text { Isentropic efficiency }
\end{aligned}
$$

$A$ : Flow area

$\Phi$ : Entropy function $\Phi=\frac{1}{R} \int_{T_{r e f}}^{T} c_{p}(T) \frac{d T}{T}+\Phi\left(T_{r e f}\right)$

$i$ : Stage inlet condition

$i+1:$ Stage exit condition

This fashion proceeds with the exit conditions of the first stage becoming the inlet conditions of the second stage, and so on. With the conditions determined across $n$ stages, it is possible to evaluate the stage pressure ratio at the given flow coefficient by: 


$$
\begin{aligned}
& P R=\frac{P_{0(n+1)}}{P_{01}} \\
& \Delta h_{0}=h\left(T_{0(n+1)}\right)-h\left(T_{01}\right) \\
& h_{0(n+1) s}=c_{p}(\bar{T})\left(T_{01}(P R)^{\frac{\gamma}{\gamma-1}}\right) \\
& \eta=\frac{h_{0(n+1) s}-h_{01}}{\Delta h_{0}}
\end{aligned}
$$

where,

$$
\begin{aligned}
& T_{0(n+1)} \text { : Compressor exit stagnation temperature } \\
& \bar{T}=\frac{T_{01}+T_{0(n+1)}}{2} \text { : Estimate of mean temperature } \\
& \eta: \text { Isentropic efficiency } \\
& n+1: \text { Compressor exit location }
\end{aligned}
$$

Since the centrifugal machine is only one stage, the calculation procedure is highly simplified. However, it requires the knowledge of the centrifugal stage characteristics. Furthermore, since the derived component map is based upon a generalized curve, it is susceptible to the same errors as the scaling technique. Using a generalized curve fails to capture the flow physics specific to the compressor under the design process and, as with other methods, is highly dependent on the validity of the empirical correlations employed. 


\subsubsection{Row-by-Row Analysis}

Another method for the prediction of compressor maps is the row-by-row analysis. The row-by-row method is a one-dimensional method where the losses are accounted for a modified diffusion factor and are subsequently mass-averaged over the span of the blade. Flow physics are modelled at the blade row meanline position by the solution of the velocity diagram and employ modified forms of the load coefficient to determine the enthalpy changes across each blade row.

The row-by-row analysis method is a relatively simple technique which has the ability to produce accurate results for a wide variety of operating conditions, design speeds and design pressure ratios. The reader is directed to Attia and Schobeiri (1995) who describe a full set of equations for the estimation of axial compressor maps from simple design point geometry and design point operating conditions. The model itself appears to be highly applicable to axial compressors. However, the correlations for the total loss parameter as a function of the modified diffuser factor have not been established for centrifugal compressors.

\subsubsection{Two-Zone Modelling}

The two-zone model is a technique developed over the years by several authors and has been rigorously investigated as a performance prediction tool since the $1960 \mathrm{~s}$. The two-zone model is strictly a model for predicting the performance of centrifugal compressors. Oh et al. (2002) provided a new version of the long used two-zone model. 
Originally based upon the work of Japikse (1985), the model splits the impeller flow into two regions: an isentropic jet and a loss containing wake, where the wake flow follows the blade exactly while the jet flow is able to deviate from the impeller wall thereby accounting for slip. Jet/wake flow phenomena have been observed by several individuals including Senoo et al. (1968), later confirmed in the work of Eckhardt (1975). Table 2.1 indicates the relative differences between the two two-zone models proposed by Japikse (1985) and Oh et al. (2002).

Table 2.1 Differences between two-zone model methods.

\begin{tabular}{|l|c|c|}
\hline & Japikse (1985, 1996) & Oh et al. (2002) \\
\hline Wake mass flow fraction & Fixed & Variable \\
\hline Slip & Constant & Variable \\
\hline Wake deviation from blade & None & Allowed \\
\hline Unloaded tip assumption & Employed & Employed \\
\hline
\end{tabular}

Wake mass flow fraction is defined as the ratio of the wake mass flow to the total mass flow through the impeller passage. Slip is the observed deviation from the impeller exit due to pressure variation within the blade passage. The Japikse model $(1985,1996)$ fixes these quantities while the Oh et al. model (2002) allows those quantities to vary, more accurately capturing the flow physics within the impeller.

Table 2.2 outlines the relative merits and drawbacks of the both the Japikse model $(1985,1996)$ and the Oh et al. model $(2002)$. 
Table 2.2 Benefits and drawbacks to two separate two-zone models.

\begin{tabular}{|l|l|l|l|}
\hline \multicolumn{2}{|c|}{ Japikse Model $(1985,1996)$} & \multicolumn{2}{c|}{ Oh et al. Model (2002) } \\
\hline $\begin{array}{l}\text { Benefits } \\
\text { implement }\end{array}$ & \multicolumn{1}{c|}{ Drawbacks } & Benefits & \multicolumn{1}{c|}{ Drawbacks } \\
\hline $\begin{array}{l}\text { Requires little } \\
\text { iteration }\end{array}$ & $\begin{array}{l}\text { Older loss models } \\
\text { employed }\end{array}$ & $\begin{array}{l}\text { Utilizes many } \\
\text { updated } \\
\text { correlations } \\
\text { implement }\end{array}$ & $\begin{array}{l}\text { Tendency to } \\
\text { diverge due to } \\
\text { wake mass flow } \\
\text { fraction correlation }\end{array}$ \\
\hline Converges well & More realistically & $\begin{array}{l}\text { More } \\
\text { captures flow } \\
\text { physics }\end{array}$ & \\
\hline
\end{tabular}

Table 2.2 indicates that while the Japikse $(1985,1996)$ model is simpler to implement, it is also less accurate, possibly in part due to the older external loss models employed. The Oh et al. (2002) model, more accurately captures flow physics within the impeller however is more difficult to implement due to the increased iteration required for implementation.

Depending on the exact set of equations used to describe the two-zone model, two or three assumptions are required: 1) impeller tip static pressure for the jet and wake flows are identical (unloaded tip conditions) resulting in no pressure gradient across the jet and wake, 2) mixing of the jet and wake flow occurs just downstream of the impeller exit and not in the impeller passage and 3) the wake flow fraction can be assumed to be either constant, lying somewhere between 0.15 and 0.25 (according to Japikse (1996)), or in more recent attempts to describe the two-zone model, the wake mass flow fraction is left variable and correlations are used to establish the correct value (according to Oh et al. (2002)). 
Figure 2.8 shows a representation of the jet-wake flow at the impeller exit, as used in the two-zone model.

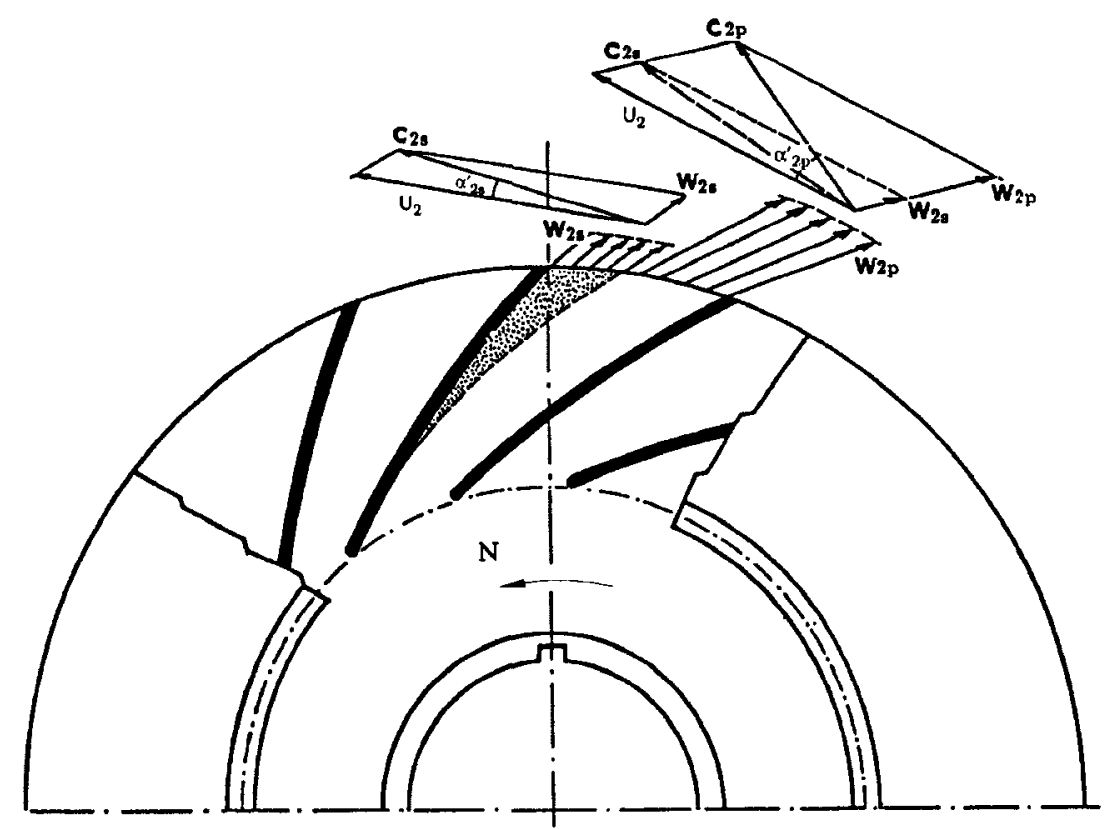

Figure 2.8 Schematic of the two-zone model impeller exit flow.

The two-zone model is useful as a preliminary design tool for establishing impeller maps as it requires minimal information of the impeller itself, namely the geometry which could be established by simple design point calculations. Required parameters for the two-zone model include:

- Inlet Stagnation Pressure $\left(\mathrm{P}_{01}\right)$

- Inlet Stagnation Temperature $\left(\mathrm{T}_{01}\right)$

- Design mass flow rate $(\dot{m})$

- Design speed $\left(\mathrm{N}_{\text {des }}\right)$

- Inducer hub radius $\left(\mathrm{r}_{1 \mathrm{~h}}\right)$

- Inducer tip radius $\left(\mathrm{r}_{1 \mathrm{t}}\right)$ 
- Outlet radius $\left(\mathrm{r}_{2}\right)$

- Blade height at inlet $\left(b_{1}\right)$

- Blade height at outlet $\left(b_{2}\right)$

- Backsweep angle $\left(\beta_{2 b l}\right)$

- $\quad$ Number of full blades $\left(Z_{\text {full }}\right)$

- $\quad$ Number of splitter blades $\left(Z_{\text {split }}\right)$

- Blade thickness at outlet $\left(\mathrm{t}_{2 \mathrm{~b}}\right)$

- Inlet hub blade angle $\left(\beta_{1 b l_{h}}\right)$

- Inlet tip blade angle $\left(\beta_{1 b l_{t}}\right)$

- Design pressure ratio $\left(\mathrm{PR}_{\mathrm{des}}\right)$

Furthermore, no internal loss correlations are required since the specification of the unloaded tip condition leads to a direct calculation of the entropy increase between the inlet and exit of the impeller. Despite this advantage, loss correlations are required for the external losses not accounted for inherently in the model, specifically recirculation loss, disc friction loss and tip leakage loss.

The reader is directed to several sources for explicit formation of the equations governing the two-zone model. Japikse $(1985,1996)$, Oh et al. (2002) and Whitfield (1990). All give various versions of the set of equations governing the two-zone model and the associated loss models. 


\subsubsection{One-Dimensional Loss Models}

The final method investigated for the performance prediction of centrifugal compressors was that of a traditional one-dimensional calculation using internal and external loss correlations to help describe the flow.

Many authors have described various sets of correlations to determine the losses associated with centrifugal compressors. However, all have the common goals of determining the velocity triangles at the inlet and exit of the impeller. Aungier (2000) and Dixon (1998) both describe a full set of equations with the aim of the determining impeller performance over a wide range of operating conditions.

Oh et al. (1997) describe a full set of equations for the performance prediction of centrifugal impellers. At the time of publication, the sets of equations described show the optimum combination of available loss model alternatives for the most accurate representation of centrifugal compressor performance. Accounted for in the analysis are internal loss models describing:

- Incidence loss

- Blade loading loss

- Skin friction loss

- Clearance loss

- Mixing loss

- Vaneless diffuser loss 
Furthermore, Oh et al. (1997) describe the optimum combination of external loss correlations. This is of significance since the external loss correlations could be used in association with other impeller performance prediction methods such as the two-zone model. The optimum combination of external loss models include mechanisms to account for:

- Disc friction loss

- Recirculation loss

- Tip leakage loss

The external losses outlined above are further discussed and defined in section 3.1.6. The set of loss models described by Oh et al. (1997) was used successfully by Roberts (2001) to predict the off-design behaviour of centrifugal compressors. 


\subsubsection{Compressor Map Generation Summary}

It is apparent from examination of the literature, that there is no "best" methodology for determining component maps. Each performance prediction model examined has its relative merits as well as shortcomings. Decisions were made regarding model accuracy, difficulty of implementation, degree of use in literature and computation requirements to select the best combination of impeller and diffuser models to develop the necessary algorithm.

\subsection{Turbine Map Generation}

A typical turbine map is shown in Figure 2.9 obtained with the aid of GasTurb ${ }^{\mathrm{TM}}$ (Anon., 2007b) however this map shown can be different from many types encountered in practice.

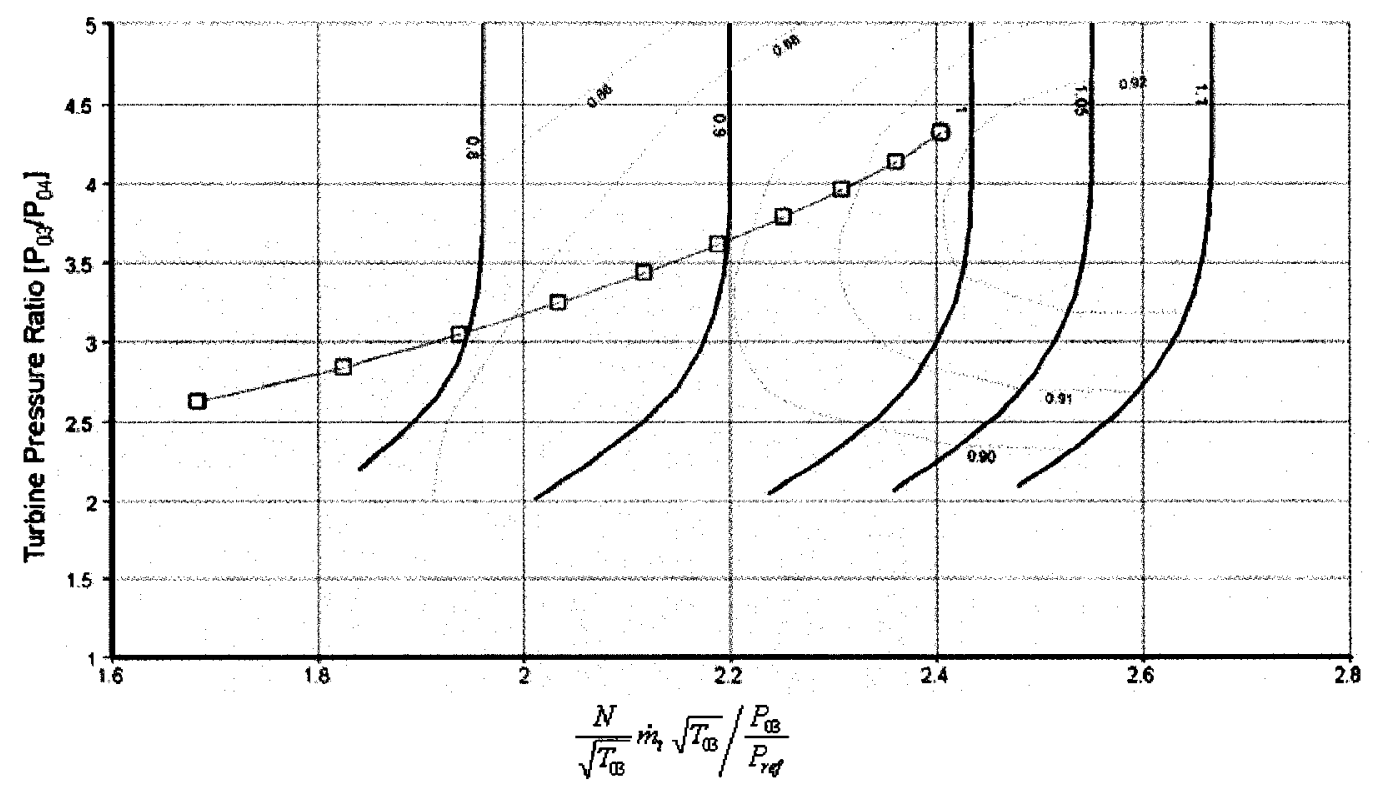

Figure 2.9 Sample turbine map showing the running line established by component matching calculations. 
Often, the parameters on the $\mathrm{x}$ and $\mathrm{y}$ axes are reversed; that is, pressure ratio or head rise on the $\mathrm{x}$-axis with the corrected mass flow on the y-axis. Since the turbine is directly downstream of the compressor and combustion systems, it typically operates at similar pressure ratio which is fixed by the pressure rise across the compressor. In essence, the pressure ratio becomes the independent variable and one is attempting to match mass flow to ensure continuity between the gas generator turbine and its respective compressor.

There are many ways turbine maps can be estimated and many of the generation techniques discussed previously apply to the turbine as well. Despite the need to obtain a turbine map to ascertain performance, according to Saravanamuttoo et al (2001), often the turbine operates at a fixed point and the final results of the component matching algorithm and performance estimation are not sensitive to turbine operating conditions. Therefore, one is able to use a single curve, or even a single point for use in the component matching calculations, greatly simplifying the analysis. Since the turbine map is less important than the compressor map for performance prediction due to its operation at, or about a fixed point, simple scaling techniques (as outlined in previous sections) are appropriate for determining the turbine characteristic. 


\subsection{Component Matching Techniques}

The previous sections have outlined various techniques to determine the overall components maps from their respective geometries to a reasonable degree of accuracy. These diagrams show the operational ranges which are typical during normal use and can be used to determine the performance of an individual component at a particular operating condition. However, when the components are linked together in an engine and are required to run harmoniously, the range of possible operating points is dramatically reduced. Such is the case in even the simple single-spool power unit. Furthermore, even a minor increase in the complexity of the engine as in this case of a gas generator with free power turbine severely limits the possible operating points of the engine, as will be seen subsequently. The main problem arises in the need to find corresponding operating points for each component characteristic such that the entire engine is running at steady speed or is in equilibrium. Subsequently, the equilibrium points may be established for a series of rotational speeds and may be joined up to form an equilibrium running line. Once the running line is determined, it is relatively simple to determine performance parameters such as specific fuel consumption (SFC) or power output as a function of flow rate. Figure 2.4 shows a typical equilibrium running line for an intermediate pressure ratio compressor.

The analysis considered for this particular project is the running of a single-spool gas turbine engine. However, methodologies exist for the determination of the running line when the gas turbine engine consists of a gas generator with a free power turbine, or a gas generator with a nozzle. Theory relating to the determination of the running line for 
a single shaft engine is outlined in subsequent chapters, however the reader is directed to Saravanamuttoo et al. (2001) for a comprehensive review of theory pertaining to more complex running line calculations.

Inherent in all methods of running line estimation, is the need to ensure compatibility of flow, compatibility of rotational speed and compatibility of work between the compressor and turbine, that is, one must ensure that the mass flow through compressor matches the flow turbine (less any bleeds), that the rotational speed of the compressor equals the rotational speed of the turbine (since they are mechanically linked by the same shaft) and that the turbine produces at the very least, the amount of power required to drive the compressor at that operating point. The power developed by the turbine and the power required by the compressor at any given operating point can be calculated as follows:

$$
\begin{aligned}
& \dot{W}_{t}=\dot{m} C_{p} T_{03} \eta_{t}\left[1-\left(\frac{1}{P R_{t}}\right)^{\frac{\gamma-1}{\gamma}}\right] \\
& \dot{W}_{c}=\frac{\dot{m} C_{p} T_{01}}{\eta_{c}}\left[P R_{c}^{\frac{\gamma-1}{\gamma}}-1\right] \\
& \dot{W}_{n e t}=\dot{W}_{t}-\dot{W}_{c}
\end{aligned}
$$

With the above theory, one is able to determine the net power developed at the design point, since the design mass flow rate, pressure ratio and efficiencies are generally 
known from simple design point calculations. Furthermore, determining the developed design point net power allows for the power requirement at part speed conditions. According to Saravanamuttoo et al. (2001), the net power output of the engine varies with the cube of the rotational speed:

$$
\begin{aligned}
& \dot{W}_{n e t} \propto N^{3} \\
& \dot{W}_{n e t} \approx k_{1} N^{3}
\end{aligned}
$$

Knowing the design point operation (pressure ratio, corrected mass flow rate and rotational speed) one is able to determine the power produced by the turbine and power required for the operation of the compressor. Rearrangement of Equation 2.20 allows for the constant $k_{I}$ to be calculated and further used to determine the net work output at other rotational speeds. Further component matching calculations can then be performed to determine the location on the speed characteristic where the power requirement is met. When the above analysis is performed for a series of speed lines, the equilibrium running line can be established. Specific equations and any required models for the component matching calculations can be found in subsequent sections.

\subsection{Summary}

A brief overview of the typical gas turbine engine design cycle has been presented as well as the location of off-design analysis within the overall framework. Furthermore, 
a synopsis of the problems and challenges associated with engine off-design analysis has been discussed.

An overview of the existing methods for the estimation of the performance of individual gas turbine engine components has also been presented. The chosen focus of the work was the estimation of the centrifugal compressor map, as overall engine performance is highly sensitive to the accuracy of these characteristics. In-depth description of the different models is not presented, merely the relative merits of each of the models and any concerns of implementation in a computer code. An overview of the component matching calculations is also presented. Using the materials reviewed, a design tool for the performance prediction of centrifugal flow compressors and their influence in gas turbine engine design is presented next. It is the hope of the author that such a tool can be useful for the performance prediction of centrifugal flow compressors and their role in gas turbine engine use, early on in the design phase with a reasonable degree of accuracy. 


\section{Proposed Centrifugal Compressor Map Synthesis Tool and Off-Design Engine Performance Prediction Tool}

The following chapter outlines the theory behind the selected models used to accurately describe the complete centrifugal compressor map and also determine gas turbine engine performance. Included in this, are the models to describe the impeller performance as a function of the geometry and flow conditions, vaneless diffuser performance, sub-idle extrapolation model and component matching tools. A flowchart of the overall design tool algorithm is shown in Figure 3.1. 


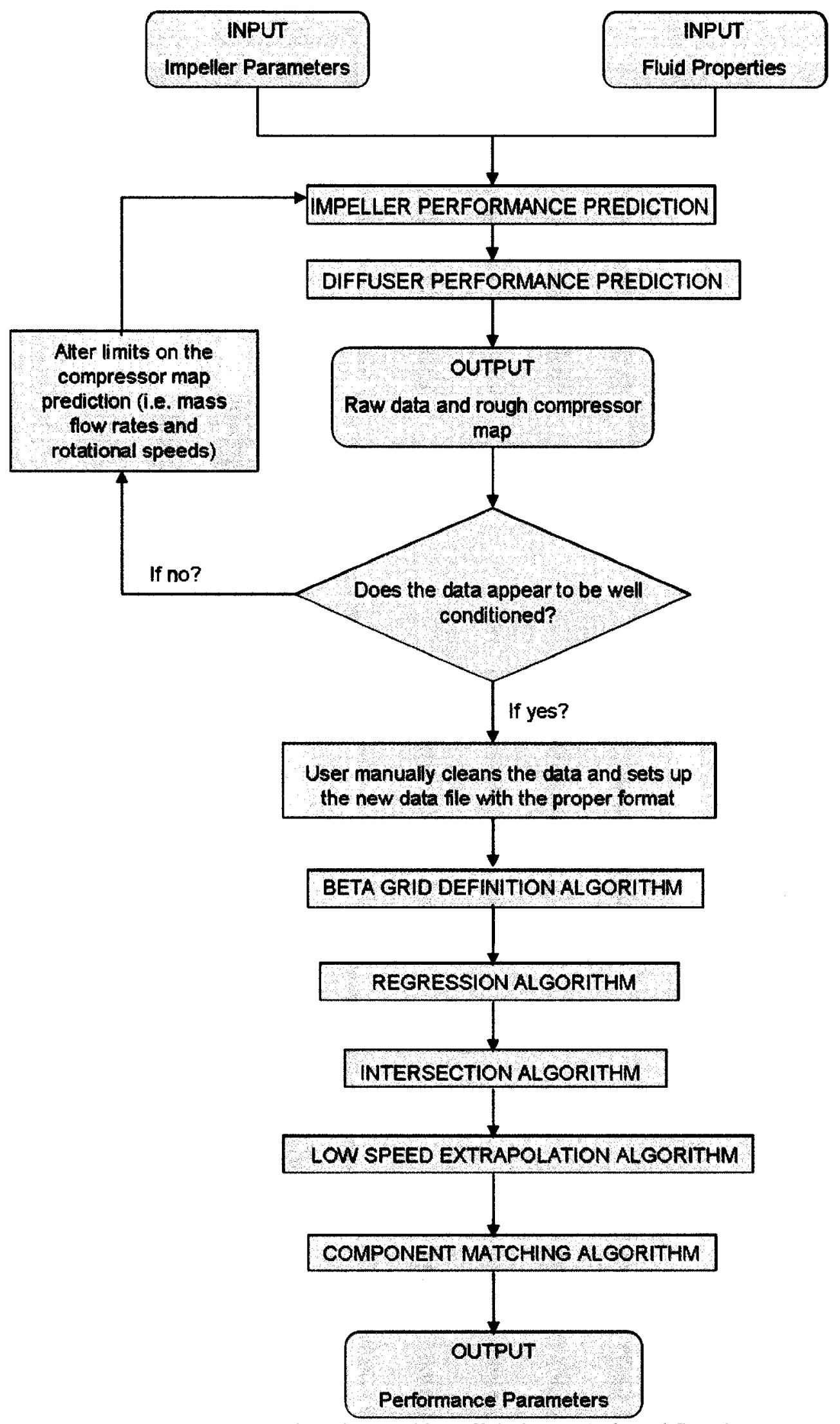

Figure 3.1 Compressor synthesis and gas turbine off-design analysis tool flowchart. 
Initially, the program determines the values for several empirical coefficients that vary with flow condition and impeller size which are used in the model. The values of these coefficients and the criteria for their selection are discussed explicitly in section 3.1.2. These are selected by determining those values of empirical coefficients which generate a pressure ratio which corresponds as closely as possible to the design pressure ratio at the design rotational speed. The user is also able to circumvent this aspect of the model, and directly supply the values of empirical coefficients, since this is based highly on user experience. These coefficients are then used in all subsequently calculations. The program then asks the user for the range of rotational speeds to calculate, as well as the step size for rotational speed and the range of mass flow rates to calculate with the step size for mass flow rate. The program then undergoes the calculations outlined in the impeller model, determining the inlet conditions, the jet conditions, the wake conditions, the mixed-out state conditions as well as the diffuser outlet conditions (if applicable).

The program then requires manual user input to continue with the off-design analysis. As will be outlined below, the impeller model solutions tend to diverge at low rotational speeds and low mass flow rates. Therefore, the user is required to determine what data is good and is useful to keep, and which pieces of data can be discarded of all the data points calculated by the off-design model. This part of the model is dependant upon designer experience and is, at present, a drawback of the two-zone modeling process. Figure 3.2 gives an indication of what can be termed good and bad data. 


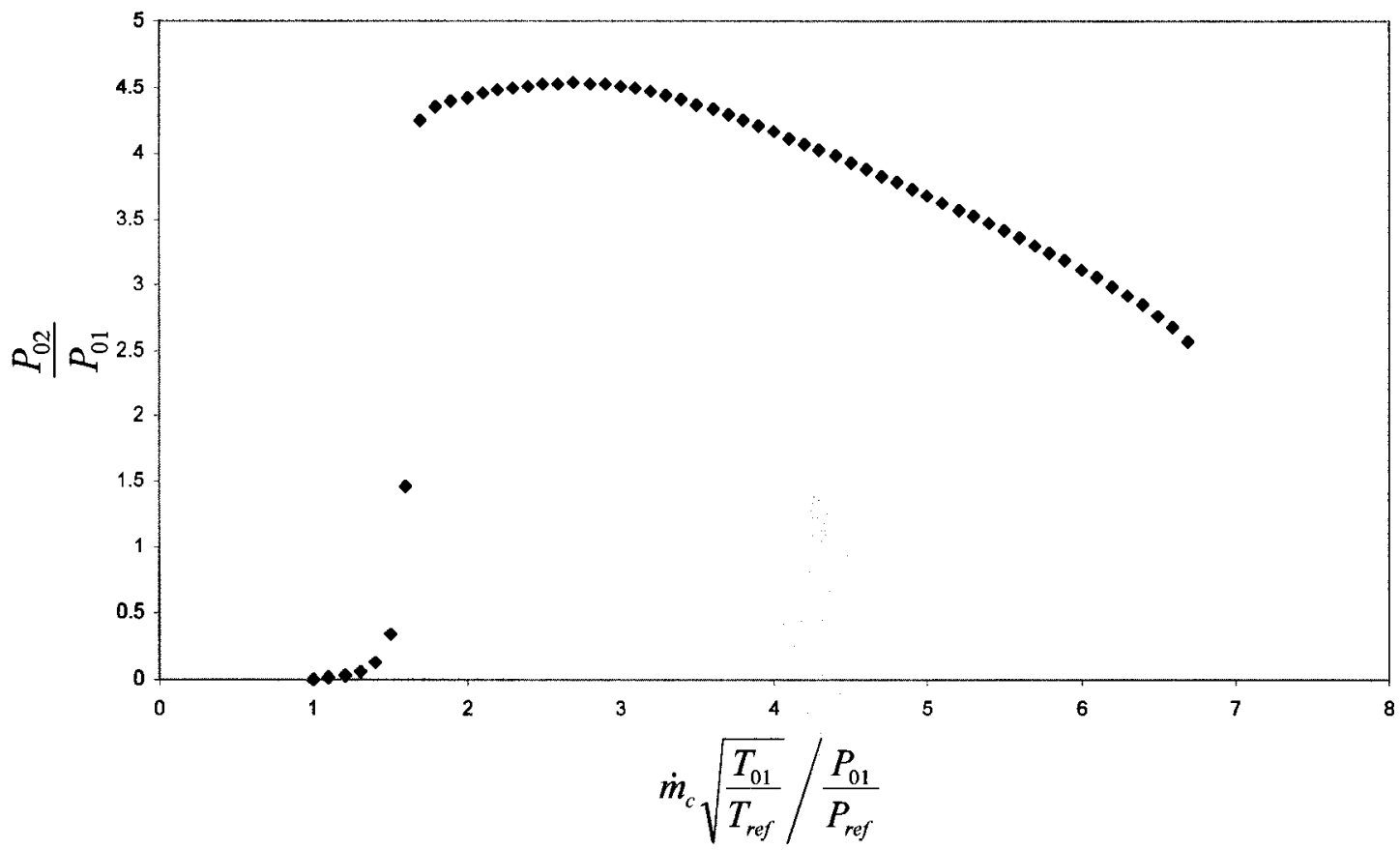

Figure 3.2 Sample data generated by the compressor off design performance model.

Determining what data is acceptable to keep and which pieces of data can be discarded requires the user to have a basic understanding of what a compressor characteristics should look like. Figure 3.2 shows the design speed characteristic for the sample impeller described by McKain and Holbrook (1997) which is outlined further in section 4.1. Data is generated by the compressor performance prediction algorithm between corrected mass flows of $1.0 \mathrm{~kg} / \mathrm{s}$ and $6.7 \mathrm{~kg} / \mathrm{s}$. While most of the data can be retained, the data generated below a corrected mass flow rate of $1.7 \mathrm{~kg} / \mathrm{s}$ clearly does not follow the general trend of the characteristic and, as such, can be discarded once all compressor data has been generated. This is indicative of what can be expected for bad data; the user should feel comfortable discarding outlier data, that is, data which does not follow the general characteristics. 
The data is then processed into a new text file (with a format outlined below) for use in subsequent algorithms of the model. From this point, no user intervention is required.

Data is then fed into the beta grid formation algorithm which processes the data and generates a beta grid which encapsulates all the data in the map. A beta grid is a series of auxiliary coordinates which are overlaid on the compressor map to provide unique intersections with the lines of constant speed. These auxiliary coordinates, which are used often in map scaling techniques, will be applied to the low speed extrapolation algorithm which will be outlined in section 3.3. Figure 3.3 shows a coarse beta grid for illustration purposes. Red lines correspond to the beta lines, where the blue curves correspond to the actual map characteristics. Ten beta lines are included in this map, which should be noted as a very coarse grid. It is recommended that between one hundred and five hundred beta lines are used in conjunction with the low speed extrapolation technique. The reader is referred to section 3.4 for further discussion regarding beta lines. 


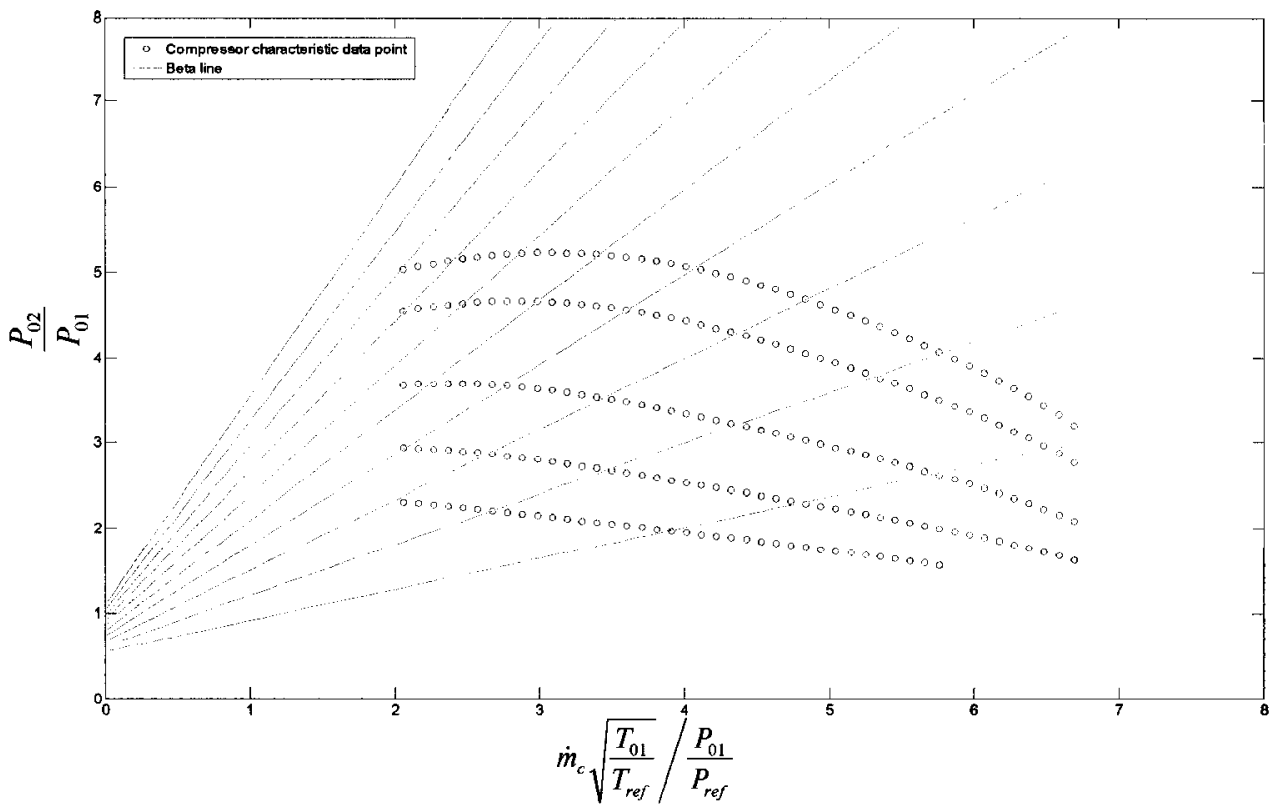

Figure 3.3 Sample beta grid overlaid on a compressor map.

From this point, a regression is performed on the data to generate curves corresponding to the compressor characteristics. Once the intersection of the beta lines and the compressor characteristics has been performed, a low speed extrapolation is performed to determine the sub-idle region based upon user inputs. Finally, regressions are performed on the extrapolated data and these regressions are combined with those performed on impeller generated curves to provide a complete compressor map.

A number of assumptions are included in this model, some of which are discussed explicitly in the following sections. Air was assumed to behave as a perfect gas with a constant specific heat ratio. Furthermore, it was assumed that flow enters the centrifugal compressor in an axial direction without circumferential or radial variations. Also it was assumed that the difference between ambient conditions and the inlet stagnation pressure 
and temperature was negligible since no information regarding the engine inlet design was available.

\subsection{Impeller Performance Prediction}

The model which is used to determine the impeller performance has been adapted from the papers published by Oh et al. (2002) regarding a new version of the two-zone model and Japikse (1985) outlining a more traditional two-zone modelling approach. Used in conjunction with newer loss correlations to account for recirculation and disk friction loss, the model described by Oh et al. (2002) demonstrated a high degree of accuracy for a wide range of pressure ratios in a relatively simple algorithm. As mentioned in previous sections, the main concept of the model is to split the impeller flow into two separate distinct zones: an isentropic jet flow which is able to deviate from the impeller wall (thus accounting for slip in the impeller) and a non-isentropic wake flow which follows the wall perfectly; this zone accounts for the internal losses in the system. An example of the two-zone model can be seen in Figure 2.8. Three main assumptions are required when implementing such a model: 1) Impeller tip static pressure for the jet and wake flows are identical, this is known as the unloaded tip condition and is required for closure of the equation system, 2) no mixing of the jet and wake flow in the impeller channel and 3) the wake zone follows the blade exactly. Wake mass flow fraction is assumed constant, but can be altered by the user, allowing for the investigation of the effect of variable wake mass flow on impeller performance. 


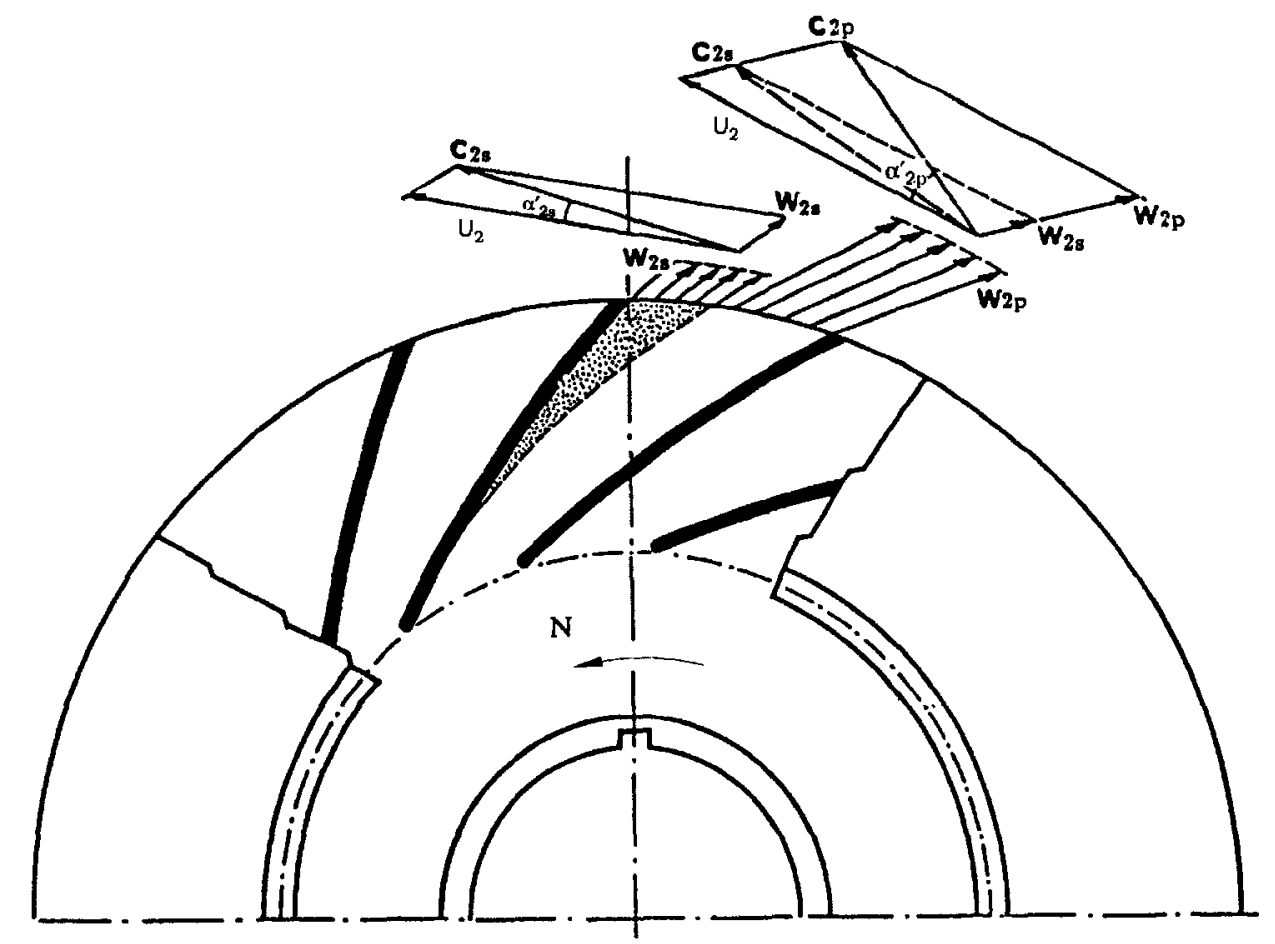

Figure 3.4 Schematic of the two-zone model impeller exit flow.

Required parameters for the model include:

- Inlet Stagnation Pressure $\left(\mathrm{P}_{01}\right)$

- Inlet Stagnation Temperature $\left(\mathrm{T}_{01}\right)$

- Design mass flow rate $(\dot{m})$

- Design speed $\left(\mathrm{N}_{\text {des }}\right)$

- Inducer hub radius $\left(\mathbf{r}_{1 h}\right)$

- Inducer tip radius $\left(\mathrm{r}_{1 \mathrm{t}}\right)$

- Outlet radius $\left(\mathrm{r}_{2}\right)$

- Blade height at inlet $\left(b_{1}\right)$

- Blade height at outlet $\left(b_{2}\right)$ 
- Backsweep angle $\left(\beta_{2 b l a d e}\right)$

- Number of blades (Z)

- Blade thickness at outlet $\left(\mathrm{t}_{2 \mathrm{~b}}\right)$

- Inlet hub blade angle $\left(\beta_{1 \text { blade }_{h}}\right)$

- Inlet tip blade angle $\left(\beta_{\text {lblade }}\right)$

- Design pressure ratio $\left(\mathrm{PR}_{\text {des }}\right)$

For an example of the format of the input file, the user is referred to Appendix B. A flowchart describing the overall impeller model algorithm is shown in Figure 3.5. 


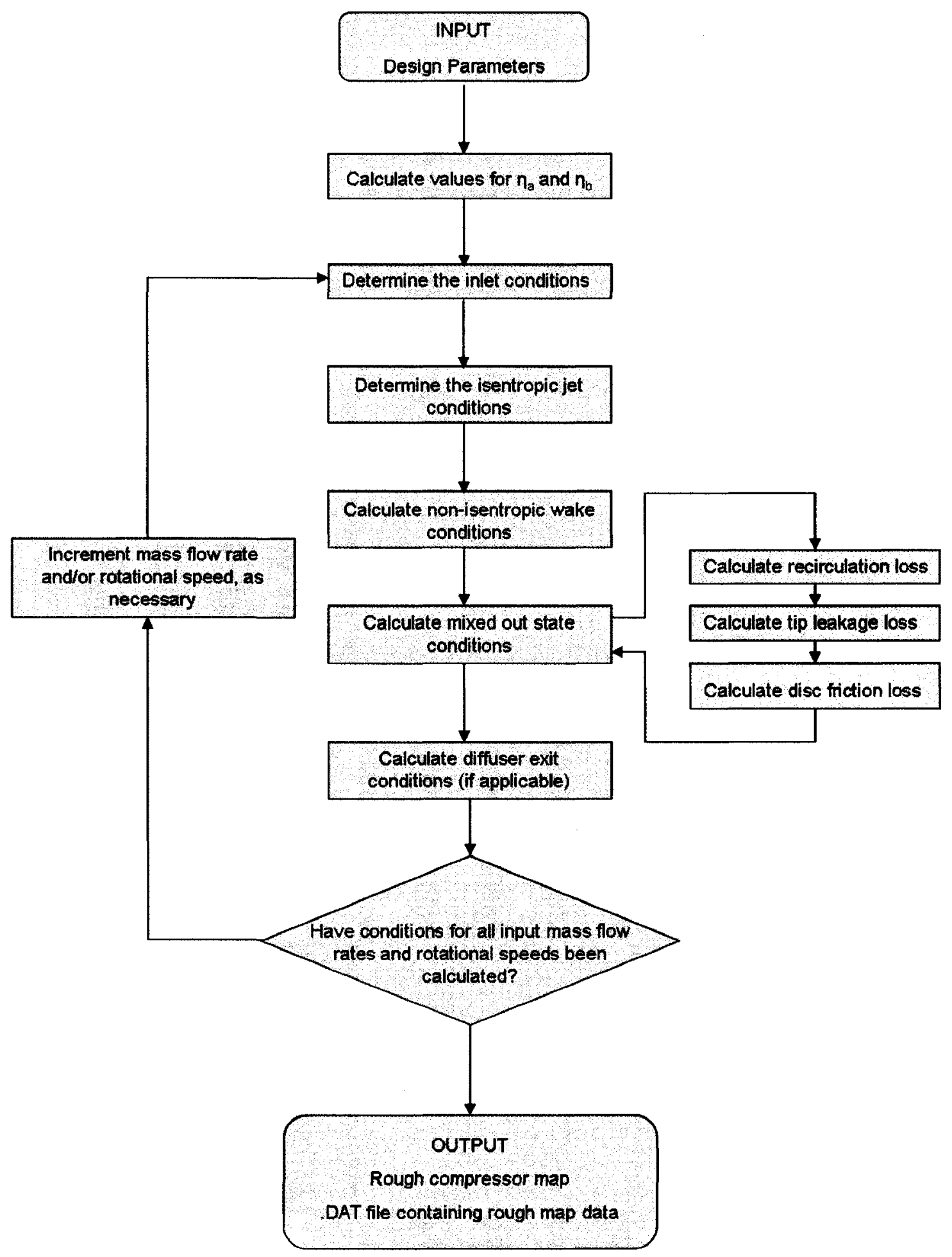

Figure 3.5 Impeller model flowchart. 


\subsubsection{Inlet Zone Calculations}

To accommodate the two-zone model, the inlet conditions must be determined at the impeller inlet tip. Once the inlet blade tip velocity has been determined inlet calculation continue by estimating the inlet static density. This requires an iterative solution since the inlet density is not known. The following set of equations is used to determine the inlet conditions:

$$
\begin{aligned}
& \omega=\frac{2 \pi N}{60} \\
& \rho_{01}=\frac{P_{01}}{R T_{01}} \\
& A_{1}=\pi\left(r_{1 t}^{2}-r_{1 h}^{2}\right) \\
& U_{1 t}=\omega r_{1 t} \\
& C_{x 1}=\frac{\dot{m}}{\rho_{1} A_{1}} \\
& T_{1}=T_{01}-\frac{C_{x 1}^{2}}{2 C_{p}} \\
& \rho_{1}=\frac{\left(1+\frac{\gamma-1}{2} M_{1}^{2}\right)^{\frac{1}{\gamma-1}}}{\left(\frac{T_{01}}{T_{1}}-1\right)} \\
& M_{1}=\sqrt{2-1}
\end{aligned}
$$


where:

$\omega$ is the impeller angular velocity in radians per second

$N$ is the rotational speed in RPM

$\rho$ denotes air density

$A$ denotes the flow area

$C_{x 1}$ denotes inlet absolute axial velocity

$T$ corresponds to temperature

$M_{l}$ corresponds to inlet Mach number

Equations 3.5 through 3.8 are iterated upon until convergence, thereby determining the inlet conditions. From this point, the remainder of the required inlet conditions is determined as follows:

$$
\begin{aligned}
& \beta_{1}=\tan ^{-1}\left(\frac{U_{1 t}}{C_{x 1}}\right) \\
& W_{1 t}=\sqrt{C_{x 1}^{2}+U_{1 t}^{2}} \\
& P_{1}=\frac{P_{01}}{\left[1+\frac{\gamma-1}{2} M_{1}^{2}\right]^{\frac{\gamma}{\gamma-1}}} \\
& M_{1 \text { rel }}=\frac{W_{1 t}}{\sqrt{\gamma R T_{1}}} \\
& P_{01 r e l}=P_{1}\left[1+\frac{\gamma-1}{2} M_{1 \text { rel }}^{2}\right]^{\frac{\gamma}{\gamma-1}}
\end{aligned}
$$


where:

$\beta_{1}$ denotes the inlet relative flow angle

$W_{1 t}$ denotes the inlet tip relative velocity

$P$ denotes pressure

rel subscript denotes a relative flow condition

With the above quantities determined, all conditions are known to determine the conditions in the isentropic jet.

\subsubsection{Diffusion Ratio Estimation}

Predicting the diffusion characteristics accurately is a small, but important aspect of a two-zone model. As mentioned, the diffusion ratio is the parameter which links the inlet flow conditions to the outlet jet flow:

$$
D R=\frac{W_{1 t}}{W_{2 j}}
$$

where:

$W_{2 j}$ denotes the outlet jet relative velocity

Therefore, with proper estimation of the diffusion ratio, it is possible to compute the outlet jet relative velocity, as required for the jet zone calculation procedure. This model utilizes the two-element in series (TEIS) model developed by Japikse (1996). 


$$
D R^{2}=\frac{1}{\left(1-\eta_{a} C_{p a, i}\right)\left(1-\eta_{b} C_{p b, i}\right)}
$$

where,

$$
\begin{aligned}
& C_{p a, i}=1-\left(\frac{\cos \beta_{1}}{\cos \beta_{1 b l}}\right)^{2} \\
& C_{p b, i}=1-\left(\frac{A_{t h}}{A_{e}}\right)^{2} \\
& A_{t h} \approx \frac{A_{i n}}{Z} \sin \left(90^{\circ}-\beta_{1 b}\right)
\end{aligned}
$$

where:

$\eta_{a}$ denotes the impeller inlet effectiveness

$\eta_{b}$ denotes the impeller passage effectiveness

$C_{p a, i}$ denotes the ideal pressure recovery coefficient of the inlet

$C_{p b, i}$ denotes the ideal pressure recovery coefficient of the impeller passage

$A_{t h}$ denotes the passage throat area

$A_{\text {in }}$ denotes passage inlet area

$A_{e}$ denotes impeller passage exit area

$b l$ subscript denotes a blade condition 
The ideal pressure recovery coefficients can be calculated directly from the known impeller geometry and inlet flow conditions. The ideal pressure recovery coefficient of the passage is constant over the whole range of flow conditions. However, the ideal pressure recovery coefficient of the inlet varies with the inlet flow angle. Empirical coefficients $\eta_{a}$ and $\eta_{b}$ are effectiveness parameters of the inlet and the impeller passage and remain constant over the range of flow conditions. Japikse (1996) provides estimations of the ranges of acceptable values for $\eta_{a}$ and $\eta_{b}$, however it should be noted that those mentioned are merely guidelines. Japikse (1996) also provides a reasonable range of expected values for wake mass flow fraction as a part of the TEIS model. Table 3.1, adapted from Japikse (1996) gives the overall guidelines on the selection of the values for the empirical coefficients $\eta_{a}$ and $\eta_{b}$ as well as rough estimate for the wake mass flow fraction, $\chi$.

Table 3.1 Empirical coefficients for the calculation of diffusion ratio in the TEIS model.

\begin{tabular}{|l|c|c|c|}
\hline \multicolumn{1}{|c|}{ Case } & $\eta_{a}$ & $\eta_{b}$ & $\chi$ \\
\hline $\begin{array}{l}\text { Large, well designed rotors } \\
\left(10 \text { " to } 12 \text { " } \mathrm{D}_{2}\right)\end{array}$ & 0.9 to 1.1 & 0.4 to 0.6 & 0.1 to 0.2 \\
\hline $\begin{array}{l}\text { Medium size, well designed } \\
\left.\text { (4" to } 10 " \mathrm{D}_{2}\right)\end{array}$ & 0.8 to 0.9 & 0.3 to 0.5 & 0.15 to 0.25 \\
\hline $\begin{array}{l}\text { Medium size, ordinary design } \\
\left(4 " \text { to } 10 " \mathrm{D}_{2}\right)\end{array}$ & 0.6 to 0.8 & 0.0 to 0.4 & 0.20 to 0.30 \\
\hline $\begin{array}{l}\text { Small or poorly designed } \\
\left(<4 \text { " } \mathrm{D}_{2}\right)\end{array}$ & 0.4 to 0.6 & -0.3 to +0.3 & 0.25 to 0.35 \\
\hline
\end{tabular}

The problem at this point was the determination of which values to use within the guidelines. The problem here lies in the determination of the empirical coefficients within 
the TEIS model. A preliminary two-zone model calculation is therefore performed to determine which values of $\eta_{a}$ and $\eta_{b}$ match the design pressure ratio at the design speed and mass flow rate. Once these values are determined, they are subsequently used in all two-zone model calculations. With the diffusion ratio determined, the impeller exit relative jet velocity can be determined for any mass flow rate by Equation 3.14.

\subsubsection{Jet Zone Model}

With the inlet conditions determined, all the required quantities are passed into a module to calculate the conditions at the exit of the impeller, specifically within the isentropic jet. This analysis simplifies the process proposed by Oh et al. (2002) by leaving the wake mass flow fraction, $\chi$, constant over the operating conditions. While experiment indicates that the constancy of $\chi$ is not entirely true, it introduces both complexity and instability to the model and furthermore, does not seem to influence the accuracy of the results to a large degree. Starting with an assumed value for the wake mass flow fraction, $\chi$, the following jet zone quantities are determined:

$$
\begin{aligned}
& A_{i n}=\frac{2 \pi\left(r_{1 t}-r_{1 h}\right) b_{1}}{Z} \\
& A_{t h}=A_{i n} \sin \left(90^{\circ}-\beta_{1 b l}\right) \\
& A_{e}=\frac{2 \pi r_{2} b_{2}}{Z} \\
& h_{01}=\frac{\gamma R}{\gamma-1} T_{01}
\end{aligned}
$$




$$
\begin{aligned}
& h_{2 j}=h_{01}-\frac{W_{2 j}^{2}}{2}+\frac{U_{2}^{2}}{2} \\
& T_{2 j}=\frac{\gamma-1}{\gamma R} h_{2 j} \\
& P_{2 j}=P_{1}\left(\frac{T_{2 j}}{T_{1}}\right)^{\frac{\gamma}{\gamma-1}} \\
& \rho_{2 j}=\frac{P_{2 j}}{R T_{2 j}} \\
& C_{2 j}=\sqrt{C_{m 2 j}^{2}+C_{\theta 2 j}^{2}}=U_{2}-W_{2 j} \sin \beta_{2 j} \\
& f_{j j}=5.73 \times 10^{-6}\left(90^{\circ}-\beta_{2 b}\right)^{\frac{5}{2}} \\
& A_{g e o}=2 \pi r_{2} b_{2}-\frac{Z b_{2} t_{2 b l}}{\cos \left(\beta_{2 b l}\right)} \\
& A_{2 j}=1-f_{j} \frac{\beta_{2 j}}{r_{2} b_{2} Z} \\
& A_{g e o}(1-\varepsilon) \\
& A_{2 j}
\end{aligned}
$$




$$
\begin{aligned}
& \alpha_{2 j}=\tan ^{-1}\left(\frac{C_{\theta 2 j}}{C_{m 2 j}}\right) \\
& T_{02 j}=T_{2 j}+\frac{\gamma-1}{2 \gamma R} C_{2 j}^{2} \\
& P_{02 j}=P_{2 j}\left(\frac{T_{02 j}}{T_{2 j}}\right)^{\frac{\gamma}{\gamma-1}}
\end{aligned}
$$

where:

$b_{1}$ denotes the inlet blade height

$\beta_{1 b l}$ denotes inlet blade angle

$\gamma$ denotes specific heat ratio

$U_{2}$ denotes outlet tip speed

$h_{2 j}$ denotes the static enthalpy of the jet

$f_{j}$ is an empirical coefficient described by Oh et al. (2002) for determining the effect of backsweep on the slip factor $\mu_{j}$ denotes slip $\varepsilon$ denotes the wake area fraction $A_{\text {geo }}$ denotes impeller geometric area $Z$ denotes the number of blades $C_{m 2 j}$ denotes absolute meridional jet velocity $C_{\theta 2 j}$ denotes absolute tangential jet velocity $\alpha_{2 j}$ denotes jet outlet absolute flow angle 
Equations 3.19 through 3.37 allow for the determination of the necessary quantities required for the solution of the jet. Furthermore, Equations 3.28 and 3.27 are empirical relationships developed by Whitfield (1974) and Oh et al. (2002), respectively. Whitfield concluded that the slip factor for determining impeller outlet conditions varies with the degree of backsweep in the impeller; a modified form of the slip factor is shown in Equation 3.28, solving for the outlet relative flow angle of the isentropic jet.

From this point, any necessary quantities can be passed to the wake calculation model where the two-zone model calculations continue.

\subsubsection{Wake Zone Model}

The wake zone is the second area of the two-zone model which is required for the mixed-out state calculations. The analysis is mainly based upon the wake zone equations proposed by Japikse (1985). Three assumptions are required for this analysis: 1) that the wake flow follows the blade exactly, 2) that front cover friction is negligible and 3) that the exit static pressure of the wake equals the exit static pressure of the jet. While it is not precisely true that the front cover friction is negligible, an estimation of this value requires quantities to be determined at the mixed-out state, a state which is not known at this point in the calculation procedure. While an iterative loop could be added between the wake zone and the mixed-out state to estimate this front cover friction, it counters the simplicity of the model. Furthermore, it does not seem to be required to produce reasonable results as indicated by the results shown in sections 4.2 through 4.5 . 
To continue with the wake zone model, the following quantities are required to be passed from the jet zone: jet exit static pressure $\left(P_{2 j}\right)$, outlet impeller speed $\left(U_{2}\right)$, inlet impeller speed $\left(U_{l t}\right)$, impeller exit area excluding blade blockage $\left(A_{\text {geo }}\right)$, jet exit area $\left(A_{2 j}\right)$, jet exit static temperature $\left(T_{2 j}\right)$, inlet relative stagnation temperature $\left(T_{01 r e l}\right)$, the mass flow rate for the given calculation $(\dot{m})$, wake mass flow fraction $(\chi)$, blade exit angle $\left(\beta_{2 b l}\right)$, jet exit tangential absolute velocity $\left(C_{\theta 2 j}\right)$, inlet and exit impeller geometry, jet exit meridional absolute velocity $\left(C_{m 2 j}\right)$, jet exit relative flow angle $\left(\beta_{2}\right)$, number of impeller blades $(Z)$ and inlet axial absolute velocity $\left(C_{x l}\right)$. The calculations begin with one of the assumptions of the model; the exit static pressures of the jet and wake zones are identical. This is required to close the system of equations. The following describes the order that the calculations should be performed to achieve convergence:

$$
\begin{aligned}
& h_{02 w}=h_{01}+\frac{\dot{W}_{f c}}{\dot{m}}=h_{01} \\
& P_{2 j}=P_{2 w} \quad \text { (unloaded tip condition) } \\
& T_{2 w_{\text {guess }}}=T_{2 j} \\
& \rho_{2 w}=\frac{P_{2 w}}{R T_{2 w_{\text {guess }}}} \\
& C_{m 2 w}=\frac{\frac{\dot{m}}{A_{g e o}}-\rho_{2 j} C_{m 2 j}(1-\varepsilon)}{\rho_{2 w} \varepsilon}
\end{aligned}
$$




$$
\begin{aligned}
& W_{2 w}=\frac{C_{m 2 w}}{\cos \beta_{2 b}} \\
& h_{2 w}=h_{02 w}-\frac{W_{2 w}^{2}}{2}+\frac{U_{2}^{2}}{2} \\
& T_{2 w}=\frac{\gamma-1}{\gamma R} h_{2 w}
\end{aligned}
$$

where:

$h_{02 w}$ denotes the total enthalpy of the wake

$\dot{W}_{f c}$ denotes front cover friction

$P_{2 w}$ denotes the static pressure of the wake

$C_{m 2 w}$ denotes the meridional component of absolute velocity of the wake

$W_{2 w}$ denotes the exit wake relative velocity

$h_{2 w}$ denotes the static enthalpy of the wake

$T_{2 w}$ denotes the static temperature of the wake

Equations 3.41 through 3.45 are iterative and the calculation continues until the value for exit wake static temperature converges within a prescribed tolerance. Once the iteration loop converges, the wake exit velocity triangle can be established.

$$
\begin{aligned}
& C_{\theta 2 w}=U_{2}-C_{m 2 w} \tan \beta_{2 b l} \\
& C_{2 w}=\sqrt{C_{\theta 2 w}^{2}+C_{m 2 w}^{2}}
\end{aligned}
$$




$$
\begin{aligned}
& T_{02 w}=T_{2 w}+\frac{\gamma-1}{2 \gamma R} C_{2 w}^{2} \\
& P_{02 w}=P_{2 w}\left(\frac{T_{02 w}}{T_{2 w}}\right)^{\frac{\gamma}{\gamma-1}}
\end{aligned}
$$

where:

$$
\begin{aligned}
& C_{\theta 2 w} \text { denotes the wake exit absolute tangential velocity } \\
& C_{2 w} \text { denotes the wake exit absolute velocity } \\
& T_{02 w} \text { denotes the wake exit total temperature } \\
& P_{02 w} \text { denotes the wake exit total pressure }
\end{aligned}
$$

Again, any necessary quantities can be passed to the next module. Once all wake zone quantities have been determined, they can be combined with the jet zone quantities to determine the mixed-out state.

\subsubsection{Mixed-Out State Model}

The final step in the impeller model involved determining the mixed-out state from the jet and wake zone data. It is assumed that mixing occurs very rapidly after the exit of the impeller, before the flow can enter into the vaneless diffuser. The following set of equations describes a method for determining the effective thermodynamic state at the exit of the impeller; many quantities are determined on a mass-averaged basis of the jet and wake zone. External loss correlations are supplied to determine the losses not 
inherently accounted for the two-zone model, namely the disk friction loss, the recirculation loss and the tip leakage loss.

To complete the mixed-out state analysis, the following quantities need to be supplied: wake mass flow fraction $(\chi)$, impeller geometry, absolute tangential jet velocity $\left(C_{\theta 2 j}\right)$, absolute tangential wake velocity $\left(C_{\theta 2 w}\right)$, absolute meridional jet velocity $\left(C_{m 2 j}\right)$, absolute meridional wake velocity $\left(C_{m 2 w}\right)$, relative inlet tip velocity $\left(W_{1 t}\right)$, relative exit jet velocity $\left(W_{2 j}\right)$, relative exit wake velocity $\left(W_{2 w}\right)$, impeller tip velocity $\left(U_{2}\right)$, inlet axial velocity $\left(C_{x l}\right)$, jet static pressure $\left(P_{2_{j}}\right)$, jet stagnation temperature $\left(T_{2 j}\right)$, wake stagnation temperature $\left(T_{2 w}\right)$, inlet density $\left(\rho_{1}\right)$ and mass flow rate $(\dot{m})$. The analysis proceeds in the following fashion:

$$
\begin{aligned}
& C_{\theta 2 \text { mix }}=(1-\chi) C_{\theta 2 j}+\chi C_{\theta 2 w} \\
& T_{02 \text { mix }}=(1-\chi) T_{02 j}+\chi T_{02 w} \\
& C_{\text {m2mix }}=\frac{b-\sqrt{b^{2}-4 a c}}{2 a}
\end{aligned}
$$

where:

$$
\begin{gathered}
a=\frac{\dot{m}}{\pi D_{2} b_{2}} \frac{\gamma+1}{2 \gamma} \\
b=\frac{P_{2 j} A_{g e o}}{\pi D_{2} b_{2}}+\frac{\dot{m}}{\pi D_{2} b_{2}}\left[(1-\chi) C_{m 2 j}+\chi C_{m 2 w}\right] \\
c=\frac{\dot{m}}{\pi D_{2} b_{2}}\left(T_{02 m i x}-\frac{\gamma-1}{2 \gamma} C_{\theta 2 m i x}^{2}\right)
\end{gathered}
$$




$$
\begin{gathered}
C_{2 m i x}=\sqrt{C_{r 2 m i x}^{2}+C_{\theta 2 m i x}^{2}} \\
T_{2 m i x}=T_{02 m i x}-\frac{\gamma-1}{2 \gamma R} C_{2 m i x}^{2} \\
P_{2 m i x}=\frac{P_{2 j} A_{g e o}}{\pi D_{2} b_{2}}+\frac{\dot{m}}{\pi D_{2} b_{2}}\left[(1-\chi) C_{m 2 j}+\chi C_{m 2 w}-C_{m 2 m i x}\right] \\
\rho_{2 m i x}=\frac{P_{2 m i x}}{R T_{2 m i x}} \\
\alpha_{2}=\cos ^{-1}\left(\frac{C_{m 2 m i x}}{C_{2 m i x}}\right) \\
T_{02 m i x}=(1-\chi) T_{02 j}+\chi T_{02 w}+\frac{\gamma-1}{\gamma R \dot{m}}\left(\dot{W}_{d f}+\dot{W}_{r c}+\dot{W}_{t l}\right)
\end{gathered}
$$

where the power terms represent the external losses which will be discussed in section 3.1.6. The nomenclature for Equations 3.50 through 3.61 are as follows:

$C_{\theta 2 m i x}$ denotes mixed-out state tangential velocity

$T_{02 m i x}$ denotes mixed-out state total temperature

$C_{m 2 m i x}$ denotes mixed-out state meridional velocity

$D_{2}$ denotes impeller outlet diameter

$b_{2}$ denotes impeller outlet blade height

$C_{2 m i x}$ denotes outlet absolute velocity

$T_{2 m i x}$ denotes mixed-out state static temperature

$\rho_{2 m i x}$ denotes the mixed-out state static density

$\alpha_{2}$ denotes the outlet absolute flow angle 
$\dot{W}_{d f}$ denotes the extra power input due to disc friction

$\dot{W}_{r c}$ denotes the extra power input due to recirculation losses

$\dot{W}_{t l}$ denotes the extra power input due to tip leakage losses

Equations 3.52 through 3.61 are iterated upon until the mixed-out stagnation temperature converges within a prescribed tolerance. Once this value has been established, mixed-out conditions for pressure, isentropic stagnation temperature and finally impeller efficiency can be calculated.

$$
\begin{aligned}
& W_{2 m i x}=(1-\chi) W_{2 j}+\chi W_{2 w} \\
& \beta_{2 m i x}=(1-\chi) \beta_{2 j}+\chi \beta_{2 w} \\
& P_{02 m i x}=P_{2 m i x}\left(\frac{T_{02 m i x}}{T_{2 m i x}}\right)^{\frac{\gamma-1}{\gamma}} \\
& T_{02 s}=T_{01}\left(\frac{P_{02 m i x}}{P_{01}}\right)^{\frac{\gamma-1}{\gamma}} \\
& \eta=\frac{T_{02 s}-T_{01}}{T_{02 m i x}-T_{01}}
\end{aligned}
$$

where:

$W_{2 m i x}$ denotes the mixed-out state relative velocity

$\beta_{2 m i x}$ denotes the mixed-out state relative flow angle

$P_{02 m i x}$ denotes the mixed-out state total pressure

$T_{02 s}$ denotes isentropic total pressure of the impeller exit 
$\eta$ denotes compressor isentropic efficiency

Calculations begin with the inlet algorithm; necessary quantities are passed to the jet zone algorithm followed by the wake zone algorithm. Finally, all necessary quantities are passed to the mixed-out state algorithm where the final outlet conditions are calculated. This process then repeats for each combination of impeller speed and mass flow rate desired to complete the impeller map.

It is recommended that the overall method be placed into a computer code where the design point data is supplied to the program by means of a text file. The user can then select the range of rotational speeds and the range of mass flow rates desired, as well as a step size between adjacent calculation points. In this fashion, the rough impeller map can be established within seconds. For completeness, Appendix A contains a listing of the source code developed by the author for such purposes.

\subsubsection{Loss Models}

The two-zone model consists most fundamentally of an isentropic jet in the centre of a loss containing wake. Although the model inherently accounts for an entropy increase (internal losses) in the wake, it fails to account for the external loss mechanisms associated with operation. The three external losses which need to be accounted for are: 1) recirculation loss, 2) disc friction loss and 3) tip leakage loss. 


\subsubsection{Recirculation Loss}

Recirculation loss is the reduction in efficiency due to high pressure flow returning to the inlet through the clearance gap between the impeller and the casing. This flow occurs due to the high pressure flow at the impeller exit and the low pressure flow at the impeller inlet. Since flow is returning to the inlet, some of the air moving through the centrifugal impeller is not translated to useful pressure rise, leading to a reduction in efficiency.

According to Oh et al. (1997) recirculation loss dominates the external losses at all flow rates and speed, thus it is important to obtain the most accurate estimation possible. Extra work input is required to overcome internal and external losses, especially at low mass flow rates (Aungier, 2000). It is believed that this input is associated with the flow of the working fluid through the clearance gap to the inlet. As such, not all of the fluid flow moving through the impeller goes towards increasing the pressure ratio resulting in a decrease in isentropic efficiency.

Oh et al. (1997) proposed a model to improve the accuracy of recirculation loss calculations and subsequently demonstrated its applicability over other recirculation models. Many parameters are required for input, however no extra inputs are required other than those calculated by, or supplied to, the two-zone impeller model. The selected algorithm requires: exit absolute flow angle $\left(\alpha_{2}\right)$, outlet impeller speed $\left(U_{2}\right)$, inlet relative tip velocity $\left(W_{l t}\right)$, outlet relative jet velocity $\left(W_{2 j}\right)$, outlet relative wake velocity 
$\left(W_{2 w}\right)$, outlet radius $\left(r_{2}\right)$, inlet tip radius $\left(r_{I t}\right)$, wake mass flow fraction $(\chi)$, outlet wake relative flow angle $\left(\beta_{2 w}\right)$, outlet jet relative flow angle $\left(\beta_{2 j}\right)$, number of impeller blades $(Z)$, outlet mixed state relative velocity $\left(W_{2 m i x}\right)$ and outlet mixed state relative flow angle $\left(\beta_{2 m i x}\right)$. The algorithm is as follows:

$$
\begin{aligned}
& \Delta h_{\text {euler }}=U_{2}\left[U_{2}-\left(W_{2 m i x} \sin \beta_{2 m i x}\right)\right] \\
& D_{f}=1-\frac{W_{2}}{W_{1 t}}+\frac{0.75 \Delta h_{\text {euler }} / U_{2}^{2}}{\frac{W_{2}}{W_{1 t}}\left[\left(\frac{Z}{\pi}\right)\left(1-\frac{r_{1 t}}{r_{2}}\right)+2 \frac{r_{1 t}}{r_{2}}\right]} \\
& \Delta h_{r c}=8 \times 10^{-5} \sinh \left(3.5 \alpha_{2}^{3}\right) D_{f}^{2} U_{2}^{2} \\
& \dot{W}_{r c}=\dot{m} \Delta h_{r c}
\end{aligned}
$$

where:

- $\Delta h_{\text {euler }}$ denotes the Euler enthalpy rise which corresponds to the ideal enthalpy rise within the impeller

- $D_{f}$ denotes a modified diffusion factor

- $\Delta h_{r c}$ denotes the enthalpy rise due to recirculation

The value for recirculation power is then passed to Equation 3.61 in the mixed-out state analysis. 
A second external loss mechanism occurs through disk friction and windage. Both Oh et al. (1997) and Aungier (2000) suggest that the best available model for disk friction is that proposed by Daily and Nece (1960). Inputs to the model are: inlet and outlet static density $\left(\rho_{1}, \rho_{2}\right)$, outlet radius $\left(r_{2}\right)$, outlet impeller speed $\left(U_{2}\right)$, mass flow rate $(\dot{m})$ and outlet static temperature $\left(T_{2}\right)$. The calculation is as follows:

$$
\begin{aligned}
& \bar{\rho}=\frac{\rho_{1}+\rho_{2}}{2} \\
& \mu_{2}=1.71 \times 10^{-5}\left[\left(\frac{T_{2}}{273}\right)^{1.5}\left(\frac{273+110.4}{T_{2}+110.4}\right)\right] \\
& \operatorname{Re}_{d f}=\frac{U_{2} r_{2} \rho_{2}}{\mu_{2}} \\
& f_{d f}= \begin{cases}\frac{2.67}{\operatorname{Re}_{d f}^{\frac{1}{2}}} & \operatorname{Re}_{d f}<3 \times 10^{5} \\
\frac{0.0622}{\operatorname{Re}_{d f}^{0.2}} & \operatorname{Re}_{d f} \geq 3 \times 10^{5}\end{cases}
\end{aligned}
$$

$$
\begin{aligned}
& \Delta h_{d f}=f_{d f} \frac{\bar{\rho} r_{2}^{2} U_{2}^{3}}{4 \dot{m}} \\
& \dot{W}_{d f}=\dot{m} \Delta h_{d f}
\end{aligned}
$$


where:

$\bar{\rho}$ denotes the average density between impeller inlet and outlet

$\mu_{2}$ denotes outlet viscosity

$f_{d f}$ denotes the friction factor

$\Delta h_{d f}$ denotes enthalpy rise due to disc friction

The value for disk friction power is then passed into Equation 3.61 for incorporation with the mixed-out state algorithm.

\subsubsection{Tip Leakage Loss}

The final external loss mechanism accounted for in the two-zone model analysis is tip leakage loss. Some fluid flow does not follow the passage exactly due to the necessity of maintaining a small clearance between the blade and the casing itself. Therefore, there is some small amount of fluid which does not experience compression within the impeller; the amount of flow that does not achieve compression compared with that if there was no clearance leads to reduction of efficiency.

Oh et al. (2002) recommends a model proposed by Aungier (2000), however due to the extra input required for this analysis, a more compact model developed by Jansen (1967) which has been used extensively in many compressor performance models is employed. This analysis requires no extra input of information while yielding acceptable 
results. Required information consists of: blade exit height, outlet absolute tangential velocity, number of impeller blades, inlet blade tip radius, outlet radius, inlet and outlet static densities and the inlet absolute axial velocity. The calculation proceeds as follows:

$$
\begin{gathered}
\varepsilon=0.01 b_{2} \\
\Delta h_{l k}=0.6 \frac{\varepsilon}{b_{2}} C_{\theta 2} \sqrt{\frac{4 \pi}{b_{2} Z} \frac{r_{1 t}^{2}-r_{1 h}^{2}}{\left(r_{2}-r_{1}\right)\left(1+\frac{\rho_{2}}{\rho_{1}}\right)} C_{\theta 2} C_{x 1}} \\
\dot{W}_{l k}=\dot{m} \Delta h_{l k}
\end{gathered}
$$

where:

$\varepsilon$ denotes clearance between the blade and the casing

$b_{2}$ denotes the exit blade height

$C_{\theta 2}$ denotes the impeller exit absolute tangential velocity

$C_{x l}$ denotes the inlet axial velocity

$\Delta h_{l k}$ denotes the enthalpy rise due to tip leakage

It is assumed here that the clearance is 1 percent of the outlet height, which is admittedly, very aggressive. According to Sjolander (2006), a clearance of 3 percent of the blade height is commonplace, however the range can be extended as low as 1 percent. Despite leaving the value at 1 percent, the user has the ability to change this to a value of their choice, depending on manufacturing parameters and any modelling constraints. The 
value for power increase due to tip leakage is then passed to the mixed-out state algorithm for use in Equation 3.61.

\subsubsection{Impeller Model Notes}

Equations 3.1 through 3.75 describe the complete impeller two-zone model. It became apparent after coding the impeller two-zone model, that the model had undocumented shortcomings. The two-zone model, as described above, has the tendency to generate non-sense data under certain conditions. In particular, the combination of low corrected mass flow rate and low corrected speed (less than 50 percent of the design rotational speed) seem to generate data which do not fit the trend expected of a compressor characteristic. Furthermore, very high corrected mass flow rates and high corrected rotational speeds (above 105 percent of the design speed) also tend to generate data which is of no use to the designer. It is for these reasons that when undertaking a new compressor design, it is advisable to keep the window of mass flow rates and speeds small at the beginning to generate confidence in the data.

Furthermore, the user is required to make decisions regarding what data is 'good data' and what is not. Since the impeller two-zone model outputs all data, good and bad to text files, user input is required to remove bad data and create new text files for each speed line, with the respective good data (including corrected mass flow rate, pressure ratio and efficiency). It is easy enough to input all data to Excel or a similar spreadsheet program and sort through the data. The format for creating text files for each speed line is as follows: the corrected speed value for the data must be placed on the first line. The 
remaining lines contain all 'good' data for that corrected speed line, specifically mass flow rate, pressure ratio and efficiency. Data for each mass flow rate must be contained the same row, as follows:

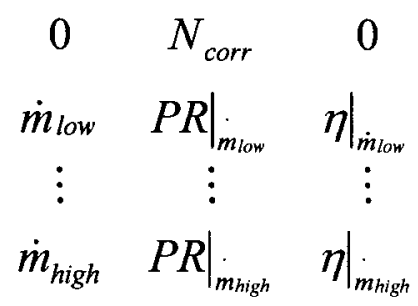

From this format, curve fits will be able to be applied to the data, to continue the calculations.

\subsection{Diffuser Performance Prediction}

As mentioned previously, the diffuser is an important component of any centrifugal compressor system, and as such, a model to incorporate pressure rise due to diffusion was necessary for completion. At present, a vaneless diffuser model is employed in conjunction with the impeller model, however it is hoped that future versions of the code will allow the user to employ a vaned diffuser as well.

The diffuser model which is used is adapted from Aungier (2000) and does not require any inputs other than the geometry of the diffuser itself and the flow conditions determined by the solution of the impeller model. The model involves the solution of continuity, angular momentum and linear momentum in a stepwise iterative scheme - the conditions at the first node are used to approximate the conditions at the second node, and 
so on, throughout the diffuser. External loss correlations are used to capture the diffusion losses as summarized below. The inputs to the model are: inlet diffuser radius $\left(r_{3}\right)$, outlet diffuser radius $\left(r_{4}\right)$, inlet diffuser passage width $\left(b_{3}\right)$, outlet diffuser passage width $\left(b_{4}\right)$, inlet absolute flow angle $\left(\alpha_{3}\right)$, inlet relative flow angle $\left(\beta_{3}\right)$, inlet stagnation temperature and pressure $\left(T_{03}\right.$ and $\left.P_{03}\right)$, inlet radial and tangential absolute velocities $\left(C_{m 3}\right.$ and $\left.C_{\theta 3}\right)$, mass flow rate $(\dot{m})$ and the number of solution nodes.

The main equations are continuity, conservation of angular momentum and conservation of linear momentum, as follows:

$$
\begin{gathered}
2 \pi r \rho b C_{m}(1-B)=\dot{m} \\
b C_{m} \frac{d\left(r C_{\theta}\right)}{d m}=-r C C_{\theta} c_{f} \\
\frac{1}{\rho} \frac{d P}{d m}=\frac{C_{\theta}}{r}-C_{m} \frac{d C_{m}}{d m}-\frac{C C_{m} c_{f}}{b}-\frac{d I_{D}}{d m}
\end{gathered}
$$

which, when cast into a finite difference scheme using Euler's method, become:

$$
\begin{aligned}
C_{m, i} & =\frac{\dot{m}}{2 \pi r_{i} \rho_{i} b_{i}\left(1-B_{i}\right)} \\
C_{\theta, i} & =\frac{r_{i-1} C_{\theta, i-1}}{r_{i-1}+\Delta m}-\frac{r_{i-1} C_{i-1} C_{\theta, i-1} c_{f_{i-1}} \Delta m}{b_{i-1} C_{m i-1}}
\end{aligned}
$$




$$
\begin{gathered}
P_{i}=P_{i-1}+\frac{\rho_{i-1} C_{\theta, i-1}^{2} \Delta m}{r_{i-1}}-\rho_{i-1} C_{m, i-1}\left(C_{m, i-1} C_{m, i-1}\right) \\
-\frac{\rho_{i-1} C_{i-1} C_{m, i-1} c_{f_{i-1}} \Delta m}{b_{i-1}}-\left.\rho_{i-1} \frac{d I_{D}}{d m}\right|_{i-1} \Delta m
\end{gathered}
$$

where:

$C_{m}$ denotes absolute meridional velocity

$r$ denotes radius within the vaneless diffuser

$B$ denotes blockage due to boundary layer growth (as evaluated in Equations 3.77

and 3.85)

$C_{\theta}$ denotes absolute tangential velocity

$\mathrm{C}$ denotes absolute velocity

$c_{f}$ denotes the skin friction coefficient

$\Delta m$ denotes the stepsize between nodes, in the meridional direction

$b$ denotes the vaneless diffuser width at the given node location

$\frac{d I_{D}}{d m}$ denotes diffusion loss in the vaneless diffuser

$i$ denotes the conditions at the location of the current node

$i-1$ denotes the conditions at the location of the previous node

The above equations are the main governing equations which need to be solved to determine diffuser exit conditions. However, the solution of the equations is slightly more complicated; the overall solution scheme is outlined as follows: 


$$
\begin{aligned}
& r C_{\theta e}=C_{\theta 3} r_{3} \\
& \delta_{3}=\frac{\left[1-\left(\frac{b_{3}}{r_{3}}\right)^{0.15}\right] b_{3}}{2} \\
& B_{3}=\frac{\delta_{3}}{4 b_{3}} \\
& c_{f}=0.02\left(\frac{\rho_{3} C_{3} \delta_{3}}{\mu_{3}}\right)^{-\frac{1}{6}} \\
& D_{m}=0.4\left(\frac{b_{3}}{r_{4}-r_{3}}\right)^{0.35} \sin \alpha_{2} \\
& C_{m, i-1}=\frac{\dot{m}}{2 \pi r_{i-1} \rho_{i-1} b_{i-1}\left(1-B_{i-1}\right)}
\end{aligned}
$$

where:

$$
\begin{aligned}
& r C_{\theta e} \text { denotes angular momentum at the inlet } \\
& \delta_{3} \text { denotes boundary layer thickness at the diffuser inlet } \\
& D_{m} \text { denotes a limiting value of the divergence parameter }
\end{aligned}
$$

Equations 3.83 and 3.86 are empirical expressions based upon many vaneless diffuser data sets. Specifically Equation 3.86 represents diffusion loss within the vaneless diffuser. The above outlines the diffuser inlet conditions and requires no iterative solution. This however, is where the loop begins, marching stepwise through the diffuser until the exit conditions have been determined. The number of nodes is user dependant, 
and should be increased until the results are grid independent. With the conditions at the first node known, the next node can be calculated:

$$
\begin{gathered}
C_{\theta, i}=\frac{r_{i-1} C_{\theta, i-1}}{r_{i-1}+\Delta m}-\frac{r_{i-1} C_{i-1} C_{\theta, i-1} c_{f_{i-1}} \Delta m}{b_{i-1} C_{m i-1}} \\
r_{i}=r_{i-1}+\Delta m \\
b_{i}=b_{3}+\frac{d b}{d r}\left(r_{i}-r_{3}\right) \\
\delta_{i}=2.25 b_{i}\left(1-\frac{r_{i} C_{\theta, i}}{r C_{\theta e}}\right) \\
B_{i}=\frac{\delta_{i}}{4 b_{i}} \\
C_{i}=\sqrt{C_{m, i-1}^{2}+C_{\theta, i}^{2}} \\
T_{i}=T_{03}-\frac{C_{i}^{2}}{2 c_{p}} \\
C_{m, i}=\frac{P_{i-1}}{R T_{i}} \\
2 \pi r_{i} \rho_{i} b_{i}\left(1-B_{i}\right) \\
\dot{m} \\
\rho_{i} \\
\end{gathered}
$$

Equation 3.91 denoting boundary layer thickness at the current node is another empirical expression developed with the analysis of many centrifugal compressor vaneless diffusers. Equations 3.93 through 3.96 are iterative and continue until the 
absolute meridional velocity has converged within a prescribed tolerance. The diffuser model continues by calculating the remaining quantities at the $\mathrm{i}^{\text {th }}$ node.

$$
\begin{aligned}
& D_{i}=-\frac{b_{i}}{C_{i}} \frac{C_{i}-C_{i-1}}{\Delta m} \\
& \text { if, } \\
& D_{i} \leq 0 \quad E_{i}=1 \\
& 0<D_{i}<D_{m} \quad E_{i}=1-0.2\left(\frac{D_{i}}{D_{m}}\right)^{2} \\
& D_{i} \geq D_{m} \quad E_{i}=0.8 \sqrt{\frac{D_{m}}{D_{i}}} \\
& \left.\frac{d I_{D}}{d m}\right|_{i-1}=-2\left(P_{03}-P_{i-1}\right)(1-E) \frac{1}{\rho_{i-1} C_{i-1}} \frac{C_{i}-C_{i-1}}{\Delta m} \\
& P_{i}=P_{i-1}+\frac{\rho_{i-1} C_{\theta, i-1}^{2} \Delta m}{r_{i-1}}-\rho_{i-1} C_{m, i-1}\left(C_{m, i-1} C_{m, i-1}\right) \\
& -\frac{\rho_{i-1} C_{i-1} C_{m, i-1} c_{f_{i-1}} \Delta m}{b_{i-1}}-\left.\rho_{i-1} \frac{d I_{D}}{d m}\right|_{i-1} \Delta m \\
& c_{f i}=0.02\left(\frac{\rho_{i} C_{i} \delta_{i}}{\mu_{i}}\right)^{-\frac{1}{6}}
\end{aligned}
$$

where:

$D_{i}$ denotes the divergence parameter at the current node E denotes the an empirical diffusion efficiency 
$\mu$ denotes viscosity at the current node

Equations 3.97 through 3.99 are also empirical expressions based upon vaneless diffuser data sets, as outlined by Aungier (2000) and like Equation 3.86, represent diffusion loss. Equations 3.88 through 3.101 are iterative throughout the diffuser, marching from inlet to outlet. Once the solution scheme reaches the final node, the outlet conditions are known. The above solution scheme works for one mass flow rate and must be repeated for each combination of mass flow rate and rotational speed. For ease, it is recommended that the above model be employed within the two-zone model as a subprogram.

\subsection{Low Speed Extrapolation Algorithm}

To complete the compressor map, the low speed area must be determined. Although not documented in the literature, the solution of the two-zone model appears to converge well between the range of 50 percent to 105 percent of the design speed. These values are merely guidelines as determined through the operation of the two-zone model and should not be taken as hard limits of the two-zone model. As such, a method to determine the lower speed region is required. Gaudet and Gauthier (2007) proposed a simple, sub-idle extrapolation technique, which is applicable to the problem at hand and is outlined below. 
To complete the analysis, several parameters are required for the module, specifically: the data from the two lowest speed characteristics (corrected mass flow rate, pressure ratio and efficiency), the inlet pressure and temperature and the design speed of the compressor. Furthermore, the establishment of a beta grid is required for the analysis to continue. Beta grid definition will be described in section 3.4.

The method developed by Gaudet and Gauthier is an updated model of the well known similarity expressions developed by Sexton (2001). The updated similarity expressions are as follows:

$$
\begin{aligned}
& \left(\frac{\dot{m} \sqrt{T_{01}}}{P_{01}}\right)_{a} \\
& \left.\frac{\dot{m} \sqrt{T_{01}}}{P_{01}}\right)_{b} \\
& \left.\frac{N_{b}}{N_{a}}\right]^{p} \\
& \frac{\boldsymbol{w}_{\text {ideal }_{b}}}{\boldsymbol{w}_{\text {ideal }, a}}=\left[\frac{N_{b}}{N_{a}}\right]^{a} \\
& \frac{\dot{W_{b}}}{\dot{W}_{a}}=\left[\frac{N_{b}}{N_{a}}\right]^{r}
\end{aligned}
$$

where,

$$
N=\frac{N / \sqrt{T_{01}}}{N_{d e s} / \sqrt{T_{01}}}
$$

The above variables are defined as follows;

$a$ denotes the quantities of reference speed line a 
$b$ denotes the quantities of reference speed line $b$

$N$ denotes rotational speed in RPM

$w$ denotes the specific work

$\dot{W}$ denotes power

$p, q$ and $r$ denote the similarity exponents

From these expressions, it is possible to rearrange the equations to solve for the similarity exponents p, q and r. By rearranging Equation 3.102, an expression for $\mathrm{p}$ can be obtained:

$$
p=\frac{\log \left[\frac{\left(m \sqrt{T_{01}} / P_{01}\right)_{b}}{\left(m \sqrt{T_{01}} / P_{01}\right)_{a}}\right]}{\log \left(\frac{N_{b}}{N_{a}}\right)}
$$

An expression for $\mathrm{q}$ is obtained by incorporating the expression for ideal specific work. From first principles, the ideal specific work across the compressor is:

$$
w_{\text {ideal }}=c_{p} T_{01}\left[(P R)^{\frac{\gamma-1}{\gamma}}-1\right]
$$

Equation 3.107 can be substituted into Equation 3.103 which can be rearranged to determine the similarity exponent, q: 


$$
q=\frac{\log \left[\frac{\left(P R^{\frac{\gamma-1}{\gamma}}-1\right)_{b}}{\left.\left(P R^{\frac{\gamma-1}{\gamma}}-1\right)_{a}\right]}\right.}{\log \left[\frac{N_{b}}{N_{a}}\right]}
$$

Similarly, an expression for $\mathrm{r}$ can be obtained by incorporating an expression for the power requirement, $\dot{W}$ :

$$
\dot{W}=\dot{m} c_{p} \frac{T_{01}}{\eta}\left[(P R)^{\frac{\gamma-1}{\gamma}}-1\right]
$$

Equation 3.109 can be substituted into Equation 3.104 and rearranged, along with the expressions for $p$ and $q$, to obtain:

$$
r=(p+q)-\frac{\log \left(\frac{\eta_{b}}{\eta_{a}}\right)}{\log \left(\frac{N_{b}}{N_{a}}\right)}
$$

By determining the similarity exponents $\mathrm{p}, \mathrm{q}$ and $\mathrm{r}$, one can then obtain the pressure ratio, mass flow rates and efficiency at any speed line desired through the relationships below: 


$$
\begin{aligned}
& P R_{x}=\left[1+\left(\left(P R_{a}\right)^{\frac{\gamma-1}{\gamma}}-1\right)\left(\frac{N_{x}}{N_{a}}\right)^{q}\right]^{\frac{\gamma}{\gamma-1}} \\
& \left(\frac{m \sqrt{T_{01}}}{P_{01}}\right)_{x}=\left(\frac{m \sqrt{T_{01}}}{P_{01}}\right)\left[\frac{N_{x}}{N_{a}}\right]^{p} \\
& \eta_{x}=\eta_{a}\left[\frac{N_{x}}{N_{a}}\right]
\end{aligned}
$$

where:

$x$ denotes the value of a quantity for the desired speed line

Similarity exponents are calculated for each beta line in the beta grid using the two bottommost speed characteristics. Using the similarity exponents, as a function of beta value, one can obtain the pressure ratio, mass flow rate and efficiency for any value of rotational speed.

\subsection{Beta Grid Definition Algorithm}

The use of a beta line grid has been well documented for use in map scaling techniques, specifically by Kurzke (1996). As shown by Kurzke, it is difficult to use a compressor map in the standard format for performance calculations. Due to the fact that constant speed characteristics can become vertical on the compressor map, indicating choking of flow, and also that there can be two values of corrected mass flow rate for a given pressure ratio (due to the curvature of the constant speed characteristics), auxiliary 
coordinates can be introduced which intersect with the speed lines to aid with several calculations. These lines are termed $\beta$-lines. Figure 3.6 shows a typical beta grid for a compressor map.

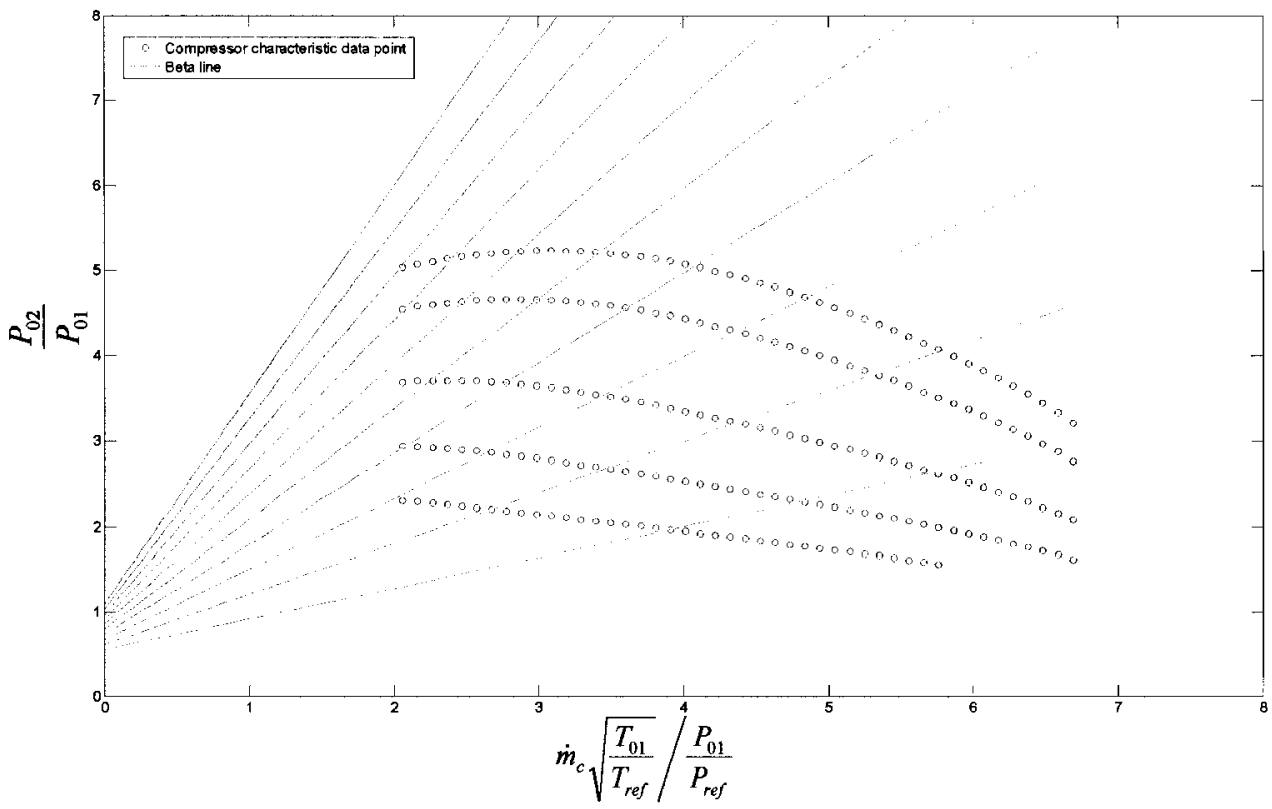

Figure 3.6 Typical beta line grid for use in association with compressor maps.

Parabolas are usually used as the shape for the beta lines, however straight lines can be used if desired. Kurzke gives guidelines for establishing the limiting beta lines. Kurzke recommends upper beta line $(\beta=1)$ pass through the point of zero corrected mass flow rate, and a pressure ratio of one. The lower beta line $(\beta=0)$ should be roughly parallel to the upper beta line, to make sure they do not cross one another in the range of interest.

An algorithm has been developed to define the beta grid for a given compressor map. The user must supply a text file of the raw data developed by the two-zone model. The text file must be formatted in a specific manner for the algorithm to assimilate the 
data. A zero must be the first value on the first line of the text file, followed by a tabbed space where the lowest corrected speed value is given. Following these two values, all the values for corrected mass flow are supplied in the left column followed by a tabbed space with the pressure ratio for that given corrected speed value. Once all the data for a given speed line had been supplied, another zero is supplied, followed by a tabbed space then the second value for corrected speed. Data for corrected mass flow rate and pressure ratio for the second corrected speed value are then supplied. This format continues until all data for the compressor map have been entered. An example of a formatted beta line input file is included in Appendix C.

Once the algorithm is started, the user is asked to supply the text file as well as the number of beta lines to define. An increased number of beta lines increases the smoothness of the extrapolated speed lines, however increases the computation time.

It was determined during coding that using a parabola would not properly capture the data and it was decided that straight lines could more easily capture the data sets which were generated by the two-zone model. The algorithm proceeds in a fashion similar to that outlined by Kurzke (1996). Figure 3.7 shows a flow chart of the algorithm operation. 


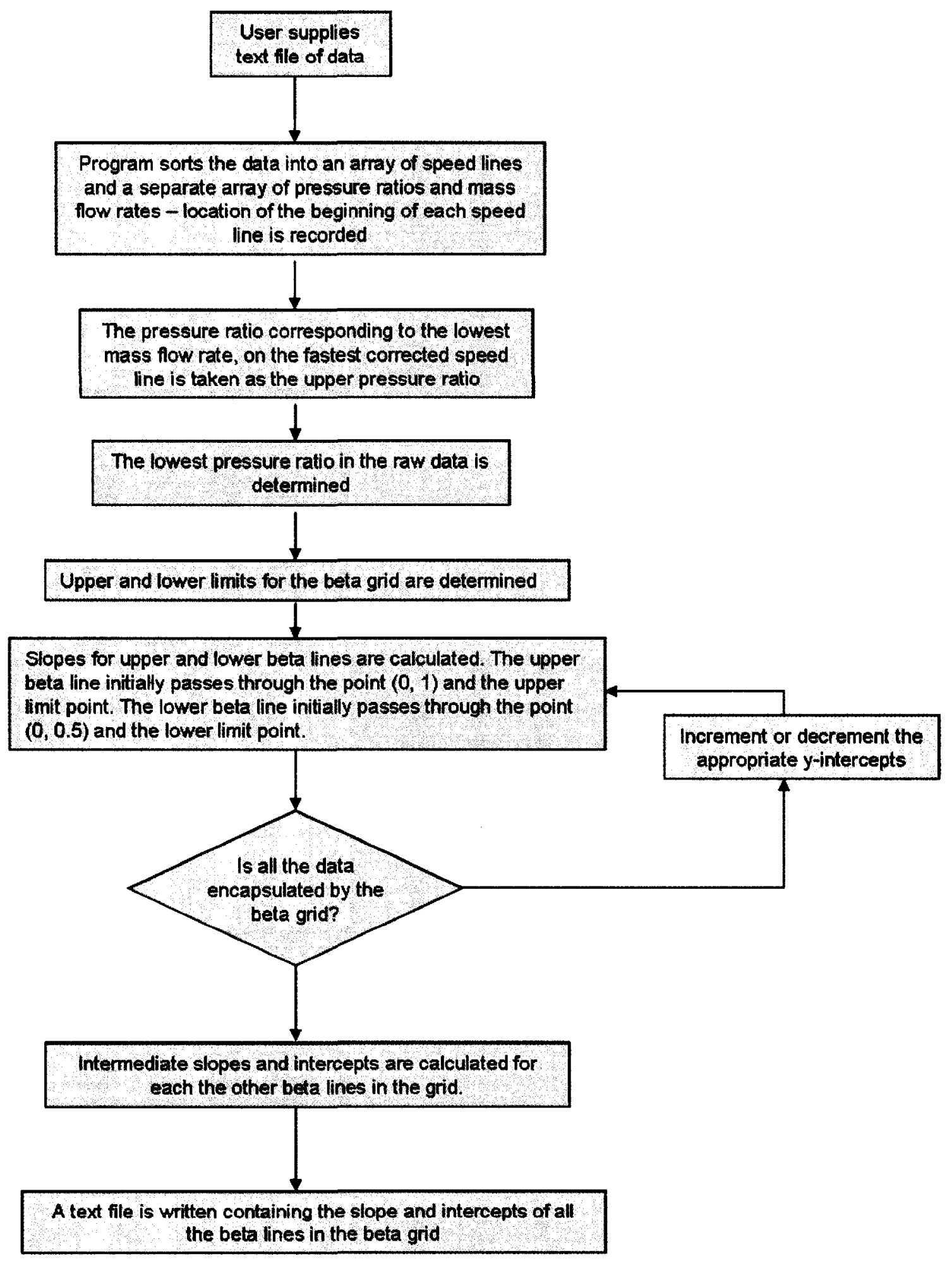

Figure 3.7 Beta line algorithm as used in conjunction with the off-design performance code. 


\subsection{Supporting Codes}

To complete the overall performance prediction program, some supporting modules must be included to satisfy operation. Specifically the addition of a curve fitting algorithm and a Newton-Raphson solver are needed.

\subsubsection{Curve Fitting Algorithm}

A curve fitting algorithm is needed to complete the off-design analysis due to the inherent calculations of the impeller model and the beta grid definition model. Both algorithms generate discrete data points, not continuous functions. Furthermore, the low speed extrapolation model requires specific intersection points of the two lowest speed lines with all the generated beta lines ( 0 though 1$)$. From examination of any generated data, it became clear that it was very unlikely that the beta lines would be calculated at precisely the same location as a generated compressor map point. Therefore, to satisfy the problem, continuous functions must be obtained to determine the location of intersection.

Once the impeller data has been sorted through by the user to determine its validity, the user then can supply the data to the next component of the gas turbine performance modeling algorithm. Once the curve fitting algorithm is started, the user is asked to supply the number of text files corresponding to the number of speed lines. Once this is done, the user is then asked to enter the names of all the text files containing the good data. The data for each speed line is then fit to a polynomial of degree four by the curve fitting algorithm supplied in MATLAB. The data containing the coefficients for 
each line of pressure ratio and efficiency, and the range of mass flow rates over which the polynomial fit is valid is then written to a text file for further use.

\subsubsection{Newton-Raphson Solver}

To determine the intersection of the speed characteristics with the beta lines, a numerical solver needed to be coded. Several alternatives for solving the system of nonlinear equations including: secant method, bisection method and perhaps the best known, the Newton-Raphson method. To determine the locations of intersection, the NewtonRaphson solver needs to be supplied with the coefficients for the $4^{\text {th }}$ order curve fit to

pressure ratio data $\left(f_{1}(x)\right)$ as well as the details of each beta line $\left(f_{2}(x)\right)$ (specifically slope and intercept).

The Newton-Raphson solver determines the location of the intersection as follows. At intersection, $f_{1}(x)=f_{2}(x)$; therefore $f_{3}(x)=f_{1}(x)-f_{2}(x)=0$, where:

$$
\begin{aligned}
& f_{1}(x)=a_{0} x^{4}+a_{1} x^{3}+a_{2} x^{2}+a_{3} x+a_{4} \\
& f_{2}(x)=a_{5} x+a_{6}
\end{aligned}
$$

where, $a_{0}$ to $a_{4}$ correspond to the coefficient developed by the least squares fit of the good data and, $a_{5}$ and $a_{6}$ correspond to the slope and y-intercept of the beta lines, respectively. 
Therefore, to determine the location of intersection, one needs to determine the roots of the equation $f_{3}(x)$.

An arbitrarily large guess for the x-location of intersection is supplied, $x_{\text {guess }}$, to the algorithm to avoid finding any negative roots. An approximation to the root of the equation is then determined by the equation:

$$
x_{\text {new }}=x_{\text {guess }}-\frac{f_{3}\left(x_{\text {guess }}\right)}{f_{3}^{\prime}\left(x_{\text {guess }}\right)}
$$

The value for $x_{\text {guess }}$ is then replaced by the value for $x_{\text {new }}$ and another approximation to the root is obtained. This continues until the difference between $x_{\text {guess }}$ and $x_{\text {new }}$ is very small and can be considered to have converged.

The values for mass flow rate, pressure ratio and efficiency are recorded at each intersection point of the speed characteristic and each beta line and passed to the algorithm for low speed extrapolation.

\subsection{Component Matching Algorithm}

Validation of the compressor off-design model requires the ability to perform full engine off-design analysis, including the estimation of the equilibrium running line and subsequently calculated parameters such as specific fuel consumption, power 
requirements and rotational speed plots. Engine off-design analysis requires off-design information of the compressor and the turbine used as well as other known design parameters including combustor pressure losses and any air bleeds in the engine. Therefore, to use the compressor map generated by the above model, a turbine map must also be developed.

\subsubsection{Turbine Map Generation}

Turbine off-design analysis was not the focus of the thesis and as such, simple means of generating turbine maps were deemed acceptable. A design point matching technique was used, similar to that described for compressors in the literature review. Turbine maps obtained in open literature were normalized with respect to the design pressure ratio and design mass flow rate and then digitized using a plot digitizing software package. The normalized turbine pressure ratio and efficiency characteristics can be seen in Figures 3.8 and 3.9, respectively. 


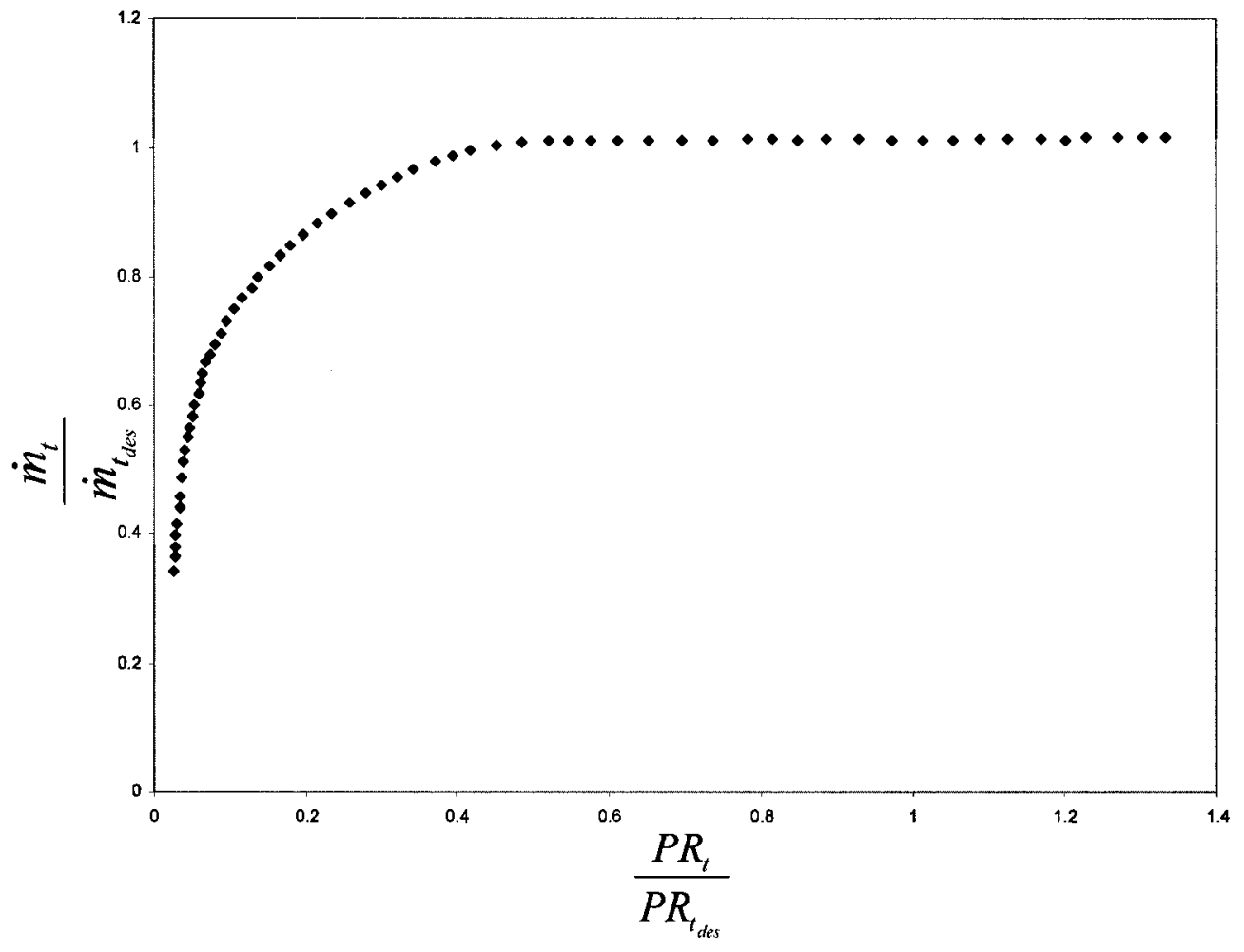

Figure 3.8 Normalized turbine characteristic used in the turbine map scaling process.

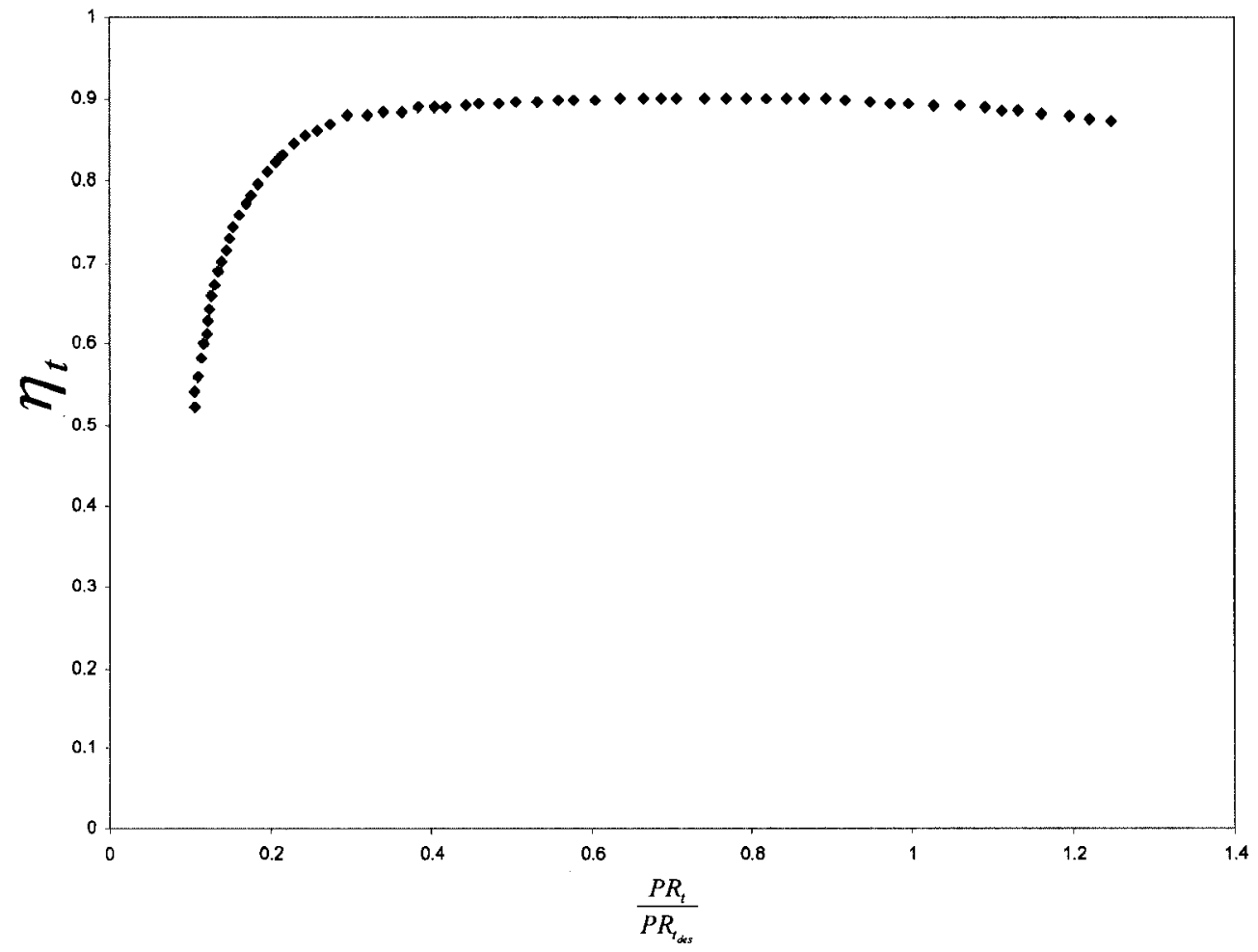

Figure 3.9 Normalized efficiency characteristic used in the turbine map scaling process. 
Non-normalized characteristics are then obtained by multiplying each point on the pressure ratio axis (abscissa on Figure 3.8) by the design point pressure ratio, and each point on the y-axis by the design point corrected mass flow rate. Similarly, the efficiency can be determined by multiplying each point on the pressure ratio axis (abscissa of Figure 3.9) by the design point pressure ratio and each point on the y-axis by the design point turbine isentropic efficiency:

$$
\begin{aligned}
& \left.\frac{\dot{m} \sqrt{T_{03}}}{P_{03}}\right|_{\text {non-nomalized }}=\left.\left(\frac{m \sqrt{T_{03}}}{P_{03}}\right)_{\text {des }} \frac{m \sqrt{T_{03}}}{P_{03}}\right|_{\text {normalized }} \\
& P R_{\text {non-nomalized }}=\left[\left(P R_{\text {normalized }}\right)\left(P R_{\text {des }}-1\right)+1\right]
\end{aligned}
$$

Once the new map quantities are determined, each of the characteristics can be curve fit and written to an ASCII file for use in conjunction with the generated compressor map.

\subsubsection{Equilibrium Running Line Determination}

With the compressor and turbine maps established, the equilibrium running line can be calculated. The following steps are taken to calculate the running line:

1. Design point performance is established:

a. Turbine pressure ratio is calculated, assuming a 3 percent pressure loss across the combustion chamber: 


$$
P R_{t}=0.97 P R_{c}
$$

b. Corrected mass flow rate and turbine isentropic efficiency is read from the turbine characteristic.

c. Compressor work, turbine work and net work are calculated at the design point:

$$
\begin{aligned}
& \dot{W}_{c}=\frac{\dot{m} C_{p} T_{01}}{\eta_{c}}\left[P R_{c}^{\frac{\gamma-1}{\gamma}}-1\right] \\
& \dot{W}_{t}=\dot{m} C_{p} T_{03} \eta_{t}\left[1-\left(\frac{1}{P R_{t}}\right)^{\frac{\gamma-1}{\gamma}}\right] \\
& \dot{W}_{n e t}=\dot{W}_{t}-\dot{W}_{c}
\end{aligned}
$$

d. Calculate work proportionality constant for the cube power relationship from Equation 2.18:

$$
k_{1}=\frac{\dot{W}_{\text {neldes }}}{N_{\text {des }}^{3}}
$$

Part-load net work output is then determined through the use of the cube power relationship at off-design speeds.

2. Start with the lowest speed characteristic

3. Calculate work requirement at the speed using cube power relationship:

$$
\dot{W}_{n e t_{\text {req }}}=k_{1} N^{3}
$$

4. Guess value for corrected mass flow on the compressor characteristics; read the value for compressor pressure ratio and efficiency from the map.

5. Calculate turbine pressure ratio using Equation 3.119. 
6. Read the corrected mass flow rate and isentropic efficiency of the turbine characteristics.

7. Calculate the turbine inlet temperature by flow compatibility:

$$
T_{03}=T_{\text {ref }}\left(\frac{\dot{m}_{c o r_{t}}}{\dot{m}} \frac{P_{03}}{P_{0 r e f}}\right)^{2}
$$

8. Calculate compressor work, turbine work and net work output using Equations 3.120 to 3.122 .

9. Net work output for the speed line will not, in general, agree with the net work requirement calculated in Equation 3.124. The guessed value for compressor corrected mass flow is altered and steps 4 through 9 are repeated until the net work requirement is met - this is the equilibrium point for that speed characteristic. If the net work requirement cannot be met, that speed line is not a valid operating speed and algorithm proceeds with step 10.

10. The analysis proceeds to the next speed line and steps 3 through 9 are repeated.

11. Net work, mass flow rate, compressor pressure ratio, compressor isentropic efficiency, turbine inlet temperature and compressor discharge temperature are recorded at each equilibrium point for use in any performance calculations.

Steps 1 through 11 outline the necessary steps to determine the equilibrium running line assuming the compressor and turbine maps have been established. 


\subsection{Performance Calculations}

With the running line determined, several important performance quantities can be determined along the running line itself. Several important performance plots are outlined in Section 2.2, those being:

- Rotational speed vs. turbine inlet temperature

- Net work output vs. turbine inlet temperature

- Net work output vs. specific fuel consumption

Of the three mentioned, two can be determined from the evaluation of the running line (rotational speed vs. turbine inlet temperature and net work output vs. turbine inlet temperature). Turbine inlet temperature, net work output, compressor discharge temperature, pressure ratio and mass flow rate as a function of rotational speed have been calculated by determination of the running line. One of the more important indications of gas turbine performance and efficiency is the variation of specific fuel consumption with net work output.

To determine the specific fuel consumption, one must determine the fuel-to-air ratio at that particular operating point. This requires specification of a heating value for the fuel which is defined of heat (or energy) released during the combustion of a specified amount of fuel. It is assumed that the gas used in the gas turbine engine is methane, however this can be altered during coding of the algorithm, or allowing for a user defined heating value. The fuel-to-air ratio can be calculated as: 


$$
\frac{f}{a}=\frac{h\left(T_{03}\right)-h\left(T_{02}\right)}{\left(\eta_{\text {comb }} H H V\right)-h\left(T_{01}\right)}
$$

Where $\eta_{\text {comb }}$ represents the combustion efficiency (assumed constant in this analysis at 99 percent) and HHV represented the high heating value of the fuel $(55.528 \mathrm{MJ} / \mathrm{kg}$ for methane from Turns (2000)).

The mass flow rate of fuel required for the fuel-to-air ratio calculated by Equation 3.126 for the given operating point can be determined by:

$$
\dot{m}_{\text {fuel }}=\dot{m}_{\text {air }} \frac{f}{a}
$$

where:

$$
\begin{aligned}
& \dot{m}_{\text {fuel }} \text { denotes the estimated fuel flow rate } \\
& \dot{m}_{\text {air }} \text { denotes the mass flow rate of air through the gas turbine engine } \\
& \frac{f}{a} \text { denotes the fuel-to-air ratio }
\end{aligned}
$$

Once the mass flow rate of fuel for the given operating point is calculated, the specific fuel consumption can be established:

$$
S F C=\frac{\dot{m}_{\text {fuel }}}{\dot{W}_{n e t}}
$$


An overall algorithm has been proposed to determine the impeller map with associated vaneless diffuser, a beta line grid development technique, a lower speed extrapolation method and the component matching algorithm.

The following chapter will outline the verification of the model against theory through the utilization of several resources including compressor maps available in the literature as well as CFD techniques. 


\section{Validation and Results}

A full set of equations modelling impeller off-design behaviour has been presented along with other necessary models to construct a full centrifugal compressor map for the off-design analysis of gas turbine engines. The scope of this chapter is to compare the results generated by the aforementioned model with actual impeller maps available in the literature with the ultimate goal of validating the proposed model for use in other gas turbine engine design scenarios.

The following chapter outlines the methodology and presents the results generated by centrifugal impeller performance prediction software package. This is split into four subsections: 1) validation of the impeller model, 2) validation of the low speed extrapolation model, 3) validation of the performance modelling algorithm and finally 4) performance modelling calculations. Geometries of four impellers have been obtained from the literature for use in the validation process encompassing a wide range of mass flow rate ranges and rotational speeds as well as design pressure ratios.

\subsection{Test Impellers}

The four impellers were obtained from the literature, two of which have been extremely well documented and used in the validation of other two-zone models, the Eckhardt impellers (Eckhardt, 1980) which have low design point pressure ratios and low 
design mass flow rates. To gain confidence in the model, higher pressure ratio impellers were required for validation at varying design conditions. The impeller described by Came and Herbert (1980) fits this criterion with a design pressure ratio of 8:1. Finally, an intermediate pressure ratio impeller was obtained from the geometry described by McKain and Holbrook (1997). Table 4.1 shows the design point performance, pertinent impeller geometry as well as any values required as input to the two-zone model.

Table 4.1 Specifications of test impeller used in two-zone model validation.

\begin{tabular}{|c|c|c|c|c|}
\hline (2) & $\begin{array}{c}\text { Eckhardt O } \\
\text { (Eckhardt, } \\
\text { 1980) }\end{array}$ & $\begin{array}{c}\text { Eckhardt A } \\
\text { (Eckhardt, } \\
1980 \text { ) }\end{array}$ & $\begin{array}{c}\text { Came and } \\
\text { Herbert (1980) }\end{array}$ & $\begin{array}{c}\text { McKain and } \\
\text { Holbrook } \\
\text { (1997) }\end{array}$ \\
\hline $\mathrm{P}_{01}[\mathrm{kPa}]$ & 101.3 & 101.3 & 101.3 & 101.3 \\
\hline $\mathrm{T}_{01}[\mathrm{~K}]$ & 288 & 288 & 288 & 305 \\
\hline $\mathrm{N}_{\mathrm{des}}[\mathrm{RPM}]$ & 14,000 & 14,000 & 40,000 & 21,800 \\
\hline$\dot{m}_{d e s}[\mathrm{~kg} / \mathrm{s}]$ & 5.32 & 4.5 & 1.81 & 4.7 \\
\hline $\mathrm{PR}_{\text {des }}$ & 2.2 & 1.9 & 8 & 4 \\
\hline $\mathrm{r}_{\mathrm{lh}}[\mathrm{mm}]$ & 45 & 60 & 30.48 & 41.37 \\
\hline$r_{11}[\mathrm{~mm}]$ & 140 & 140 & 67.3 & 104.95 \\
\hline $\mathrm{r}_{2}[\mathrm{~mm}]$ & 200 & 200 & 137.4 & 215.2 \\
\hline$b_{1}[\mathrm{~mm}]$ & 95 & 80 & 36.82 & 63.58 \\
\hline$b_{2}[\mathrm{~mm}]$ & 26 & 26 & 5.81 & 17.05 \\
\hline$\beta_{2 b}$ & 0 & 30 & 30 & 50 \\
\hline$Z_{\text {fill }}$ & 20 & 20 & 17 & 15 \\
\hline$Z_{\text {split }}$ & 0 & 0 & 17 & 15 \\
\hline $\mathrm{t}_{2 \mathrm{~b}}[\mathrm{~mm}]$ & 3 & 3 & 3 & 3 \\
\hline$\beta_{1 h}$ & 63 & 63 & 31.36 & 45 \\
\hline$\beta_{1 t}$ & 63 & 63 & 53.81 & 45 \\
\hline$\eta_{a}$ & 0.7 & 0.83 & 1.05 & 0.9 \\
\hline$\eta_{b}$ & 0.482 & 0.45 & 0.30 & 0.6 \\
\hline$\chi$ & 0.15 & 0.15 & 0.15 & 0.15 \\
\hline
\end{tabular}

For the two Eckhardt impellers, vaneless diffuser geometry was available and was used in the two-zone modelling validation, however both the higher pressure ratio impellers had no vaneless diffuser data, or vaned diffuser data associated with them; 
therefore, only the Eckhardt impellers employed the vaneless diffuser algorithm outlined in the proposed model. The vaneless diffuser for the Eckhardt impellers had an outlet radius of $0.4 \mathrm{~m}$ while maintaining the flow area.

\subsection{Two-Zone Model Validation}

Impeller maps were generated by the two-zone model for comparison against actual maps. For each speed line on each map, the pressure ratio and isentropic efficiency was determined by the in-house code and compared against the actual map values digitized from the literature. Plots showing pressure ratio, isentropic efficiency and difference between the predicted value by in-house and the actual value from the impeller map are shown below. Discrete data points represent the digitize map value, while the solid lines represent the corresponding values determined by the two-zone model.

Figures 4.1 through 4.8 show the performance of two impellers described by Eckhardt (1980) designated as O for the radial impeller and A for the backswept impeller. Both impellers have very low design point pressure ratios as indicated in Table 4.1. Figures $4.1,4.2,4.5$ and 4.6 show the characteristics of both the actual map obtained from the literature as compared with the compressor map as determined by the two-zone model (TZM). Figures 4.3, 4.4, 4.7 and 4.8 show the difference between the actual map value and the map value determined by the two-zone model (TZM) for both pressure ratio and isentropic efficiency. Figures 4.1 through 4.4 correspond to Eckhardt impeller $\mathrm{O}$ while figures 4.5 through 4.8 correspond to Eckhardt impeller A. 


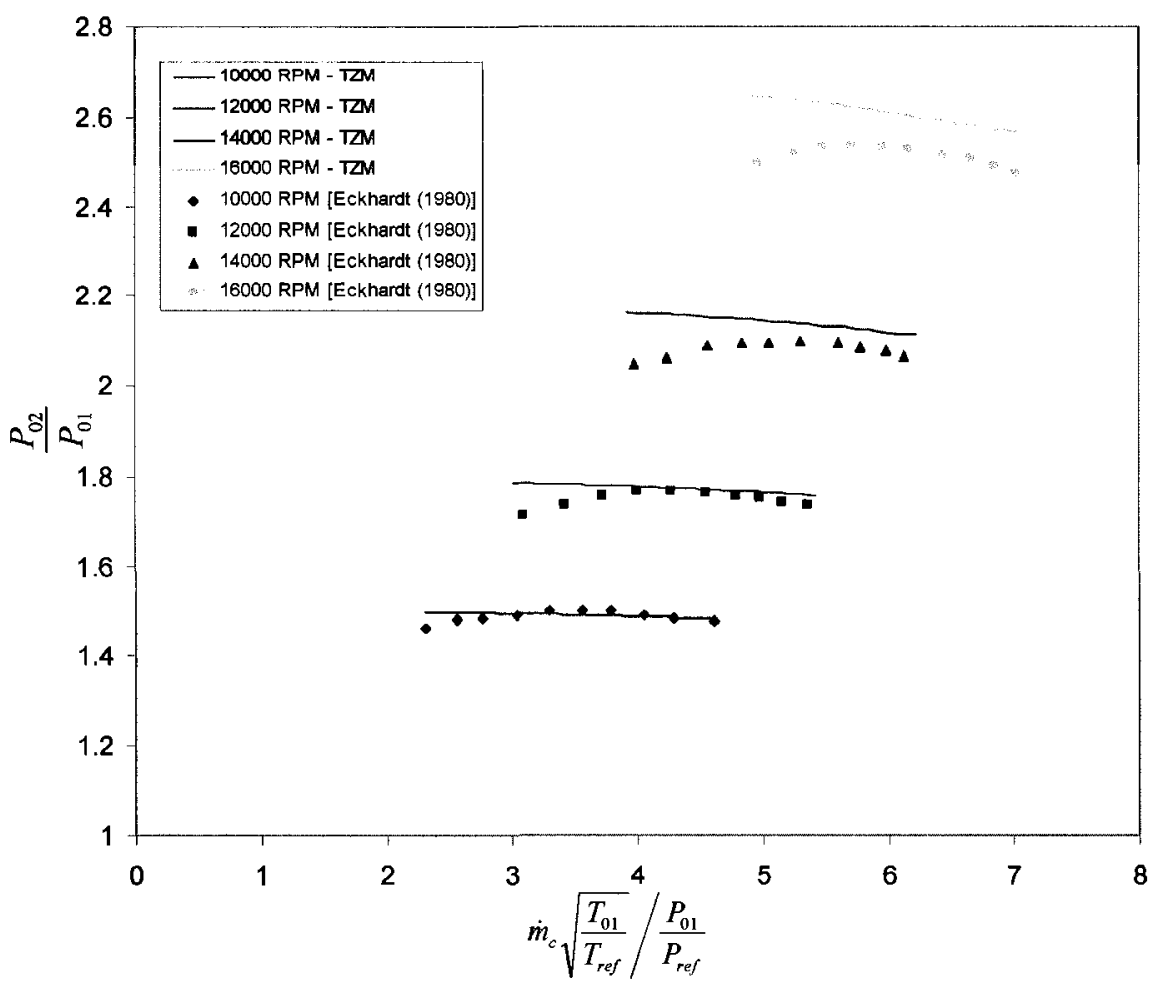

Figure 4.1 Pressure ratio of Eckhardt impeller O.

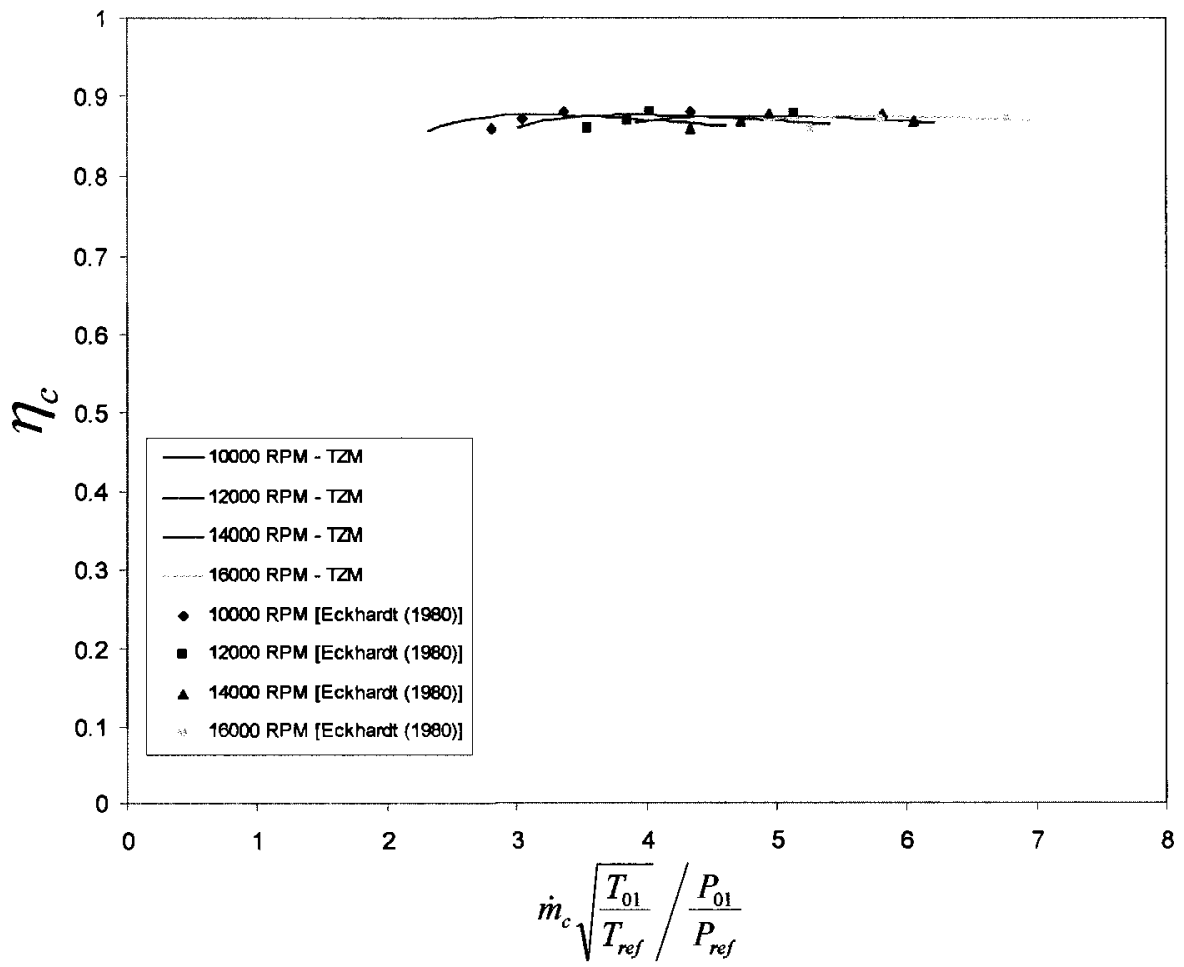

Figure 4.2 Isentropic efficiency of Eckhardt impeller O. 


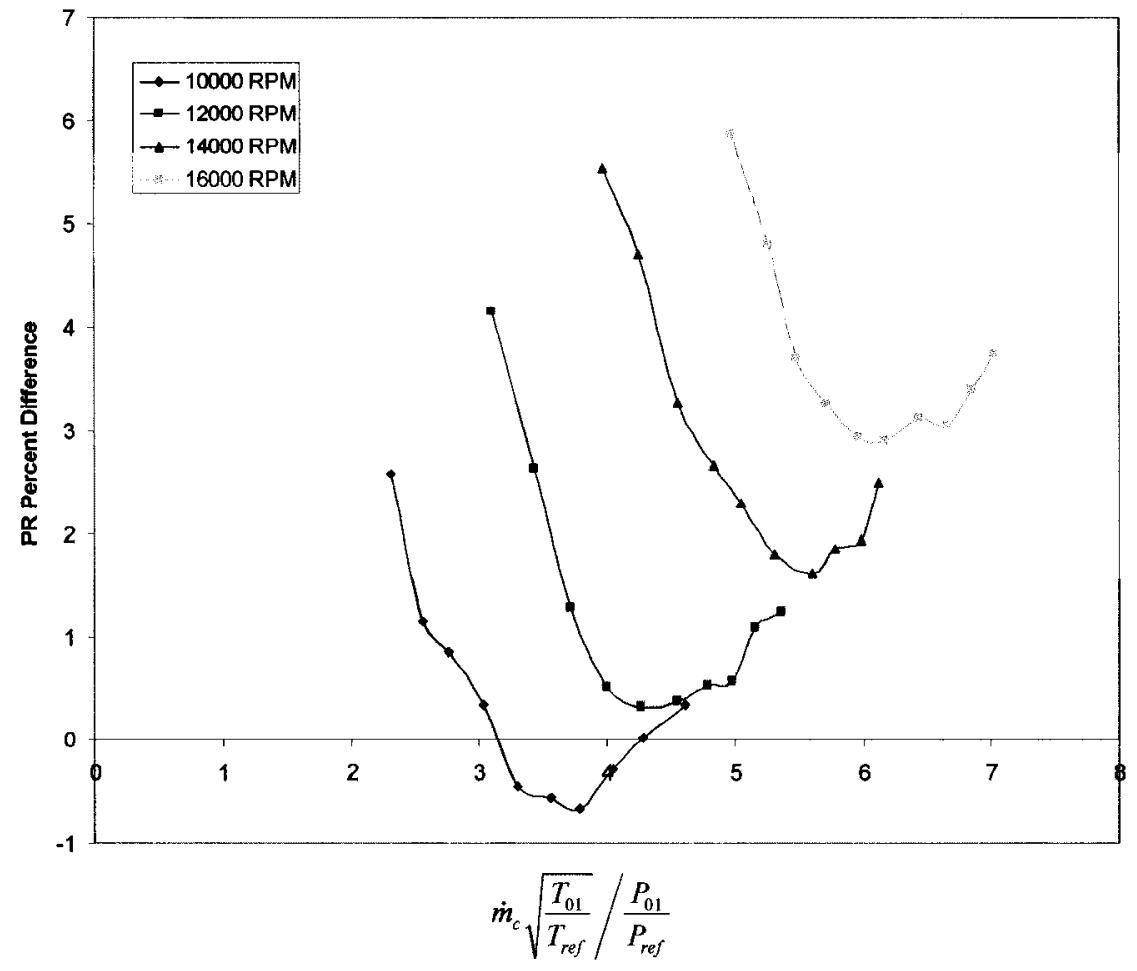

Figure 4.3 Difference between pressure ratio generated by TZM and the value from the actual impeller map for Eckhardt impeller $O$.

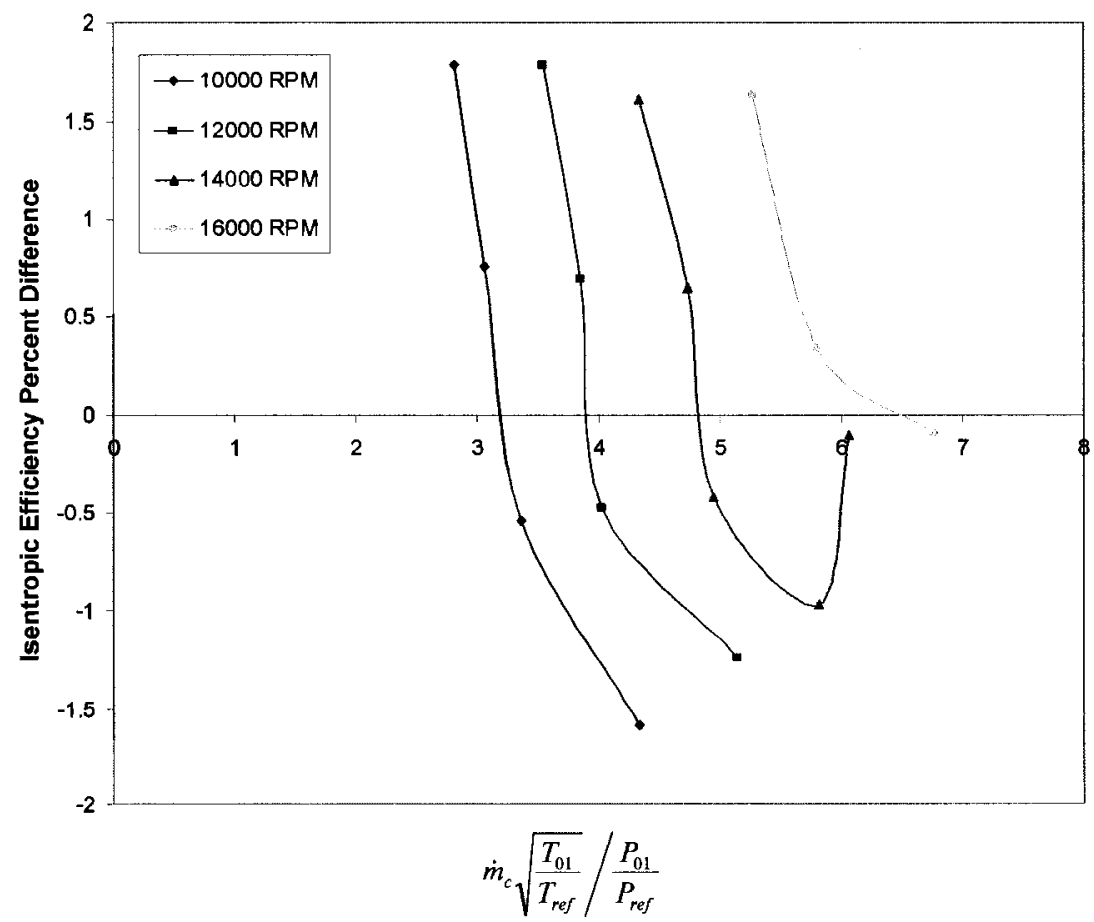

Figure 4.4 Difference between isentropic efficiency generated by TZM and the value from the actual impeller map for Eckhardt impeller O. 
Figures 4.1 through 4.4 indicate the two-zone model accurately describes the Eckhardt $\mathrm{O}$ impeller over the range of mass flow rates indicated by the actual map, as well the range of rotational speeds indicated by the actual map. General trends indicate poorer estimation of the impeller map as the rotational speed increases, however the difference between the actual map pressure ratio and the pressure ratio generated by the two-zone model is limited within 6 percent. Furthermore, the isentropic efficiency indicates even better agreement with the actual map values, staying within 2 percent difference over the whole range of rotational speeds and mass flow rates.

Subsequent testing of Eckhardt impeller $\mathrm{O}$ with the two-zone model code indicates that the model will generate data outside what was presented by Oh et al. (2002) and initially by Eckhardt (1980). The limits on convergence for impeller O lie between 1 and $7 \mathrm{~kg} / \mathrm{s}$ for corrected mass flow and also between 50 percent and 115 percent of design speed.

The following four figures show the performance for the impeller designated Eckhardt impeller A. Figures 4.5 and 4.6 indicate the pressure ratio and efficiency of the actual map value compared with that determined by the two-zone model (TZM). Figures 4.7 and 4.8 show the difference of pressure ratio and efficiency between the actual map value and the value determined by the two-zone model (TZM). 


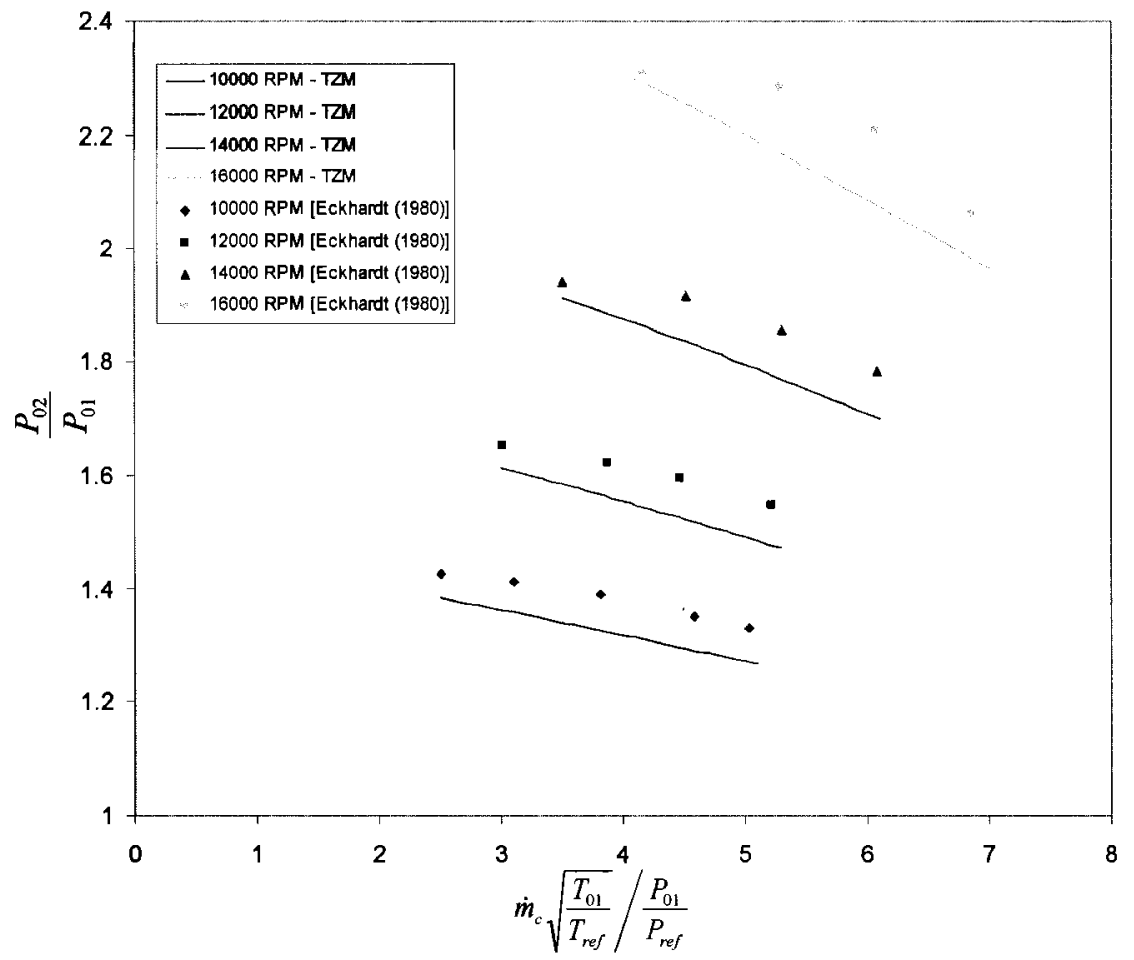

Figure 4.5 Pressure ratio of Eckhardt impeller A.

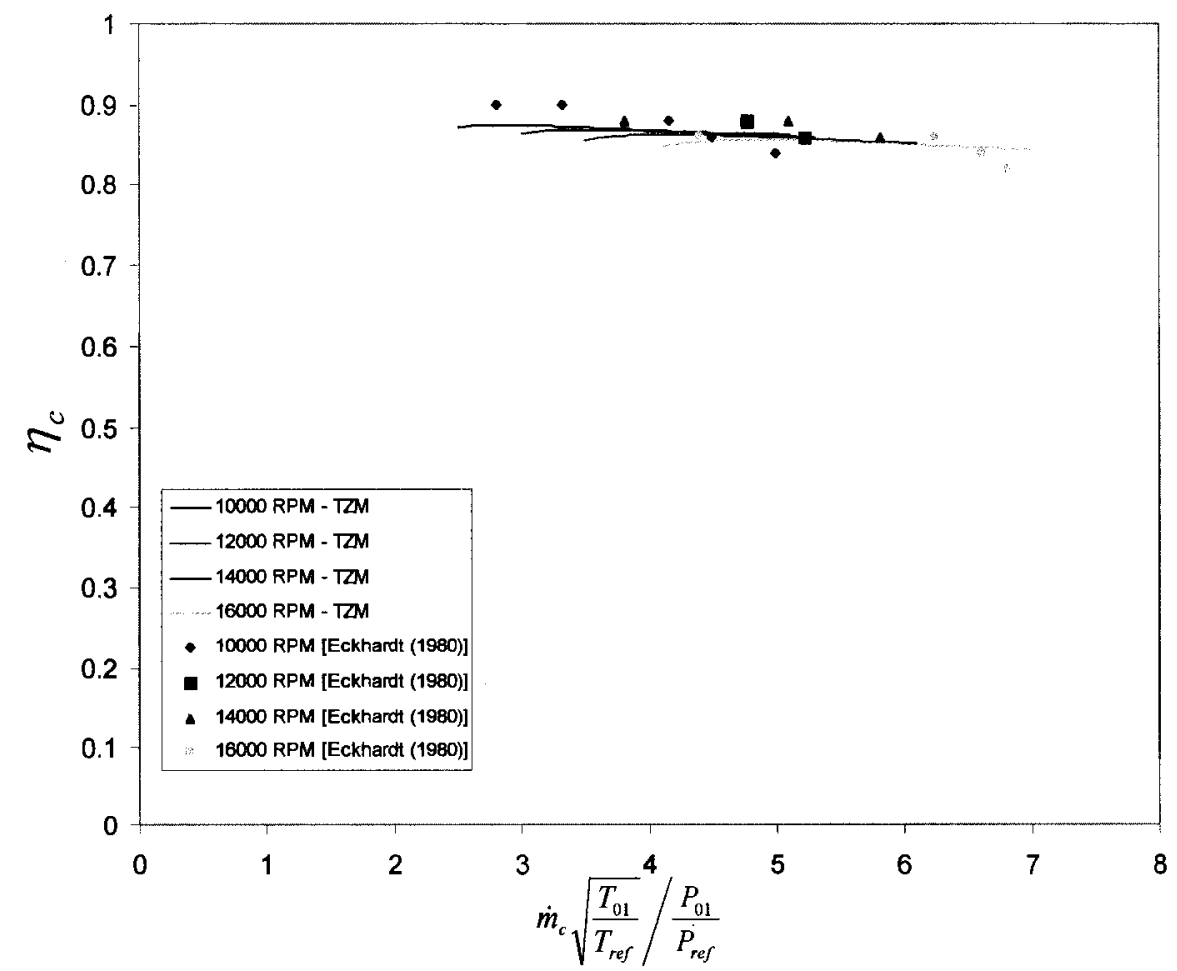

Figure 4.6 Isentropic efficiency of Eckhardt impeller A. 


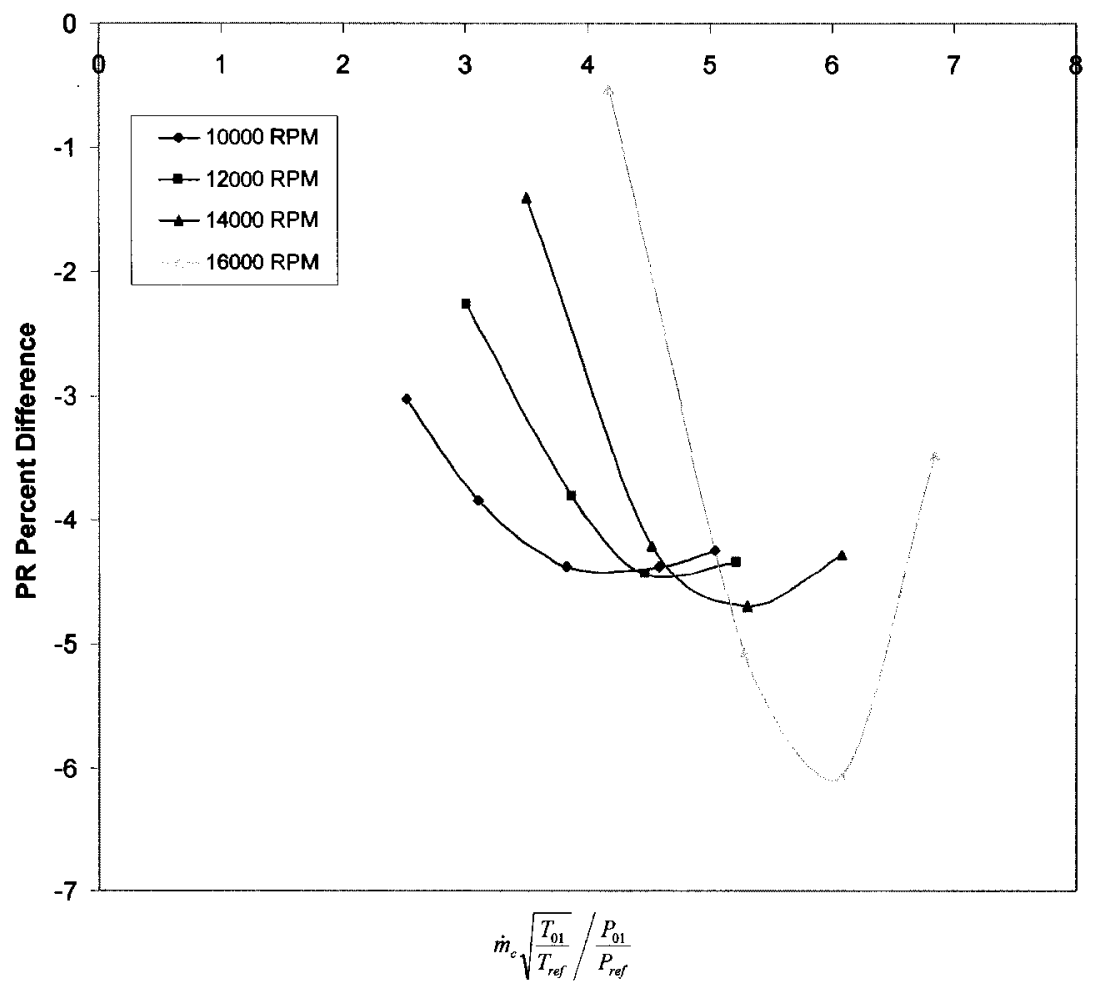

Figure 4.7 Difference between pressure ratio generated by TZM and the value from the actual impeller map for Eckhardt impeller A.

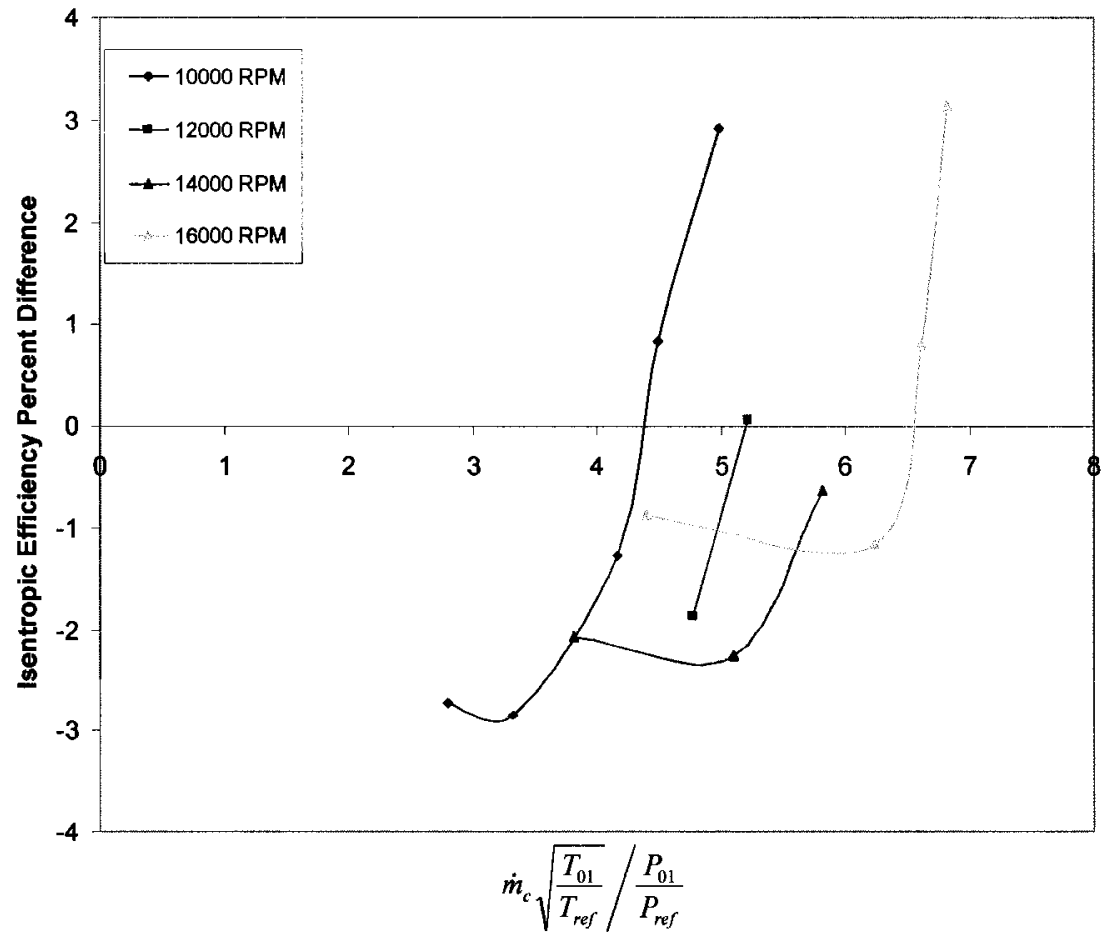

Figure 4.8 Difference between efficiency generated by TZM and the value from the actual impeller map for Eckhardt impeller A. 
Figures 4.5 through 4.8 show a similar level of accuracy for Eckhardt impeller A in comparison with the results of Eckhardt impeller O. Again, predication accuracy decreases with increasing rotational speed, however the pressure ratio, as determined by the two-zone model, is maintained within 6 percent of the actual map value. Isentropic efficiency is predicted well again, within 3 percent of the actual map values for all rotational speeds considered. Of particular contrast to Eckhardt impeller $O$ is the underprediction of pressure ratio for impeller $\mathrm{A}$ as opposed to the over-prediction of pressure ratio for impeller $\mathrm{O}$.

The following six figures show the performance of the impeller described by McKain and Holbrook as well as the results presented by Larosiliere et al. (1997). The impeller itself was scaled up from a previous design (DDA 404-III compressor), which operated at a design flow of $3.655 \mathrm{lbm} / \mathrm{s}(1.66 \mathrm{~kg} / \mathrm{s})$, to the required $10 \mathrm{lbm} / \mathrm{s}(4.54 \mathrm{~kg} / \mathrm{s})$. Figures 4.9 and 4.10 show the pressure ratio and efficiency characteristics for the design speed. Characteristics are shown for the actual map value, the map value as calculated by two-zone modeling (TZM) as well as the characteristic as determined by CFD techniques. Figures 4.11 and 4.12 indicate the difference between the actual map value and the value calculated by the two-zone model for both pressure ratio and isentropic efficiency. 


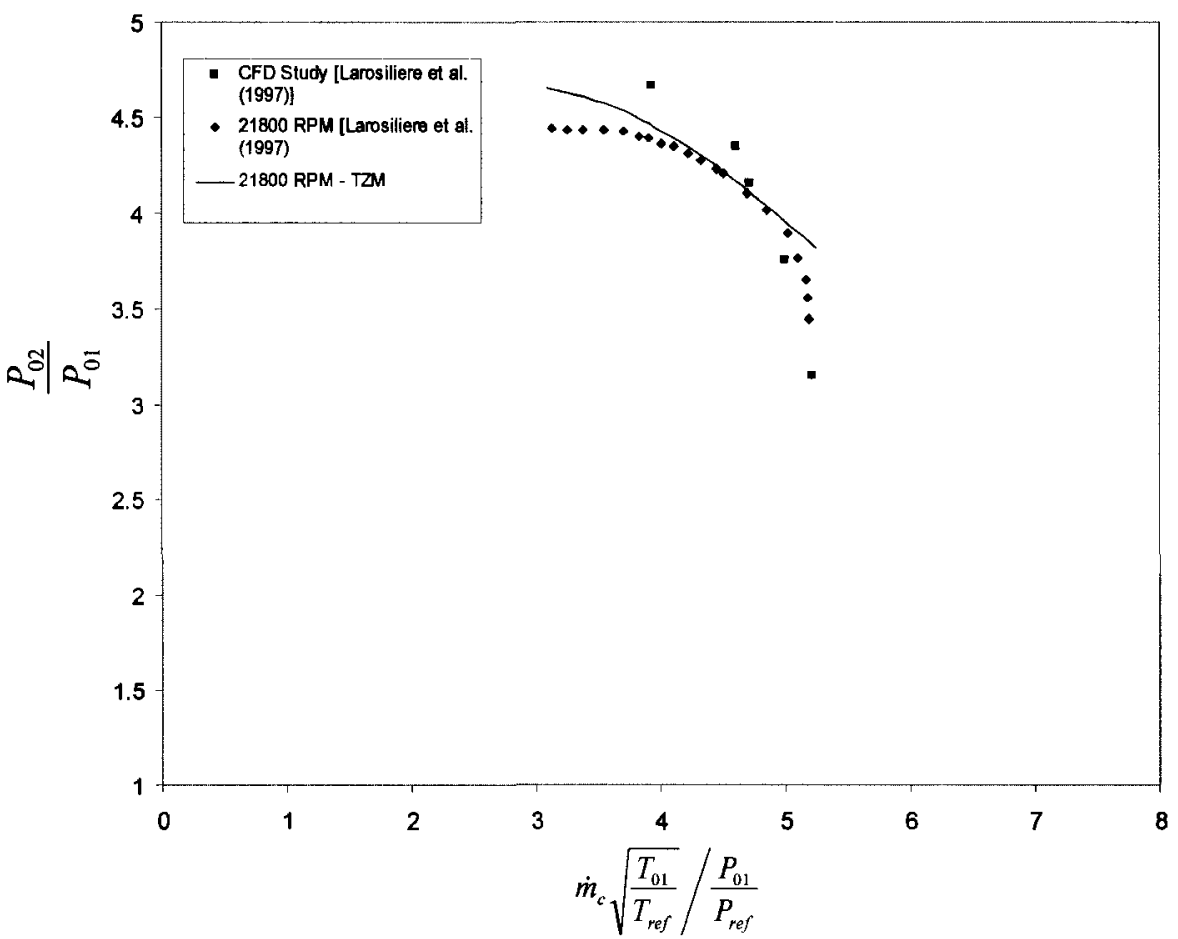

Figure 4.9 Pressure ratio of the impeller described by McKain and Holbrook (1997).

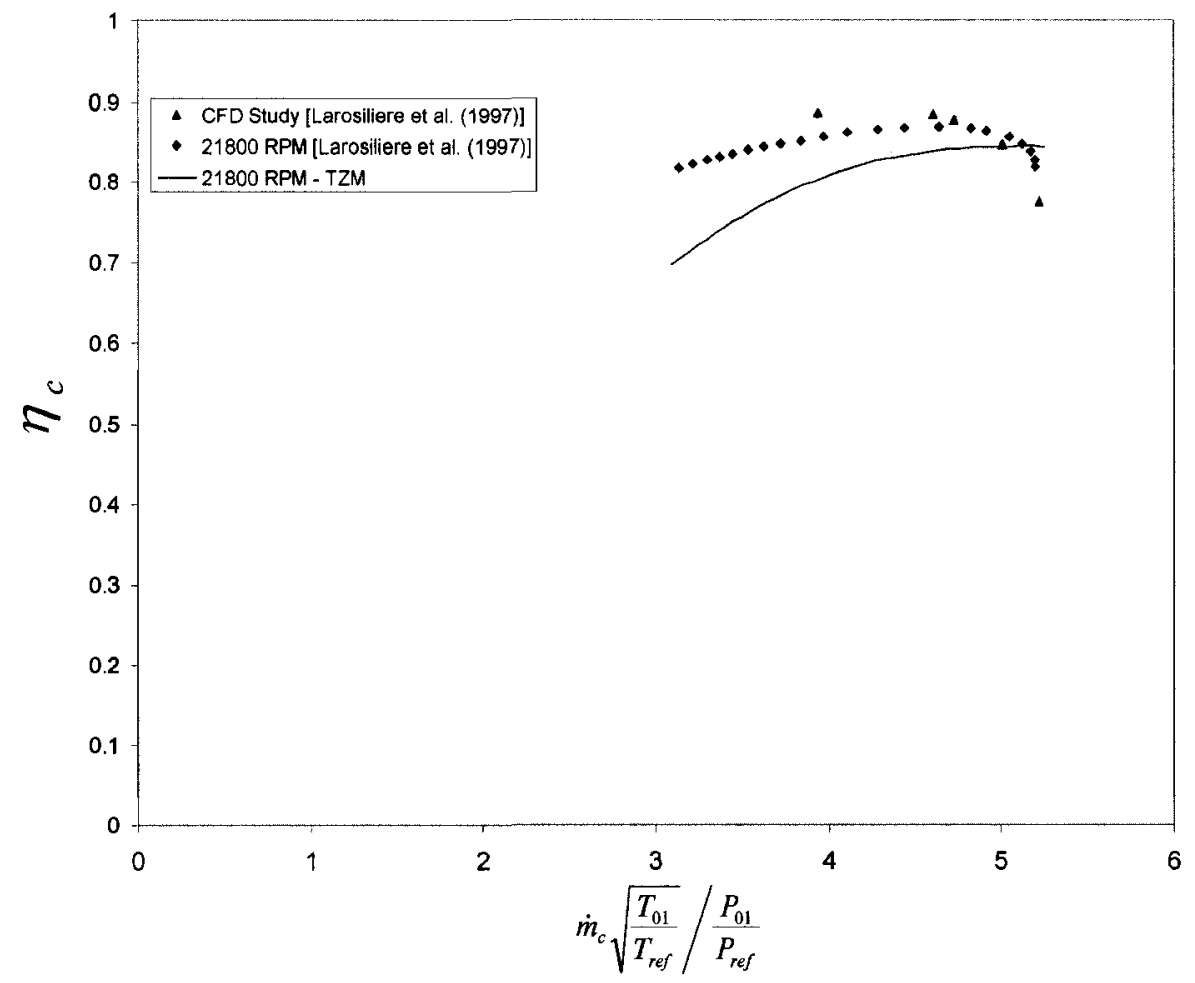

Figure 4.10 Isentropic efficiency of the impeller described by McKain and Holbrook (1997). 


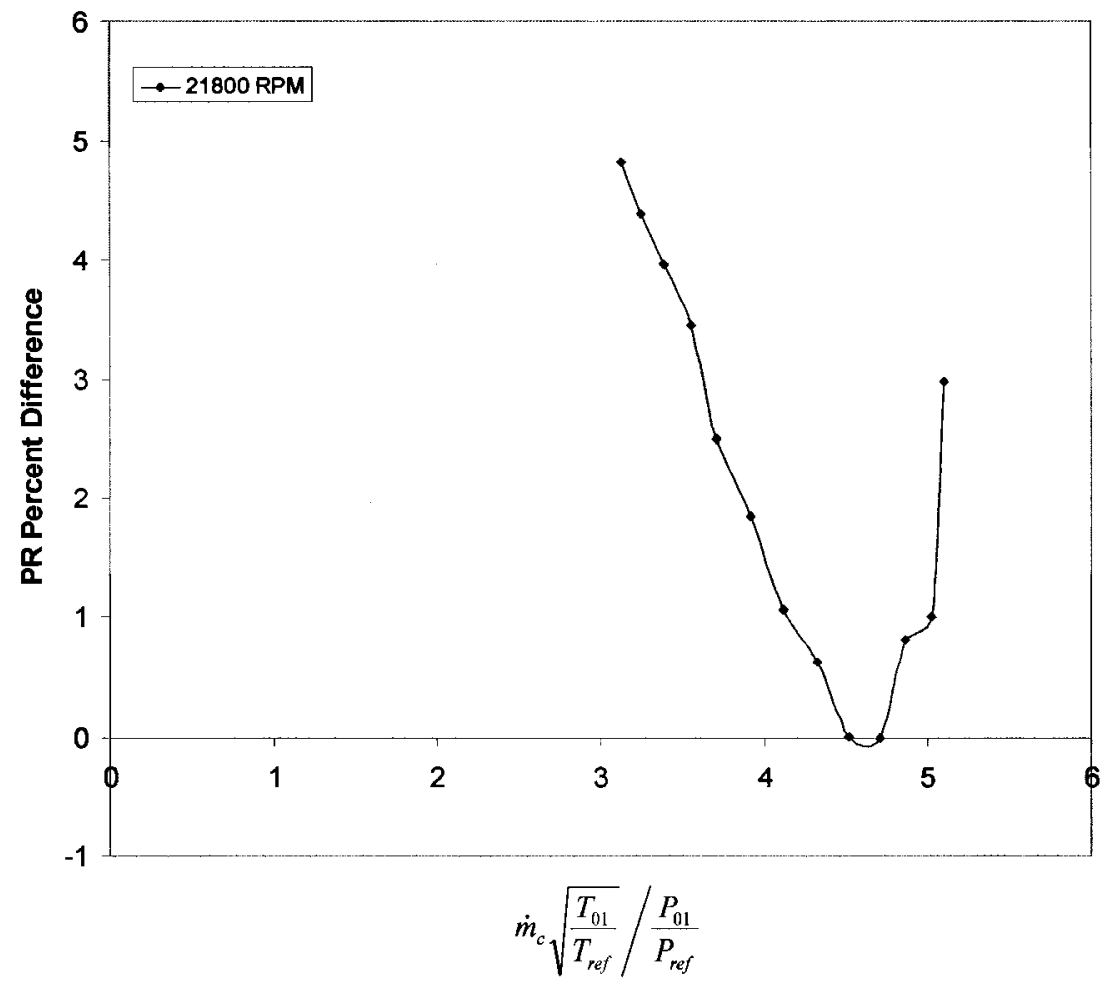

Figure 4.11 Difference between the pressure ratio generated by TZM and the value from the actual impeller map for the impeller described by McKain and Holbrook (1997).

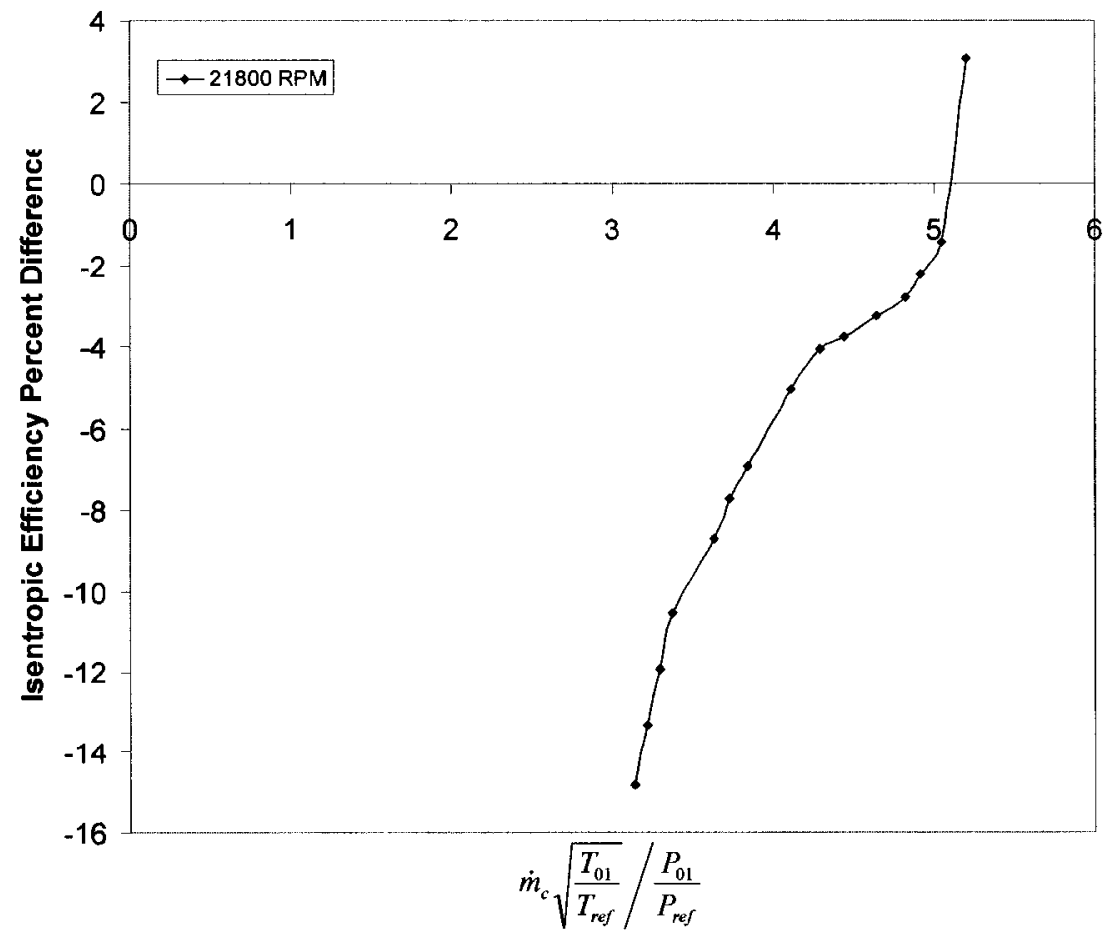

Figure 4.12 Difference between the efficiency generated by TZM and the value from the actual impeller map for the impeller described by McKain and Holbrook (1997). 
Figures 4.9 through 4.12 show the performance of the intermediate pressure ratio impeller described by McKain and Holbrook (1997). Unfortunately, only the design speed line was available for comparison for this particular impeller, however results indicate good agreement between the actual design speed characteristic and the design speed characteristic generated by the two-zone model. Figure 4.11 shows a maximum difference of 5 percent between the actual pressure ratio and the pressure ratio generated by the two-zone model. Of particular interest in Figures 4.9 and 4.10 is the comparison of the characteristic generated by the TZM and the characteristic generated by a CFD analysis of the impeller. Specifically Figure 4.9 shows that the two-zone model predicts the impeller behaviour as well as, or better than, the CFD analysis performed by Larosiliere et al. (1997) to determine the characteristic. However more comparisons need to be made before a general conclusion can be drawn regarding the accuracy of the twozone model in comparison with CFD techniques.

As with both Eckhardt impellers, a full map could be obtained for the impeller described by McKain and Holbrook (1997). Figures 4.13 and 4.14 show the ability of the two-zone model to predict values outside what is presented; pressure ratio is predicted for corrected mass flow rates between 2 and $7 \mathrm{~kg} / \mathrm{s}$ and for rotational speeds between 62 percent and 103 percent of the design speed. 


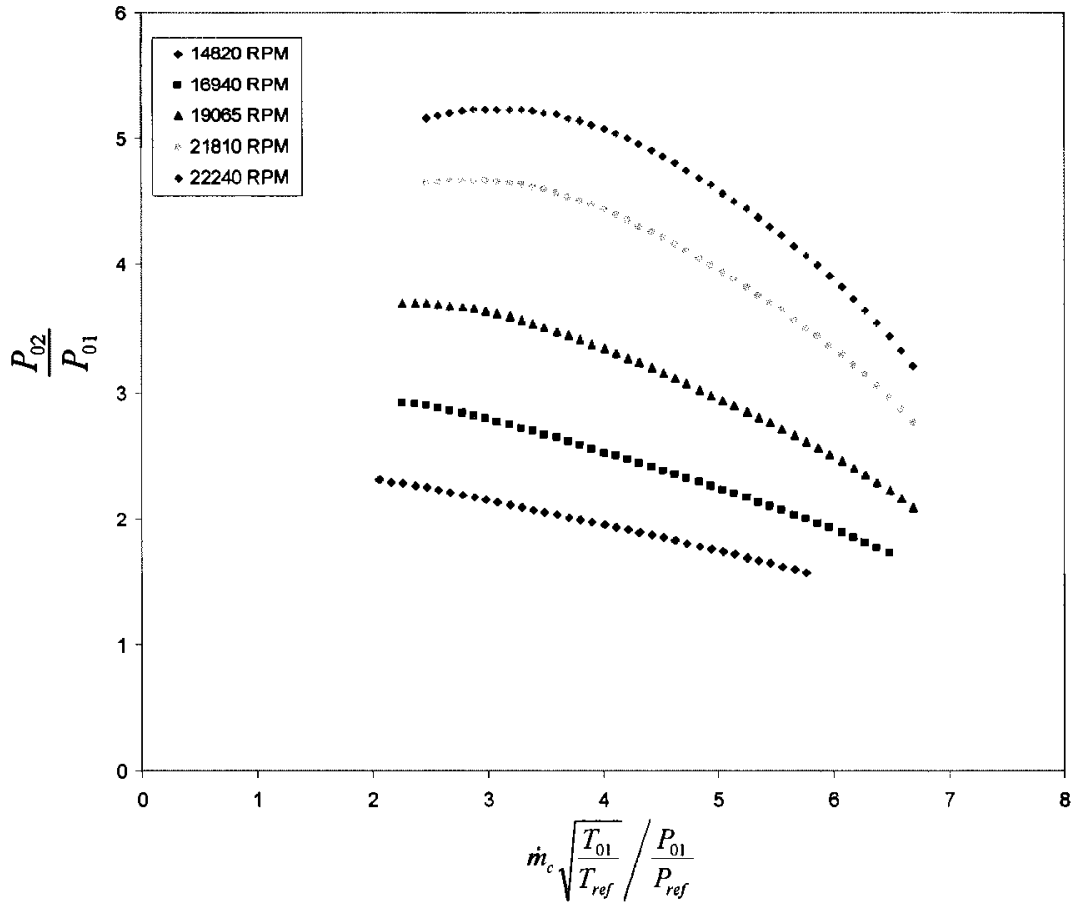

Figure 4.13 Pressure ratio characteristics describing the rotational speed limits of the two-zone model for the impeller described by McKain and Holbrook (1997).

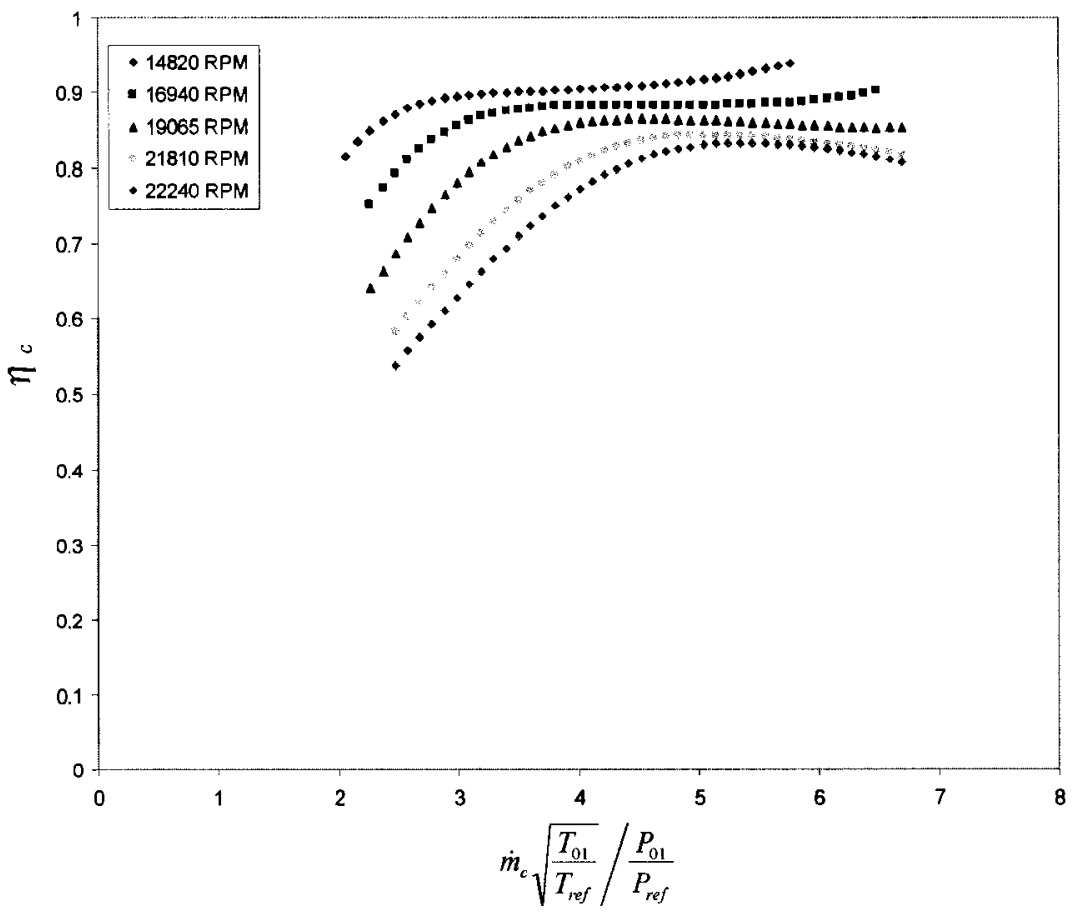

Figure 4.14 Isentropic efficiency characteristics describing the rotational speed limits of the two-zone model for the impeller described by McKain and Holbrook (1997). 
The following four figures show the performance of the last impeller tested; a high pressure ratio impeller which was described and presented by Came and Herbert (1980). The impeller was adapted from a previous impeller design which originally operated at a design point pressure ratio of $6.5: 1$, subsequently modified to a design pressure ratio of $8: 1$. Figures 4.15 and 4.16 show the pressure ratio and efficiency characteristics, respectively; actual map characteristics were compared against those generated by the two-zone model. Figures 4.17 and 4.18 indicate the difference of pressure ratio and efficiency between the actual map value and the map value as calculated by the two-zone model.

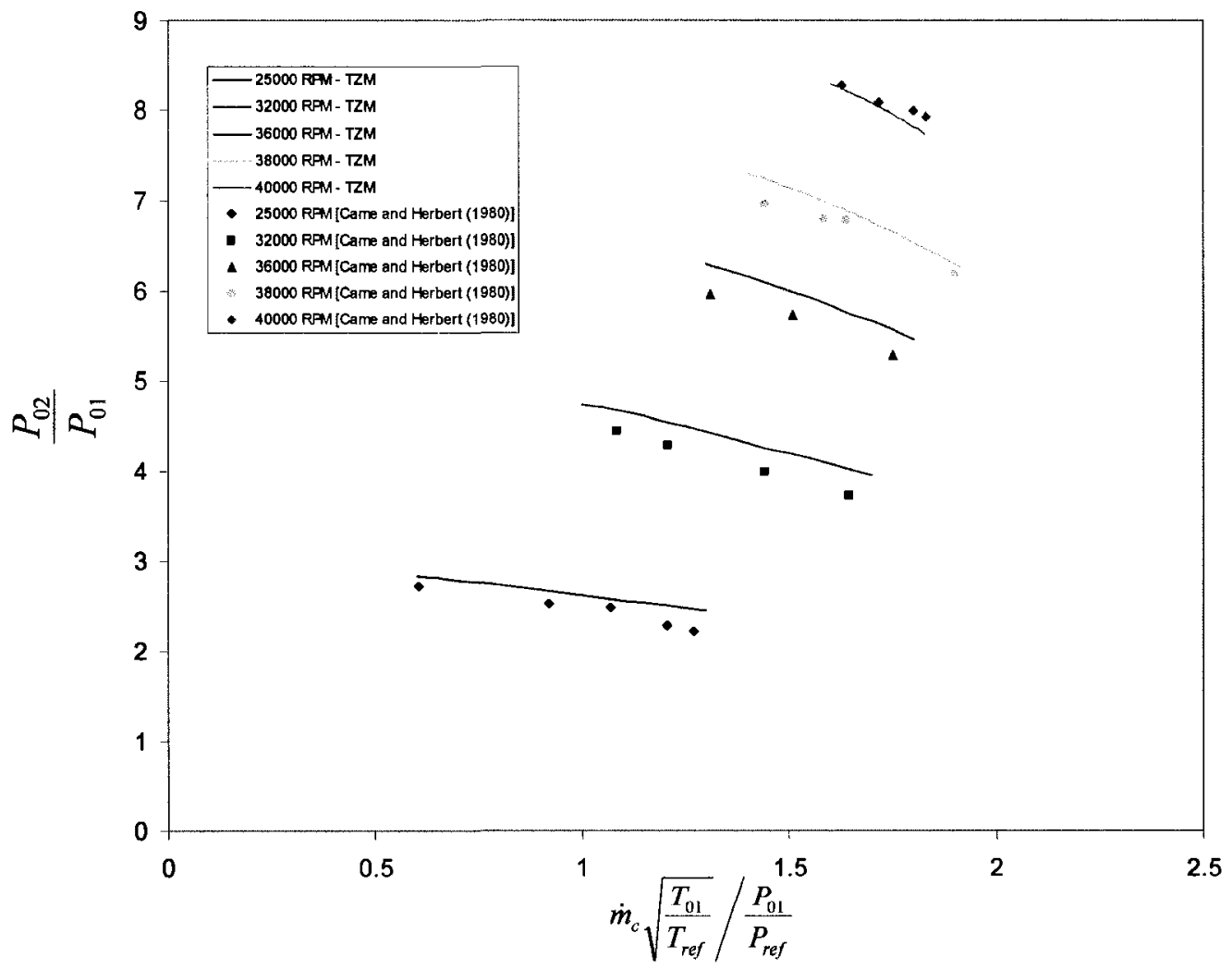

Figure 4.15 Pressure ratio of the impeller described by Came and Herbert (1980). 


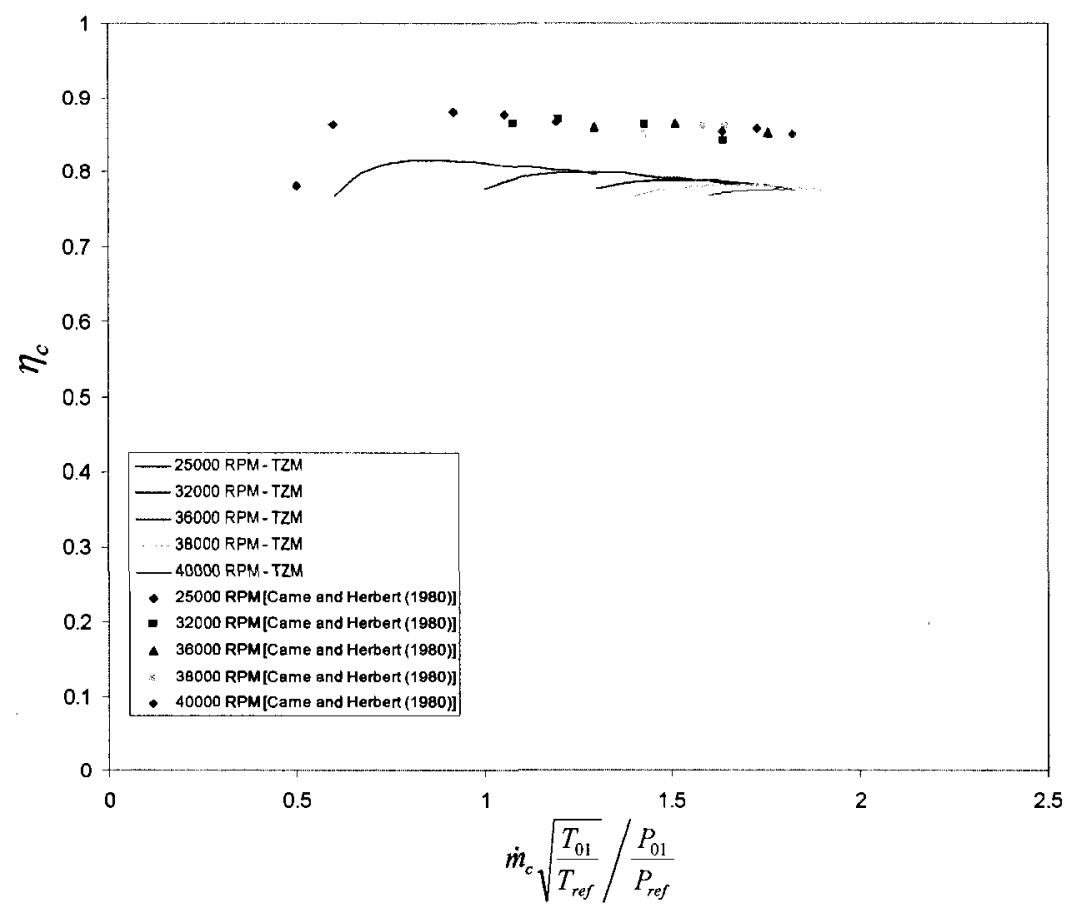

Figure 4.16 Isentropic efficiency of the impeller described by Came and Herbert (1980).

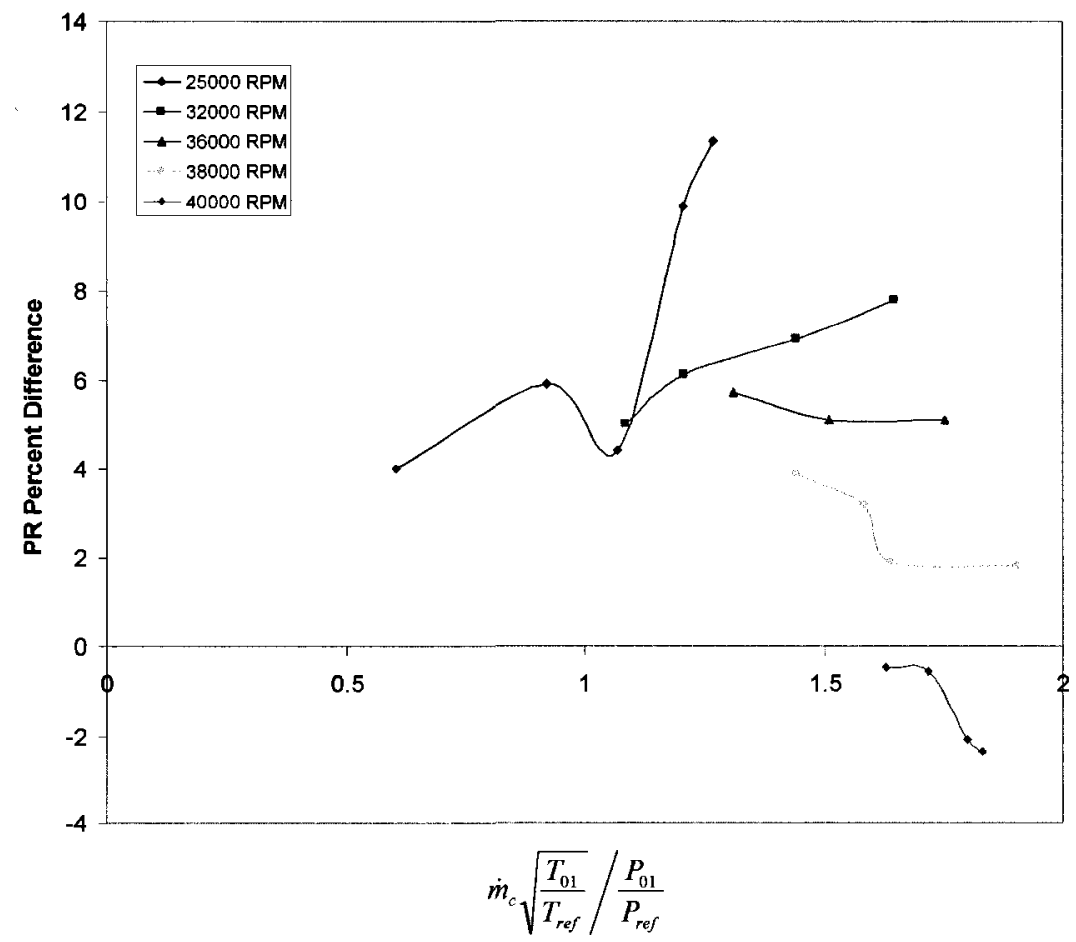

Figure 4.17 Difference between pressure ratio generated by TZM and the value from the actual impeller map described by Came and Herbert (1980). 


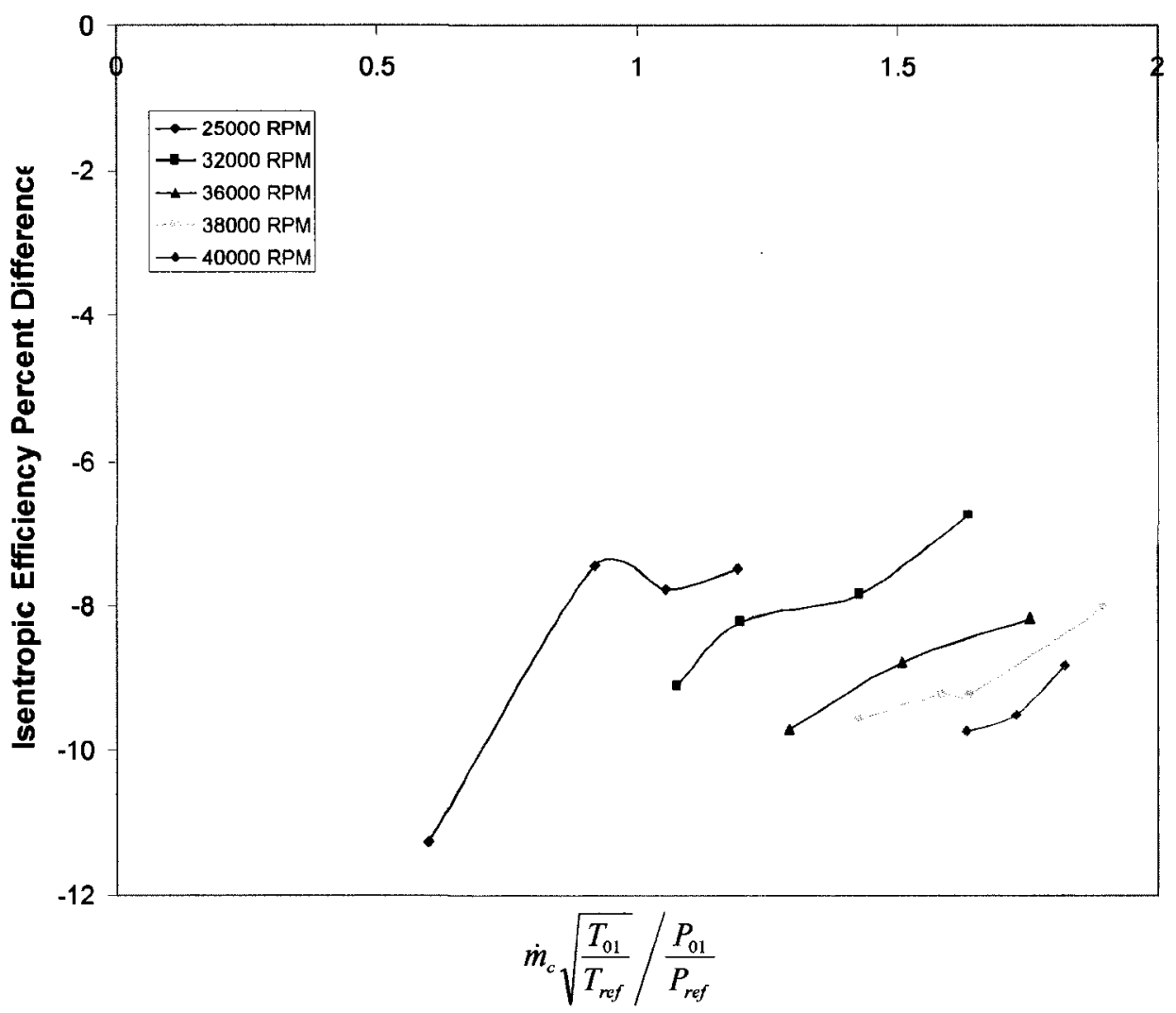

Figure 4.18 Difference between isentropic efficiency generated by TZM and the value from the actual impeller map described by Came and Herbert (1980).

Figure 4.15 through 4.18 show the final impeller tested using the two-zone model, a high pressure ratio impeller described by Came and Herbert (1980). Figures 4.15 and 4.17 show good agreement between the two-zone model and the actual impeller map at high rotational speeds, however the difference approaches 12 percent on the lowest speed characteristic. Isentropic efficiency is predicted less well; the difference between the actual efficiency characteristics and the efficiency characteristics generated by the twozone model lie between 6 percent and 12 percent over the whole range of operating conditions. 
The two-zone model was able to be pushed beyond what is presented in Figures 4.15 through 4.18. Speed characteristics were able to be obtained as low as 50 percent of the design speed and as high as 112 percent of the design speed, however the analysis was highly sensitive to the input corrected mass flow.

The accuracy of the two-zone model in determining the performance of impellers at off-design conditions is very dependant upon three parameters: 1) effectiveness of the impeller inlet, $\eta_{a}$ (section 3.1.2), 2) effectiveness of the impeller passage, $\eta_{b}$ (section 3.1.2) and 3) the wake mass flow fraction $(\chi)$. Both effectiveness parameters at the moment are user provided, therefore, selection of the parameters is highly dependant upon user experience with the model. For instance, values of 1.05 and 0.30 for $\eta_{a}$ and $\eta_{b}$ show good agreement with the actual map values of Came and Herbert (1980) at higher rotational speeds, however values of 1.0 and 0.4 for $\eta_{a}$ and $\eta_{b}$ show good agreement with actual map values of Came and Herbert (1980) at lower rotational speeds.

Recently, an investigation was performed by R.J. Pelton (2007) into the possible prediction of the effectiveness values for use in the TEIS model in conjunction with twozone modelling. A linear regression model was performed and the statistical relevance of each variable in the model was examined to ascertain its applicability in the model. Ultimately, it was determined by Pelton that the inlet effectiveness is influenced by:

- Inlet tip Reynolds number

- Ratio of inlet blade height to inlet tip Reynolds number

- Ratio of passage length-to-hydraulic diameter 
- Impeller exit relative Rossby number

- Exit blade clearance

- Inlet tip incidence

- Inlet tip flow angle

- Number of blades

- Impeller inlet meridional velocity ratio (tip-to-RMS value)

- Inlet relative rotational number

According to Pelton (2007), passage effectiveness is influenced by:

- Ratio of passage length-to-hydraulic diameter

- Ratio of exit area-to-inlet area

- Machine type (compressor or pump)

- Ratio of exit blade height-to-outlet radius

- Ideal outlet absolute Rossby number

- Exit blade clearance

\subsection{Low Speed Extrapolation Validation}

Testing of the low speed extrapolation algorithm was performed utilizing the existing maps used for testing of the impeller two-zone model. Eckhardt $O$ and Eckhardt A impeller, as well as the impeller described by Came and Herbert (1980) were used specifically. As outlined in Chapter 3, the low speed extrapolation algorithm uses two reference speed lines to calculate the extrapolation exponents $\mathrm{p}, \mathrm{q}$ and $\mathrm{r}$ for each beta line. 
Validation of the algorithm was performed by using two of the higher speed lines in a compressor map to predict the lower speed lines. Figures 4.19 through 4.24 show the comparison of the actual map values obtained from the literature, the pressure ratio characteristics and efficiency characteristics as predicted by the two-zone model and the speed lines predicted by the low speed extrapolation method.

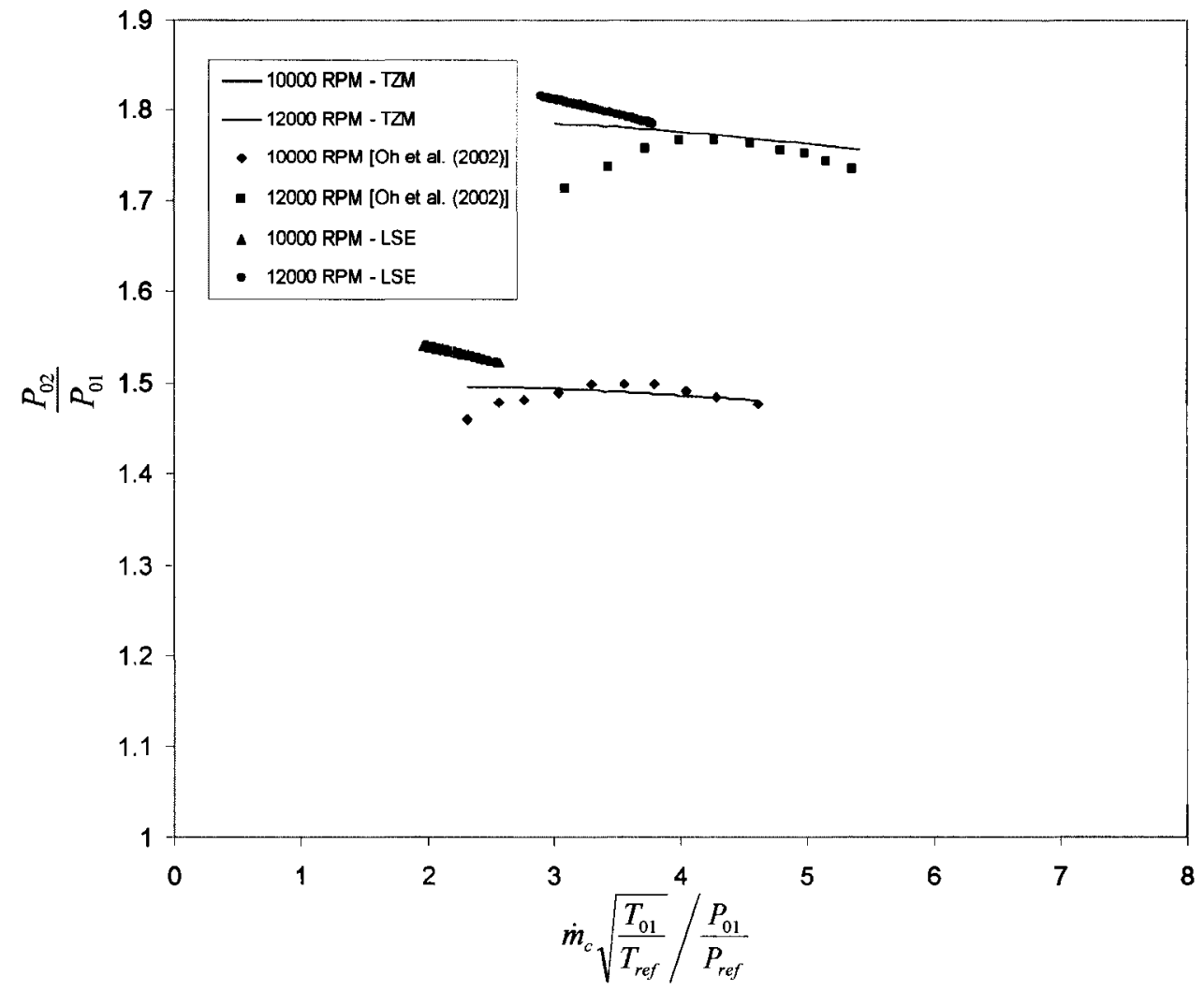

Figure 4.19 Actual map, TZM and LSE pressure ratio characteristics for Eckhardt impeller O. 


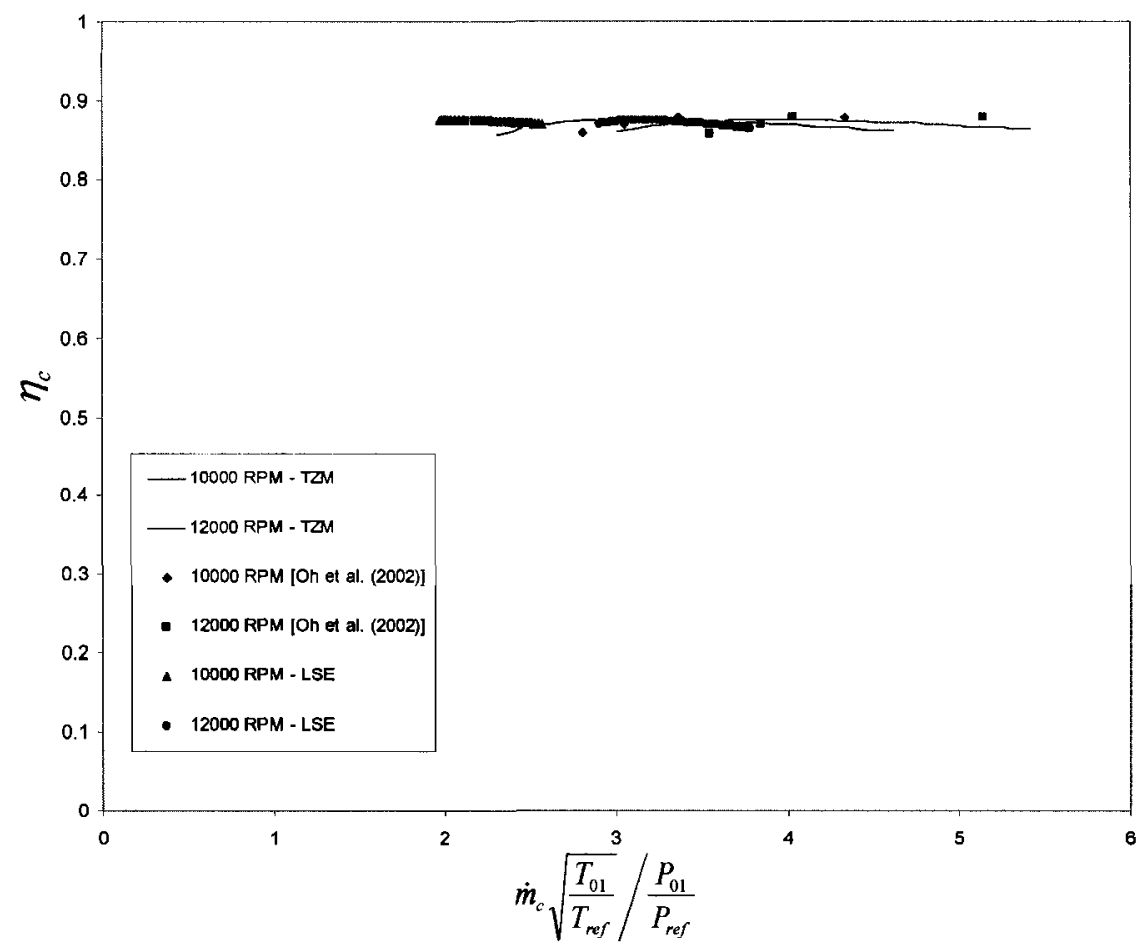

Figure 4.20 Actual map, TZM and LSE efficiency characteristics for Eckhardt impeller O.

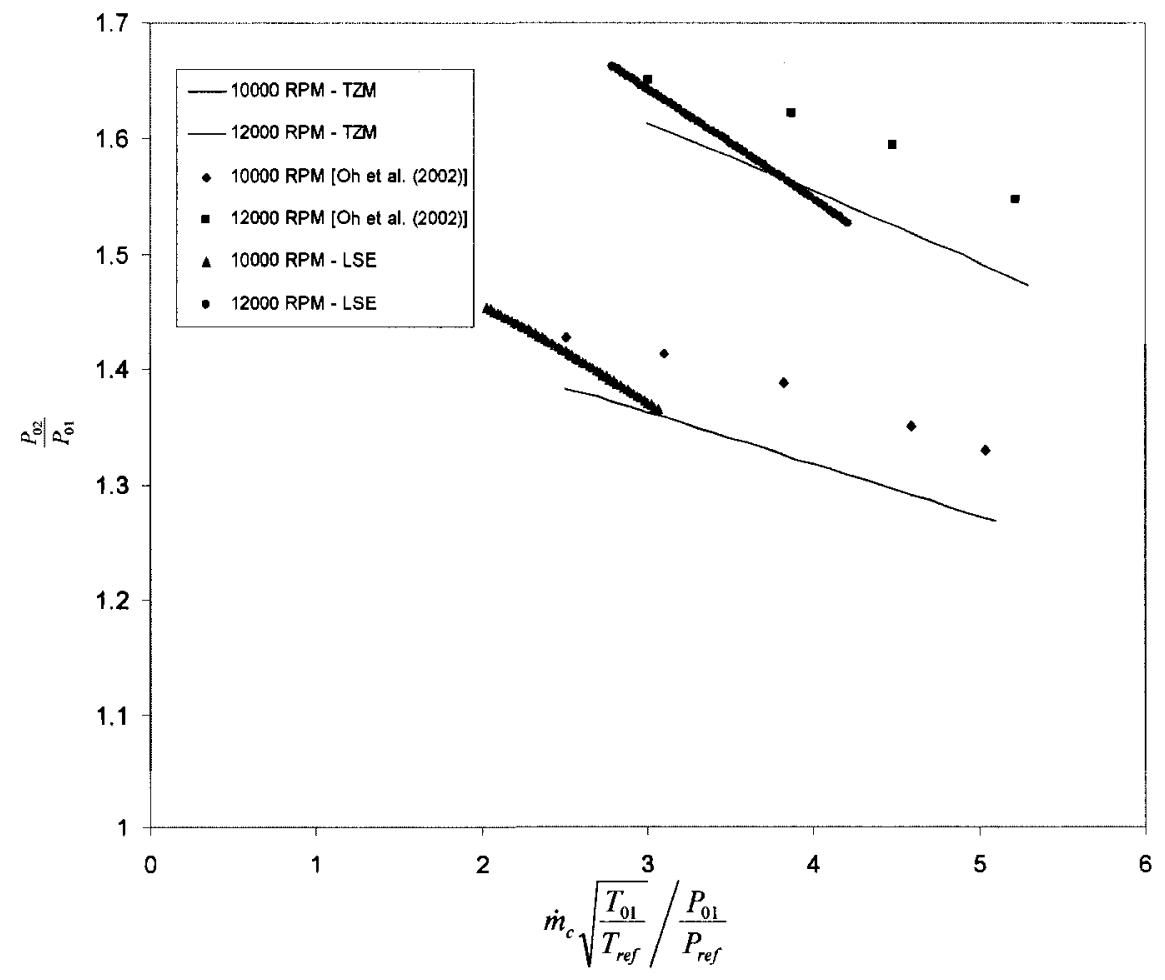

Figure 4.21 Actual map, TZM and LSE pressure ratio characteristics for Eckhardt impeller A. 


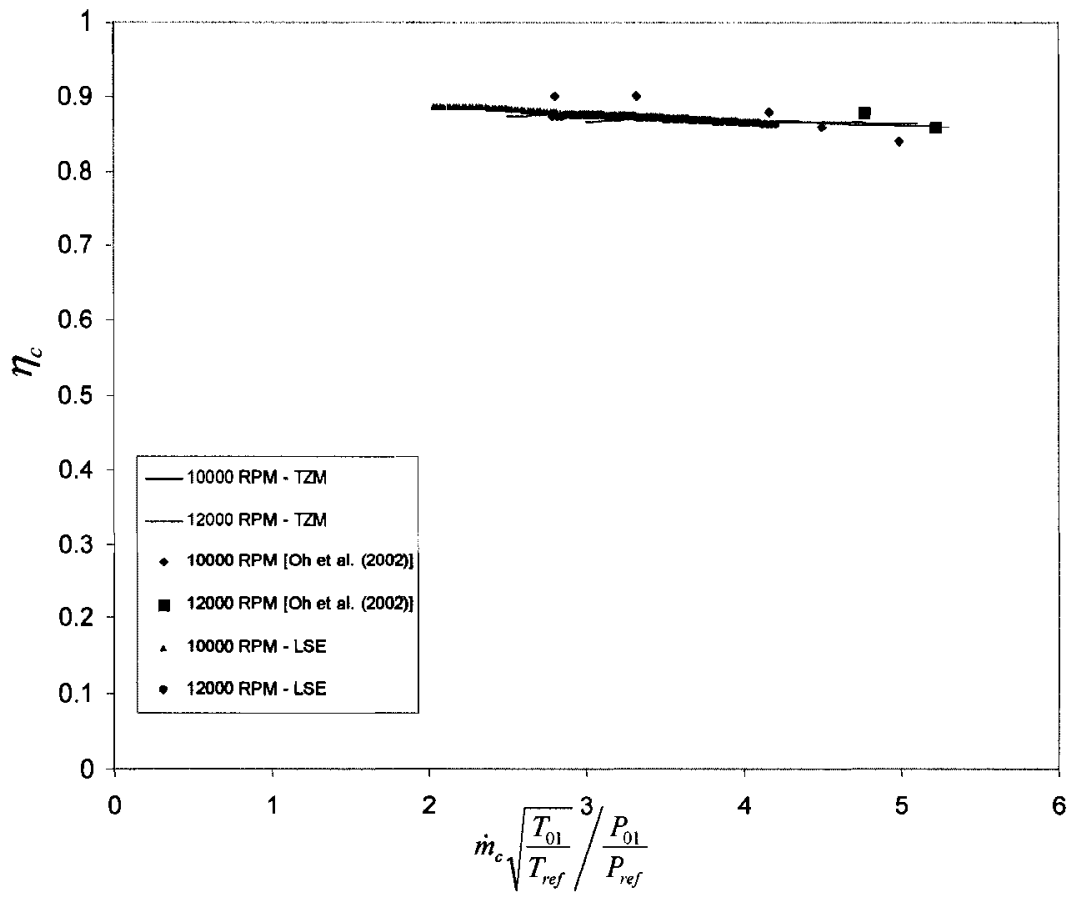

Figure 4.22 Actual map, TZM and LSE efficiency characteristics for Eckhardt impeller A.

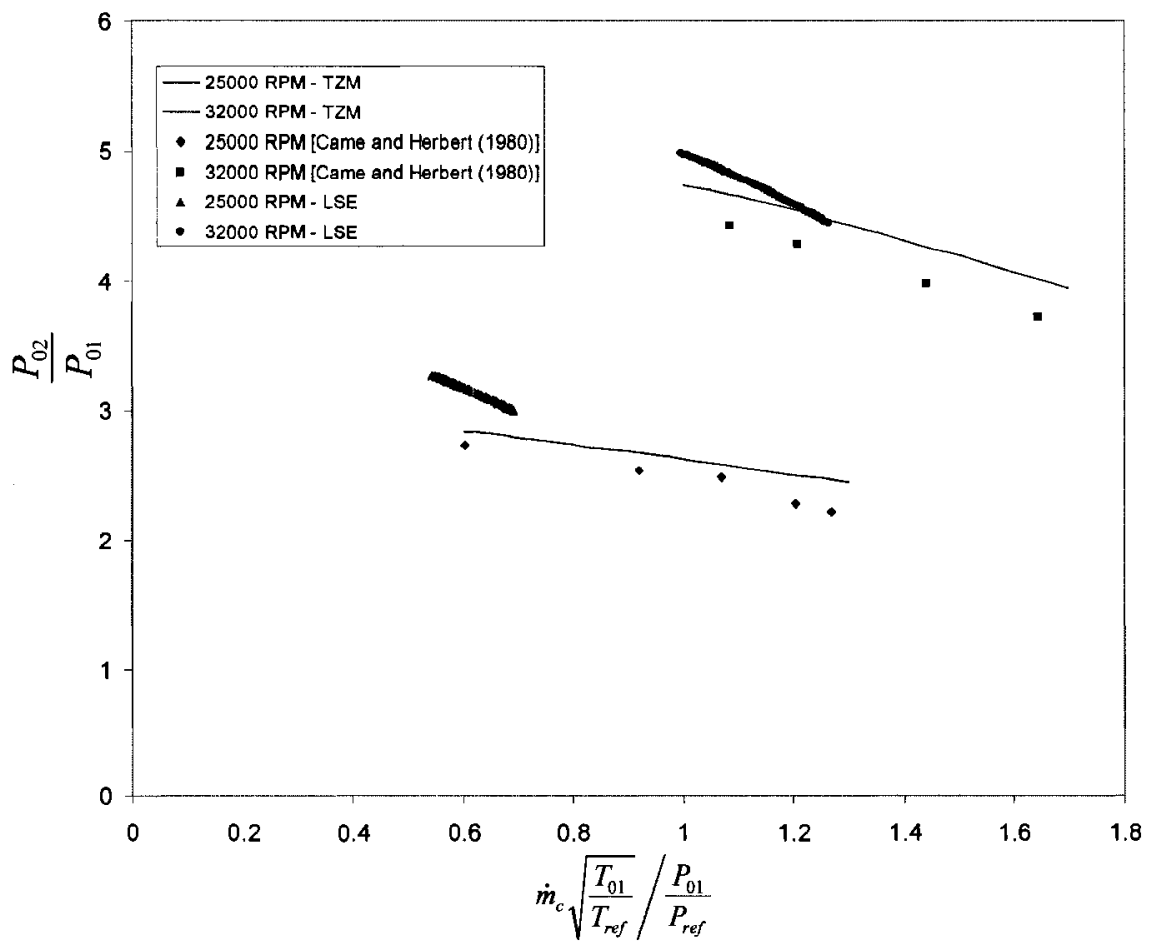

Figure 4.23 Actual map, TZM and LSE pressure ratio characteristics for the impeller described by Came and Herbert (1980). 


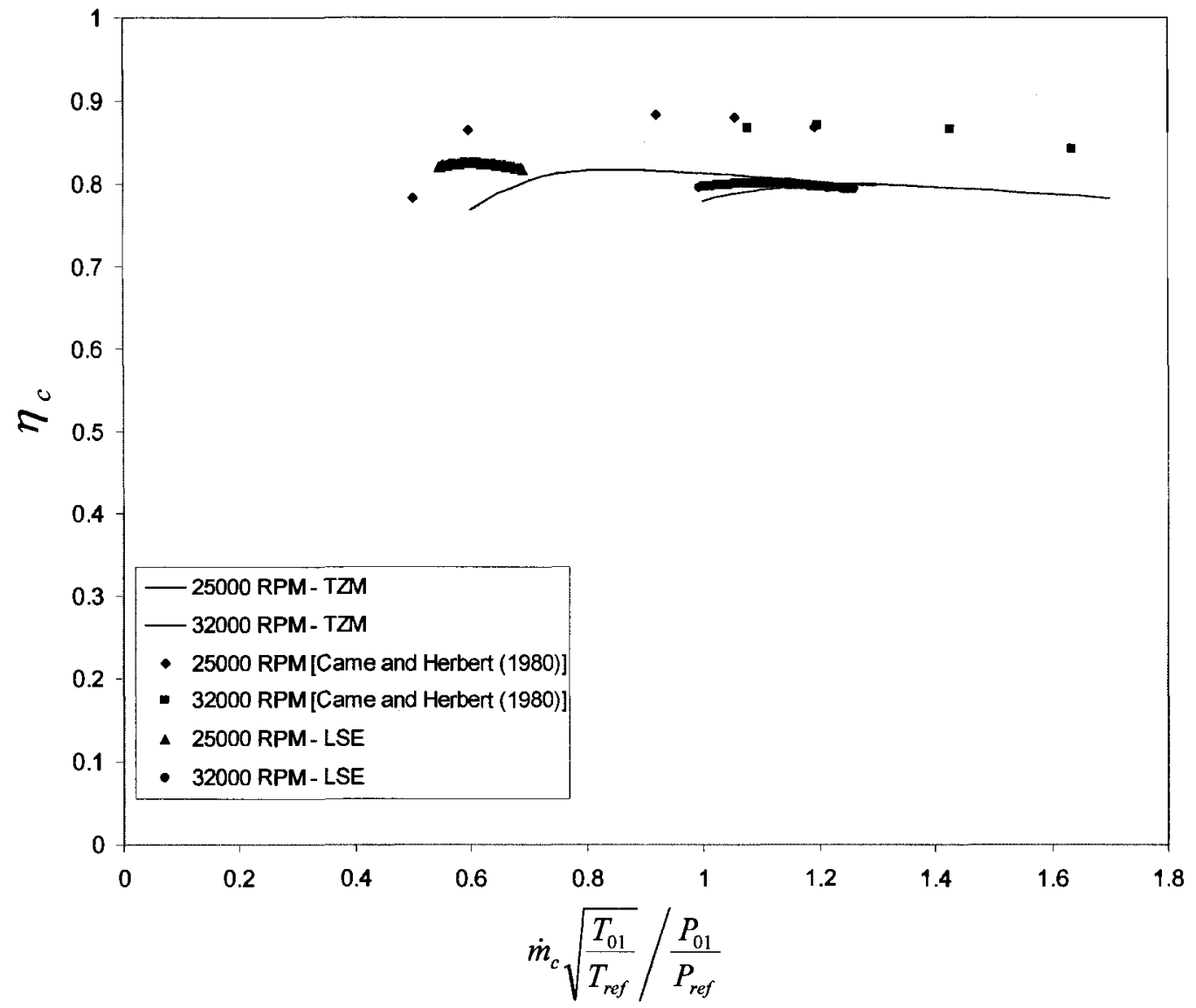

Figure 4.24 Actual map, TZM and LSE efficiency characteristics for the impeller described by Came and Herbert (1980).

Of all three impellers tested, all show similar trends and characteristics. The correct magnitudes for pressure ratio and isentropic efficiency are determined by the low speed extrapolation algorithm, however the range over which the characteristics run is not well approximated. All impellers show a very narrow range for the characteristics determined by the low speed extrapolation algorithm beyond what is expected from the actual map. Despite the poor results on the range of the mass flow rates, the low speed algorithm still shows promise in that it is able to predict the relative magnitudes correctly. It is hypothesized that improvement of the beta grid algorithm with the use of parabolas as the beta grid, as opposed to the straight lines employed here, could improve the results 
generated by the low speed extrapolation method. This assumption is only preliminary however, and further investigation is required to refine the technique further.

Furthermore, the low speed extrapolation technique is not essential to the overall model and the performance results generated; the low speed extrapolation algorithm was implemented for compressor map completion to the low speed region. At present, the author is comfortable with its present use along with the remainder of the models for use with the overall engine performance estimation tool.

\subsection{Performance Prediction Validation}

The third aspect of the validation process required the estimation of the accuracy of the performance prediction algorithm when compared against more accepted methods used in gas turbine performance prediction. Scaled compressor maps were obtained from $\operatorname{GasTurb}^{\mathrm{TM}}$ and were subsequently curve-fit and imported into the performance prediction algorithm coded in-house. Results from the calculation of the equilibrium running line from the in-house code were compared against those results from the calculation of the equilibrium running line in GasTurb ${ }^{\mathrm{TM}}$ to generate confidence in the in-house model.

A ground-based, single shaft, gas turbine engine was selected from GasTurb ${ }^{\mathrm{TM}}$ for comparison. All pertinent information used in the GasTurb ${ }^{\mathrm{TM}}$ model is shown in Table 4.2 . 
Table 4.2 Data used in GasTurb ${ }^{\mathrm{TM}}$ single shaft engine simulation.

\begin{tabular}{|l|l|}
\hline \multicolumn{1}{|c|}{ Quantity Name } & \multicolumn{1}{c|}{ Value } \\
\hline Total Inlet Temperature & $288.15 \mathrm{~K}$ \\
\hline Total Inlet Pressure & $101.325 \mathrm{kPa}$ \\
\hline Ambient Pressure & $101.325 \mathrm{kPa}$ \\
\hline Relative Humidity & 0 \\
\hline Inlet Corrected Mass Flow & $5 \mathrm{~kg} / \mathrm{s}$ \\
\hline Intake Pressure Ratio & 0.99 \\
\hline Compressor Pressure Ratio & 4.5 \\
\hline Burner Exit Temperature & $1400 \mathrm{~K}$ \\
\hline Burner Efficiency & 0.99 \\
\hline Fuel Heating Value & $55.528 \mathrm{MJ} / \mathrm{kg}$ \\
\hline Overboard Bleed & 0 \\
\hline Mechanical Efficiency & 0.9999 \\
\hline Burner Pressure Ratio & 0.97 \\
\hline Turbine Exit Duct Pressure Ratio & 1 \\
\hline Design Exhaust Pressure Ratio & 1 \\
\hline Isentropic Compressor Efficiency & 0.8181 \\
\hline Nominal Spool Speed & $20000 \mathrm{RPM}$ \\
\hline Isentropic Turbine Efficiency & 0.8985 \\
\hline
\end{tabular}

The compressor map used in both performance prediction methods was that obtained from the GasTurb $^{\mathrm{TM}}$ software package and can be seen in Figure 4.25.

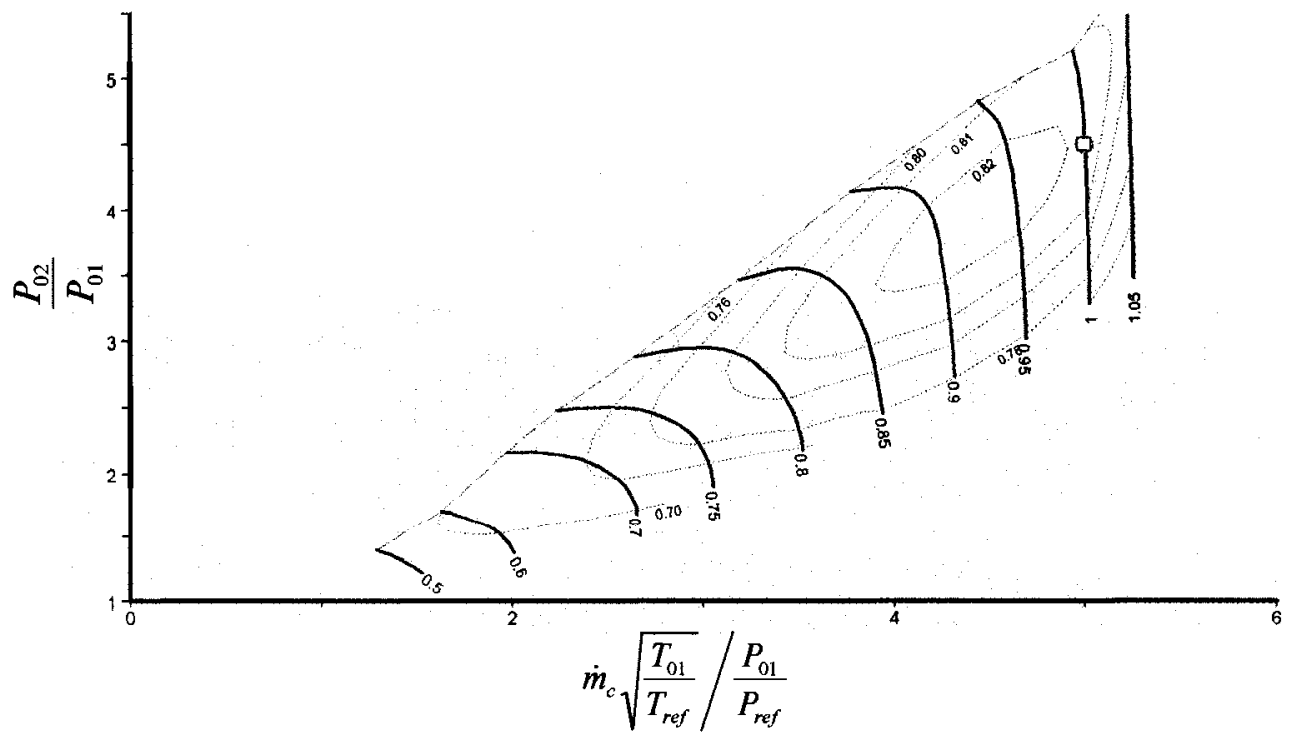

Figure 4.25 Compressor map obtained from GasTurb for use in the performance prediction algorithm. 
The running line was established both by the GasTurb ${ }^{\mathrm{TM}}$ code and by the performance prediction method coded in-house, a comparison between the two running lines can be seen in Figure 4.26.

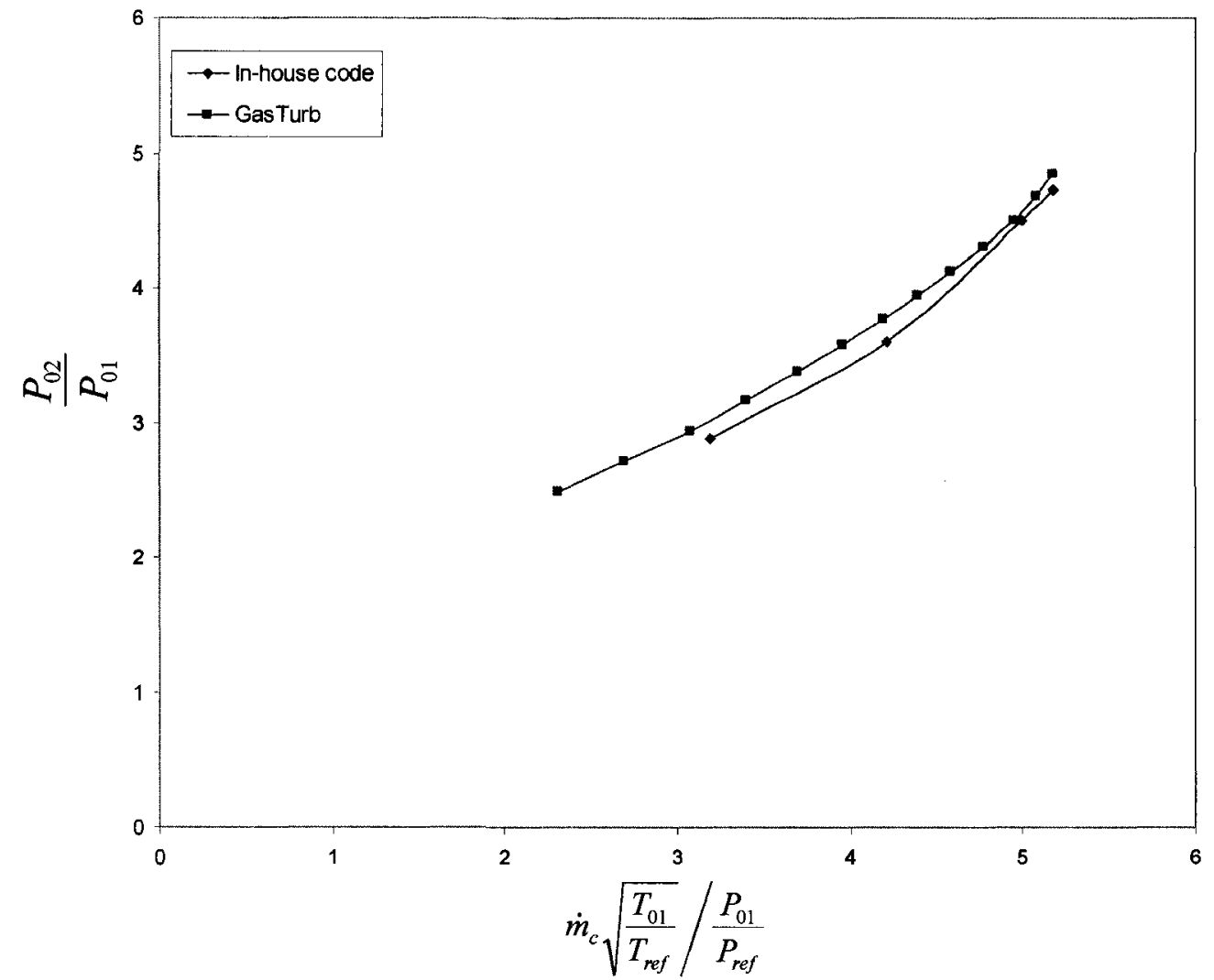

Figure 4.26 Equilibrium running lines determined by the GasTurb software package and the in-house code.

Figure 4.26 shows that the two running lines closely resemble one another, with the running line as determined by the in-house code passing through the exact design point, while the running line as determined by GasTurb ${ }^{\mathrm{TM}}$ is slightly offset. Due to the similarity, it is hypothesized that the performance prediction of the in-house code should resemble the performance prediction of the GasTurb ${ }^{\mathrm{TM}}$ package closely. Differences in the running line are attributed to the difference between the turbine map used by the 
GasTurb $^{\mathrm{TM}}$ software package and the turbine map used by the in-house performance model. Furthermore, it is not specified in the support files how exactly the running line is calculated by GasTurb ${ }^{\mathrm{TM}}$, therefore, it is possible there may be slight differences in the calculation process which would lead to a discrepancy between the running line as calculated by the in-house code and that as calculated by GasTurb ${ }^{\mathrm{TM}}$. Figures 4.27 through 4.29 show the engine performance as determined by the in-house code versus the engine performance as determined by GasTurb ${ }^{\mathrm{TM}}$.

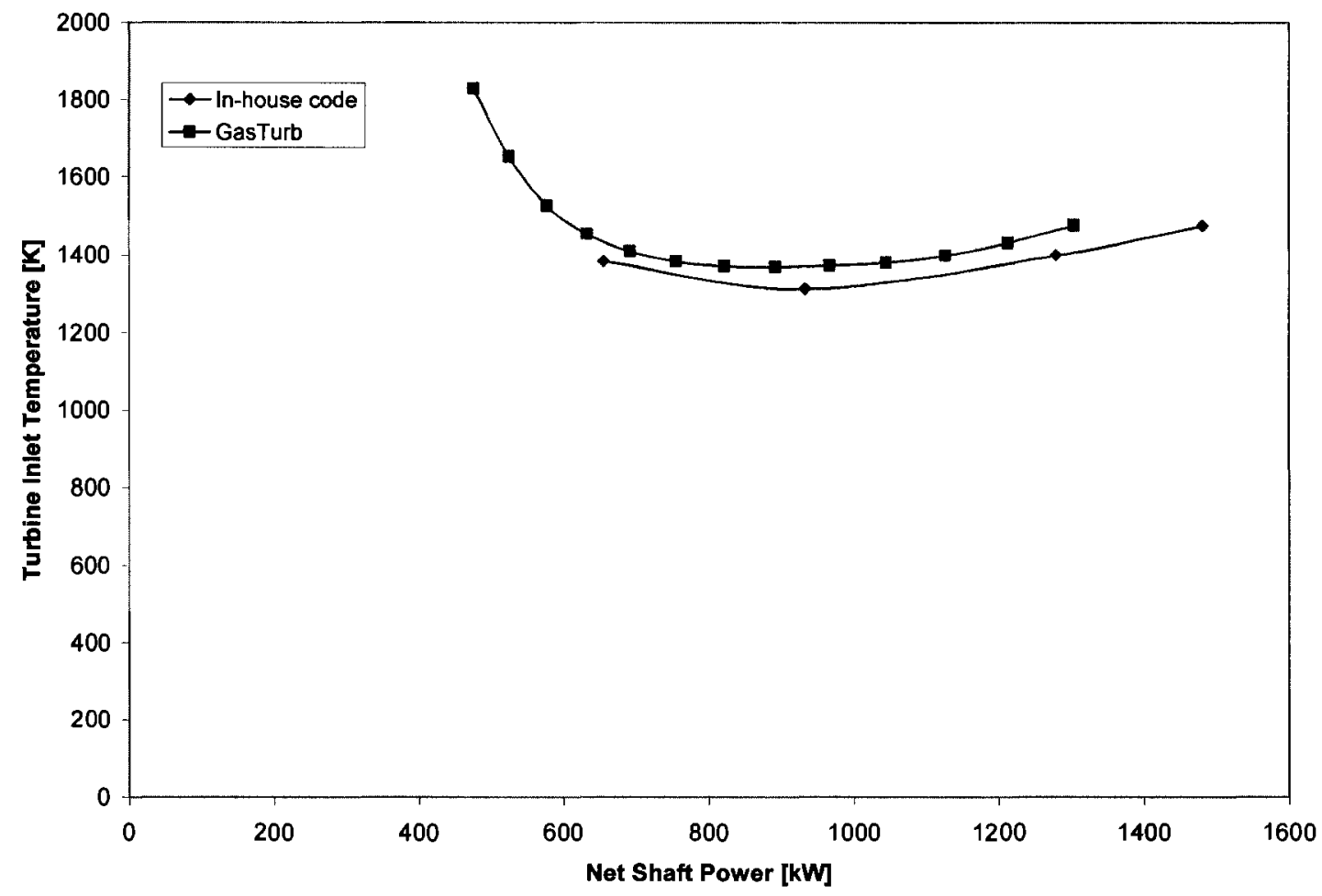

Figure 4.27 Variation of turbine inlet temperature with net shaft power for a single-spool gas turbine engine. 


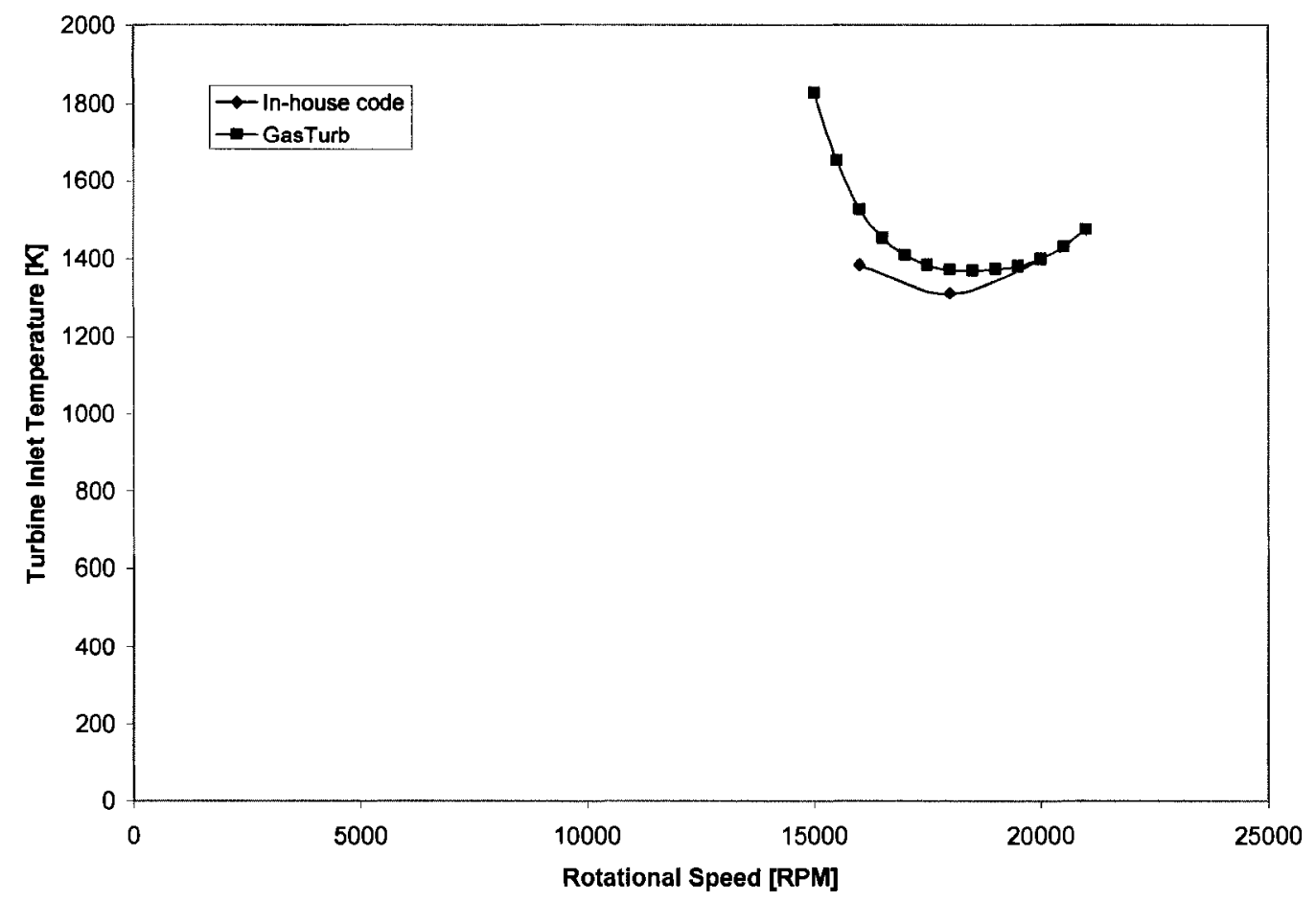

Figure 4.28 Variation of turbine inlet temperature with rotational speed for a single spool gas turbine engine.

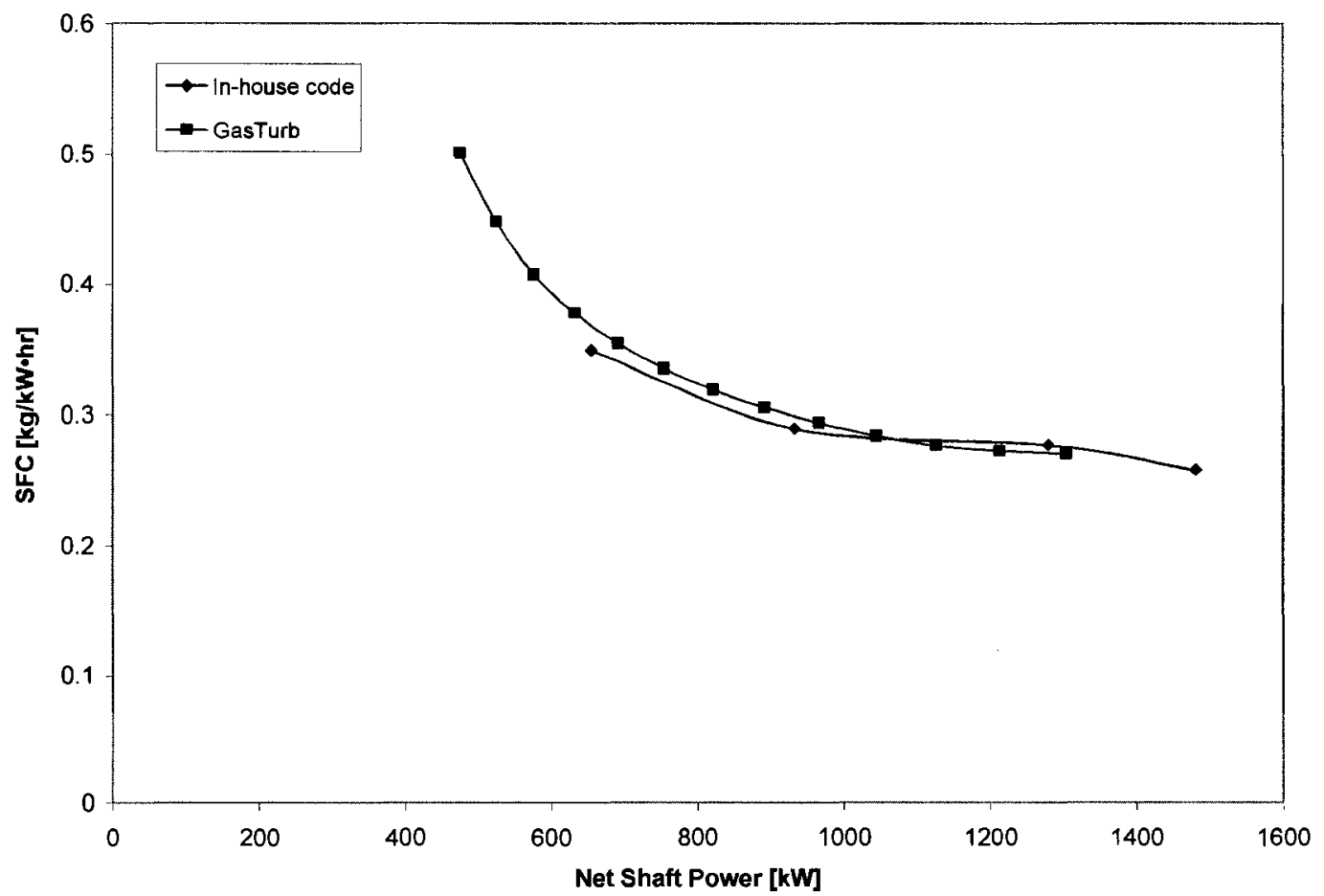

Figure 4.29 Variation of specific fuel consumption with net shaft power for a single spool gas turbine engine. 
Comparison of Figures 4.27 through 4.29 show good agreement between the GasTurb $^{\mathrm{TM}}$ analysis and the in-house code created; all the general trends are replicated by the in-house code. Shaft power appears to be over-predicted by the in-house code (as indicated by Figure 4.27), however this can be attributed to differences in the equilibrium running line, as well as possible differences in the specific heat capacity used in the calculation of compressor and turbine work.

Figure 4.28 shows good matching between the turbine inlet temperatures at, and around, the design speed, however the prediction accuracy degrades as the rotational speed decreases.

Figure 4.29 shows excellent agreement with the specific fuel consumption as predicted by GasTurb ${ }^{\mathrm{TM}}$ and the in-house code. SFC is predicted within 5 percent difference of the actual value over the operating range. Furthermore, of the parameters tested against GasTurb ${ }^{\mathrm{TM}}$, the in-house model predicts the performance well, and can be used to determine the overall engine performance of the impeller based upon the maps generated by the two-zone model.

\subsection{Performance Modelling}

Overall performance modelling of gas turbine engines utilizing centrifugal compressors was the ultimate goal of the project undertaken. While this was the goal, the actual calculations comprise a small section of the research performed; to establish these 
results, confidence needed to be generated in the two-zone model, the low speed extrapolation technique and the component matching calculations (refer to sections 4.1 through 4.3).

Figures 4.30 through 4.32 show the performance of three engines using the Eckhardt $\mathrm{O}$ impeller, the Eckhardt $\mathrm{A}$ impeller and the impeller described by Came and Herbert (1980) using both the actual impeller maps obtained from the literature and the maps as determined using the two-zone model.

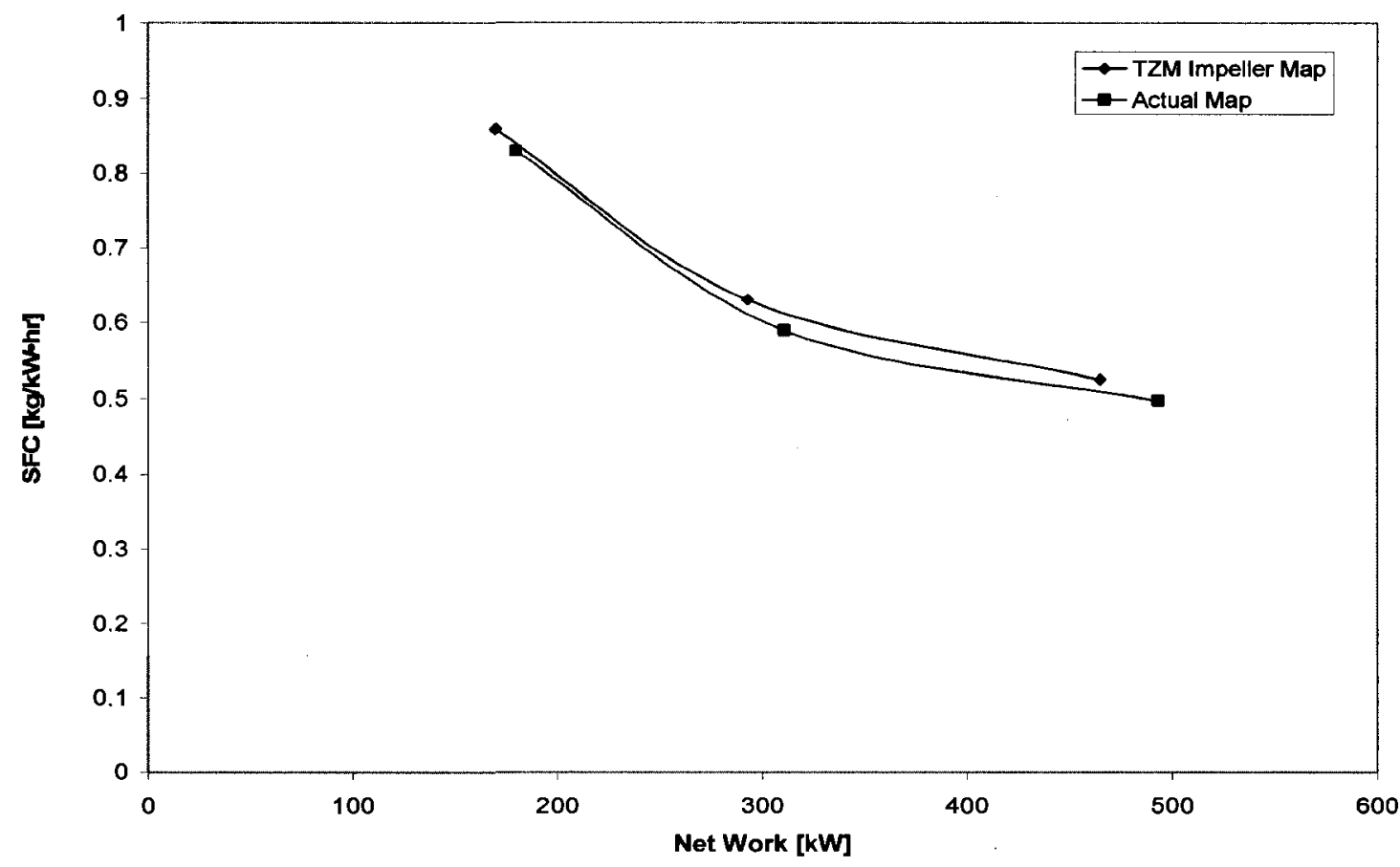

Figure 4.30 Performance of Eckhardt impeller $O$ using both the actual impeller map and the TZM impeller map. 


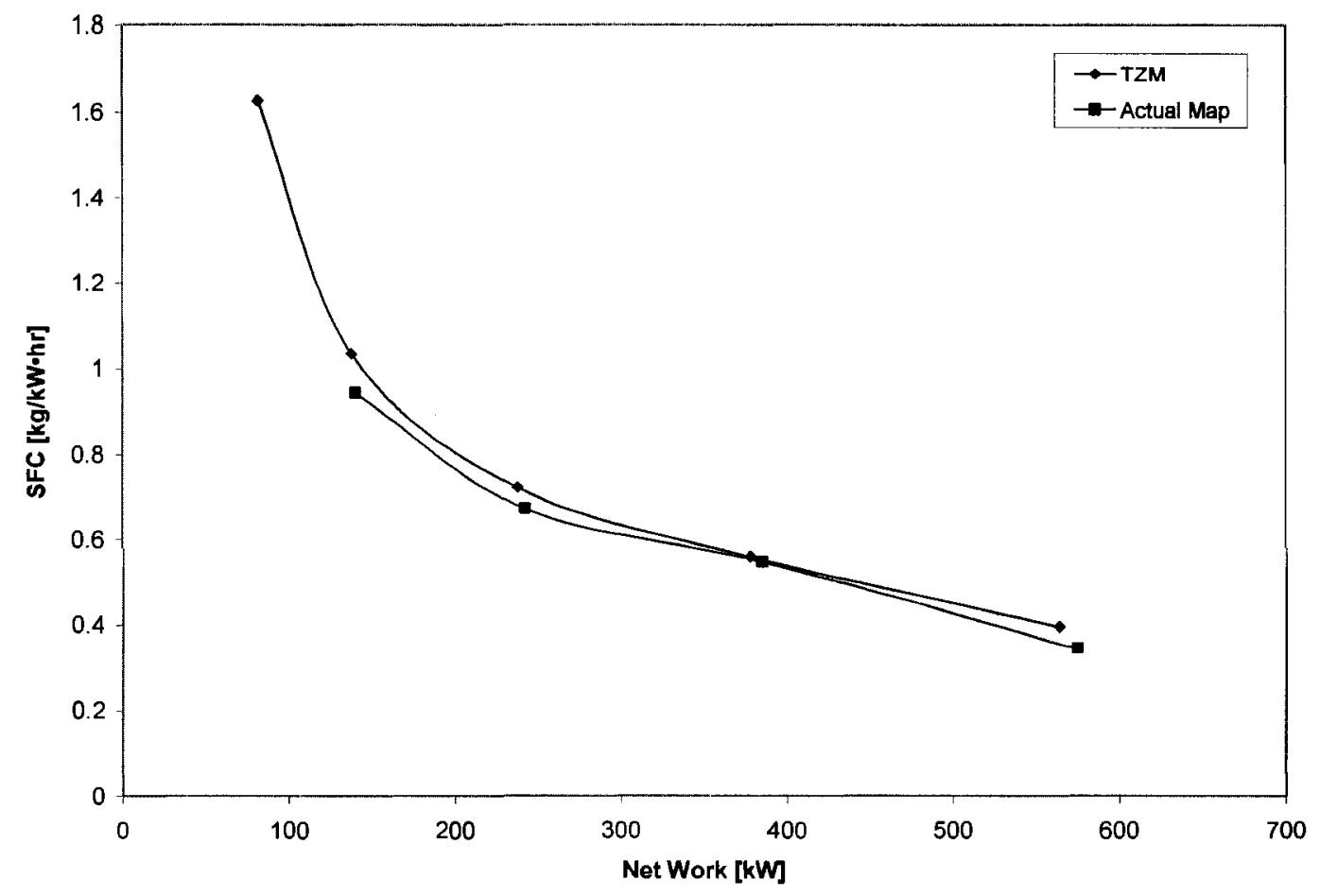

Figure 4.31 Performance of Eckhardt impeller A using both the actual impeller map and the TZM impeller map.

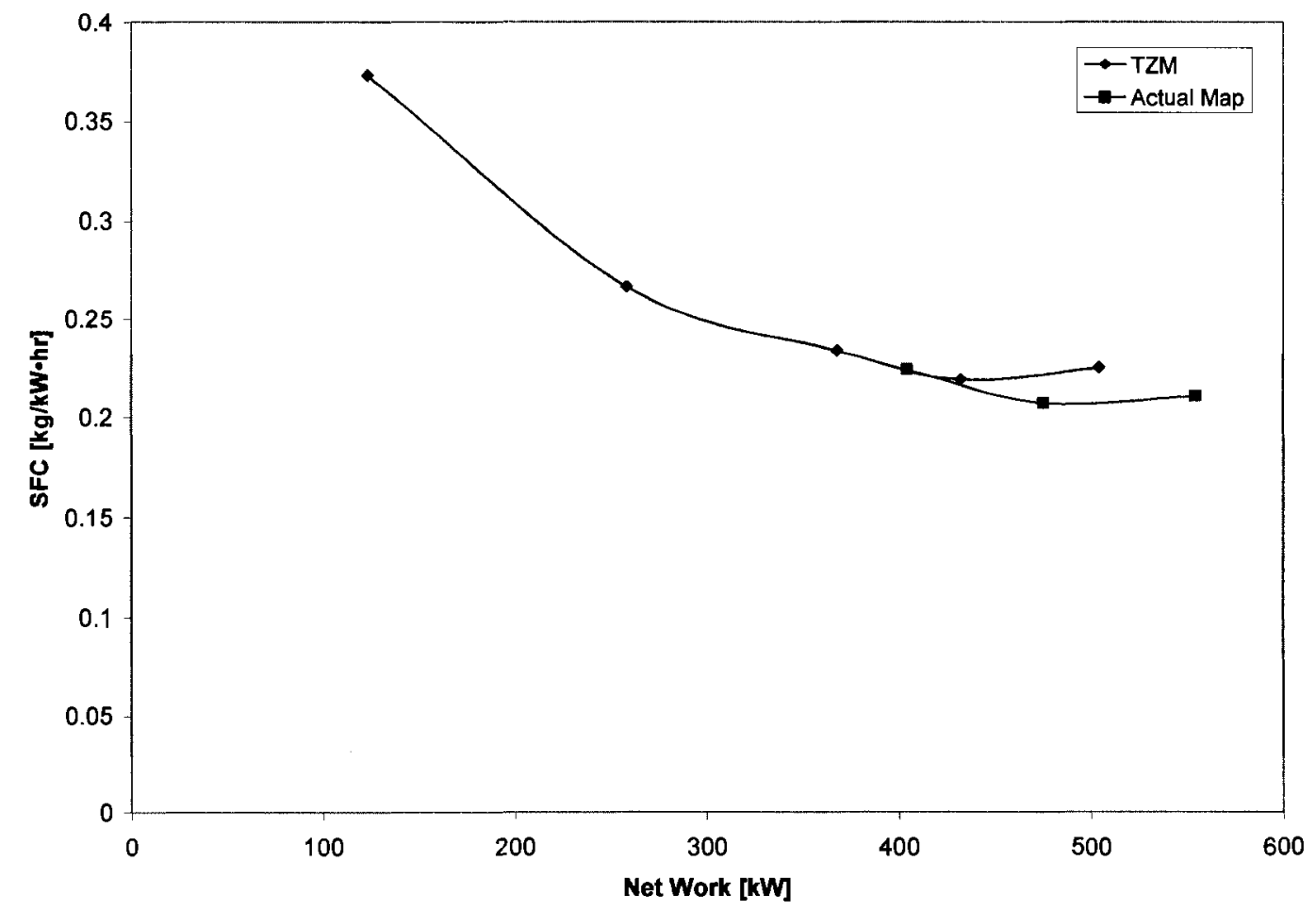

Figure 4.32 Performance of the impeller described by Came and Herbert (1980) using both the actual impeller map and the TZM impeller map. 
All figures show good agreement between the actual maps and the map determined by the two-zone model. Of interest is Figure 4.32; this impeller shows the greatest difference between the actual map and the map calculated by the two-zone model. The map as calculated by the two-zone model shows a much wider range for calculated SFC. Referring to Figure 4.15 , it can be seen that the two-zone model slightly over-predicts the pressure ratio at the low spool speed range and under-predicts isentropic efficiency at all rotational speeds. This combination allows for more operating points to be calculated by the component matching algorithm as the required amount of shaft power can be delivered on the lower characteristics.

Figure 4.33 shows the specific fuel consumption based upon the actual impeller maps for the three impellers tested compared with the specific fuel consumption as calculated by the composite model utilizing the two-zone model, the low speed extrapolation technique, the component matching technique and the performance prediction algorithm. 


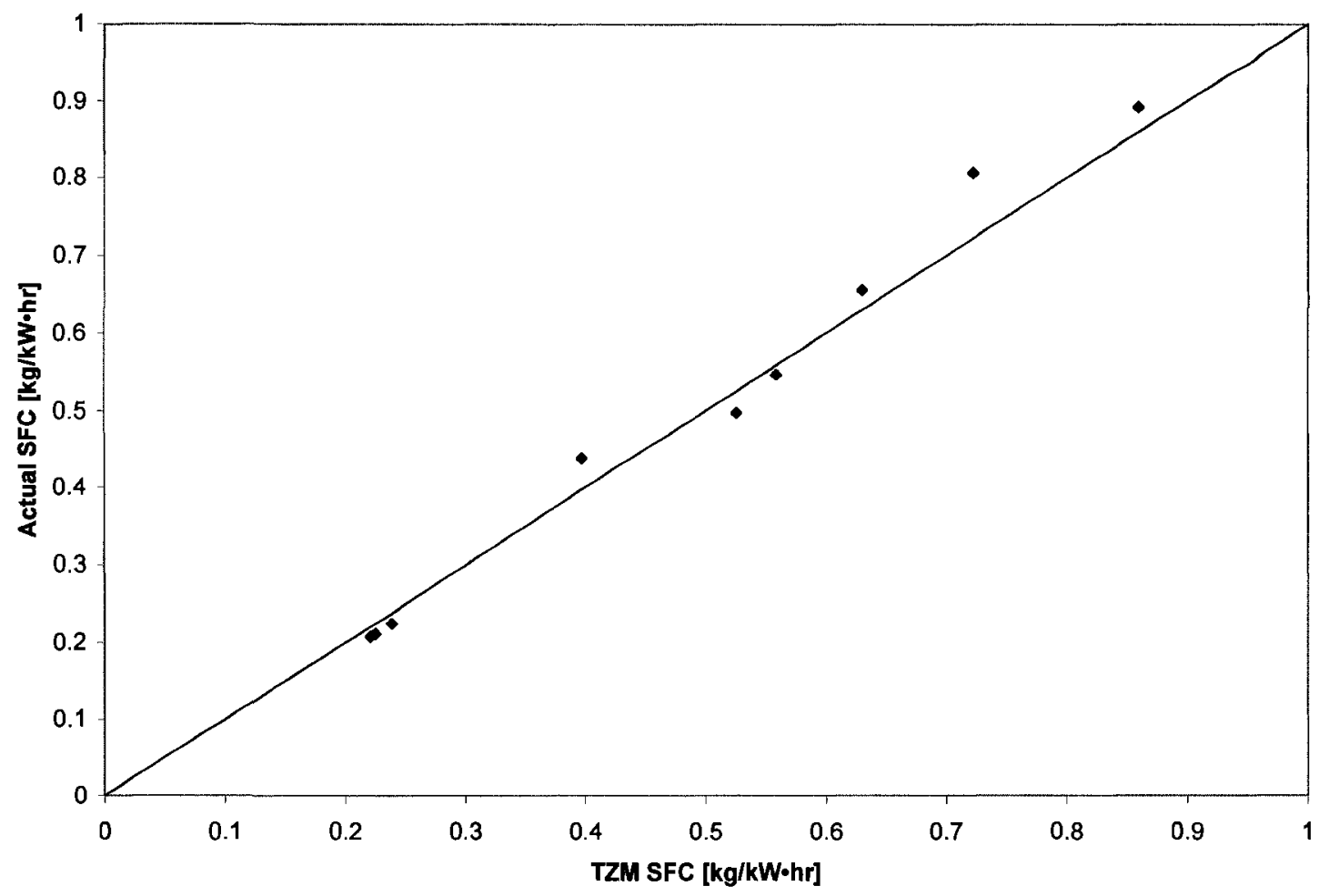

Figure 4.33 Comparison of SFC calculations based upon the actual maps and the maps obtained by the TZM.

Figure 4.33 is particularly interesting as it shows the ability of the overall design tool developed in-house to simulate the performance of gas turbine over a wide variety of operating conditions. Specific fuel consumption is well predicted by the two-zone model when compared to the values determined from the actual impeller maps.

This concludes the validation of the tool developed for the off-design performance prediction of gas turbine engines employing centrifugal impellers. Results have been presented indicating: 1) the accuracy of the two-zone model in determining the performance of centrifugal impellers, 2) the validity of the low speed extrapolation technique in determining the start-up region, 3) the accuracy of the in-house model in 
determining the part-load performance of a single-spool gas turbine engine and 4) the performance the in-house performance prediction algorithm gives for impeller maps generated by the two-zone model when compared to the actual maps obtained from the literature. Recommended future work and conclusions are presented next. 


\section{Conclusions and Recommendations}

This final chapter will outline the accomplishments presented in this thesis regarding the off-design analysis tool developed. The tool was successfully validated against several existing centrifugal impellers using their associated impeller maps; the tool itself gives a good indication of the behaviour of centrifugal impellers at off-design conditions.

Several recommendations are also presented which are based on the experience in the development of off-design tool. Furthermore, comments are given regarding the applicability of the model in the preliminary design phase.

\subsection{Conclusions}

A complete set of equations to establish the full centrifugal compressor map with an associated vaneless diffuser has been presented. Furthermore, models to extrapolate the map to the low speed region have been presented. Finally, models showing the component matching and performance prediction of a single-spool gas turbine engine have been introduced. From these models, a preliminary design tool was created in MATLAB to automate the off-design analysis process. Several steps of tool validation were undertaken. To validate the two-zone model tool, compressor maps were generated from impeller geometry and compared to the actual maps obtained in the literature. Validation of the component matching and performance prediction tools was performed 
by determining the ability of the in-house model to estimate the running line of a compressor map taken from GasTurb ${ }^{\mathrm{TM}}$. The running line was then compared with that generated by GasTurb ${ }^{\mathrm{TM}}$ itself, a well known gas turbine performance prediction software package. The performance prediction algorithm was then used to determine gas turbine engine efficiency by generating plots of net work output versus specific fuel consumption.

It was found that with appropriate effectiveness values, $\eta_{a}$ and $\eta_{b}$, two-zone modelling can predict centrifugal compressor characteristics well (within 2 percent of actual impeller values). Furthermore, simple component matching calculations can approximate gas turbine engine performance on the same order of accuracy as

commercial software packages such as GasTurb ${ }^{\mathrm{TM}}$. Finally, the coupling of two-zone models with low speed extrapolation methods, component matching calculations and performance estimation calculations, gas turbine engines using centrifugal compressors can be predicted well as required for preliminary design calculations.

\subsection{Recommendations}

Despite the ability of the off-design tool to predict gas turbine engine performance, several changes and improvements can be made to the code which could greatly improve its operation. Recommended actions include: 
- Development of appropriate correlations to predict inlet and passage effectiveness, $\eta_{a}$ and $\eta_{b}$, both at the design point and their variation with flow conditions at other rotational speeds and mass flow rates.

- Investigations into the effect of inlet and passage effectiveness, $\eta_{a}$ and $\eta_{b}$, as well as wake mass flow fraction values on two-zone model performance.

- At present, the in-house code runs the two-zone model separately from the remainder of the modelling algorithms due to the requirement for the user to manually determine the relevant two-zone model data. Automation and coupling of the two-zone model to the low speed extrapolation, component matching and performance modelling algorithms would improve use and reduce possible user error.

- Addition of an off-design turbine analysis package as well as appropriate component matching calculations.

- Addition of a free power turbine and jet engine component matching calculations.

- Addition of a vaned diffuser performance algorithm.

- Integration of the off-design code to a larger general preliminary turbomachinery design software package.

- Incorporation of the off-design code with a graphical user interface.

- Improvement of the beta grid development 


\section{References}

Anonymous, 2007a, Concepts NREC - Products - Software - Agile Software - AxCent, http://www.conceptsnrec.com/products/agile_axcent.htm, Accessed Nov. 13, 2007.

Anonymous, 2007b, GasTurb ${ }^{\mathrm{TM}}$ - Products, http://www.gasturb.de/Products/products.html, Accessed Oct. 17, 2007.

Attia, M.S., Schobeiri M.T., A New Method for the Prediction of Compressor Performance Maps Using One-Dimensional Row-by-Row Analysis, Proceedings of the International Gas Turbine and Aeroengine Congress and Exposition 1995, 95-GT-434.

Aungier, R.H. Centrifugal Compressors: A Strategy for Design and Analysis, The American Society for Mechanical Engineers, 2000.

Boyer, K. M., An Improved Streamline Curvature Approach for Off-Design Analysis of Transonic Compression Systems, Ph. D Thesis, Virginia Polytechnic Institute and State University, Dept. of Mechanical Engineering, 2001.

Came, P.M. and Herbert M.V., "Design and Experimental Performance of some High Pressure Ratio Centrifugal Compressors", AGARD CP-282, pp.15.1-15.31, 1980.

Cerri, G., Salvini, C., Procacci, R., Rispoli, F., Fouling and Air Bleed Extracted Flow Influence on Compressor Performance, Proceedings of the ASME Turbo Expo 1993, 93GT-366, 1993.

Daily, J.W. and Nece, R.E., Chamber dimension effects on induced flow and frictional resistance of enclosed rotating disks, Transactions of the ASME, Journal of Basic Engineering, 82, pg. 217-232, 1960.

Daneshar, H. and Shaalan, M.R.A., The Off-Design Analysis of Flow in Axial Compressors, Aeronautical Research Council, C.P. No. 1234, Mar. 1971.

Davis, W.R., Comparison of Different Forms of the Quasi-Three-Dimensional Radial Equilibrium Equation of Turbomachines, Report No. ME/A 68-1, Carleton University, Ottawa, ON, 1968.

Davis, W.R., Millar, D.J., A Comparison of the Matrix and Streamline Curvature Methods of Axial Flow Turbomachinery Analysis, From a User's Point of View, Transactions of the ASME: Journal of Engineering for Power, 97, pg 549-560, 1975.

Dixon, S.L., Fluid Mechanics and Thermodynamics of Turbomachinery: Fourth Edition, Butterworth-Heinemann Publishing, Burlington, MA, 1998. 
Eckhardt, D., Instantaneous Measurements in the Jet-Wake Discharge Flow of a Centrifugal Compressor Impeller, Transactions of the ASME: Journal of Engineering for Power, Vol. 97, 1975, pp. 337-346.

Eckhardt, D., Flow Field Analysis of Radial and Backswept Centrifugal Compressor Impellers, Part I: Flow Measurements Using a Laser Velocimeter, ASME $25^{\text {th }}$ Annual International Gas Turbine Conference, A80-36101 14-34, pp. 77-86, New Orleans, Louisiana, March 1980.

Gaudet S.R. and Gauthier, J.E.D., A Simple Sub-Idle Component Map Extrapolation Method, Proceedings of the ASME Turbo Expo 2007: Power for Land, Sea and Air, May 14-17, 2007.

Gauthier, J.E.D., MECH 5402: Gas Turbine Performance, Supplementary Course Notes, Department of Mechanical and Aerospace Engineering, Carleton University, 2006.

Gravdahl, J.T., Egeland, Olav, Compressor Surge and Rotating Stall: Modeling and Control, Springer-Verlag, London, UK, 1999.

Hale, A.A., Davis, M.W. Jr. and Kneile, K.R., Turbine Engine Analysis Compressor Code: TEACC - Part I: Technical Approach and Steady Results, AIAA-94-0148, 32 ${ }^{\text {nd }}$ Aerospace Sciences Meeting, Reno, NV, January 1994.

Japikse, D. Assessment of single and two-zone modelling of centrifugal compressors. Studies in component performance: Part 3. ASME Paper 85-GT-73, 1985.

Japikse, D. Centrifugal Compressor Design and Performance, Concepts ETI, Wilder, 1996.

Kong, C., Jayoung, K., Seonghee, K., Myoungchoel, K., Improvement of Performance Simulation Using Component Maps of Aircraft Gas Turbine Obtained from System Identification, AIAA Paper, ISABE-2003-1145, 2003.

Kurzke, J., How to Get Component Maps for Aircraft Gas Turbine Performance Calculations, ASME Paper 96-GT-164, 1996.

Kurzke, J., Riegler, C., A New Compressor Map Scaling Procedure for Preliminary Conceptual Design of Gas Turbines, Proceedings of the ASME IGTI, Turbo Expo 2000, May 8-11, 2000.

Lefebvre, A. H., Gas Turbine Combustion: Second Edition, Taylor and Francis Publishing, Philadelphia, PA, 1996.

McKain, T.F. and Holbrook, G.J., Coordinates for a High Performance 4:1 Pressure Ratio Centrifugal Compressor, NASA CR-204134, 1997. 
Muir, D.E., Axial Flow Compressor Modelling for Engine Health Monitoring Studies, M. Eng. Thesis, Carleton University, Dept. of Mechanical and Aeronautical Engineering, 1988.

Novak, R.A., Streamline Curvature Computing Procedures for Fluid Flow Problems, Transactions of the ASME: Journal of Engineering for Power, 89, pg 478-490, 1967.

Oh, H.W., Yoon, E.S. and Chung, M.K., An Optimum Set of Loss Models for Performance Prediction of Centrifugal Compressors, Proceedings of the Institution of Mechanical Engineers, Part A, Journal of Power and Energy, 211(A4), pg. 331-338, 1997.

Oh, H.W., Yoon, E.S. and Chung, M.K., Systematic two-zone modelling for performance prediction of centrifugal compressors, Proceedings of the Institution of Mechanical Engineers, Part A, Journal of Power and Energy, 216, pg. 75-87, 2002.

Pacheidis, V., Pilidis, P., Templalexis, I., Alexander, T., Kotsiopoulos, P. Prediction of Engine Performance Under Compressor Inlet Flow Distortion Using Streamline Curvature, Proceedings of ASME Turbo Expo 2006: Power for Land, Sea and Air, May 8-11, 2006.

Pelton, R.J., One Dimensional Radial Flow Turbomachinery Modeling, M.A. Sc. Thesis, Department of Mechanical Engineering, Brigham Young University, 2007.

Roberts, S.K., Effects of Fluid Properties on the Aerodynamic Performance of Turbomachinery for Semi-Closed Gas Cycle Turbine Engines Using $\mathrm{O}_{2} / \mathrm{CO}_{2}$ Combustion, M.A.Sc. Thesis, Department of Mechanical Engineering, Carleton University, 2001.

Rohne, K.H. and Banzhaf, M. Investigation of the flow at the exit of an unshrouded centrifugal impeller and comparison with the 'classical' jet-wake theory, Transactions of the ASME, Journal of Turbomachinery, 113, pg. 654-659, 1991.

Saravanamuttoo, H.I.H., Rogers, G.F.C. and Cohen, H. Gas Turbine Theory: Fifth Edition, Pearson Education Limited, 2001.

Senoo, Y., Yamaguchi, M., Nishi, M., A Photographic Study of the Three-Dimensional Flow in a Radial Compressor, Transactions of the ASME: Journal of Engineering for Power, Vol. 88, No. 1, 1966.

Sexton, W.R., A Method to Control Turbofan Engine Starting by Varying Compressor Surge Valve Bleed, Virginia Polytechnic Institute and State University Master of Science Thesis, 2001.

Sjolander, S.A., Fluid Machinery/Turbomachinery, Supplementary Course Notes, Department of Mechanical and Aerospace Engineering, Carleton University, 2006. 
Song, X., Untaroiu, A., Wood, H.G., Allaire, P.E., Throckmorton, A.L., Day, S.W. and Olsen, D.B., Design and Transient Computational Fluid Dynamics Study of a Continuous Axial Flow Ventricular Assist Device, ASAIO Journal, 2004.

Spina, P.R., Gas Turbine Performance Prediction By Using Generalized Performance Curves of Compressor and Turbine Stages, Proceedings of the ASME Turbo Expo 2002, GT-2002-30275, June 3-6, 2002.

Steinke, R.J., STGSTK: A Computer Code for Predicting Multistage Axial Flow Compressor Performance by a Meanline Stage Stacking Method, NASA Technical paper $2020,1982$.

Stone, A., Effects of Stage Characteristics and Matching on Axial Flow Compressor Performance, ASME Journal of Engineering for Power, pg 127, 1958.

Whitfield, A. Slip factor of a centrifugal compressor and its variation with flow rate. Proceedings of the Institution of Mechanical Engineers, Thermodynamics and Fluid Mechanics Research Group, Fluid Machinery Group, 188, pg. 557-565, 1974.

Whitfield, A., Preliminary Design and Performance Prediction Techniques for Centrifugal Compressors, Proceedings of the Institution of Mechanical Engineers, Part A: Journal of Power and Energy, Vol. 204, 1990, pp. 131-144.

Turns, S.R., An Introduction to Combustion-Concepts and Applications: Second Edition, McGraw-Hill Publishing, 2000. 


\section{Appendix A - Centrifugal Compressor Map Prediction Tool Source Code}

The following appendix outlines the source code developed in MATLAB describing the two-zone model. Equations outlined in chapter three are explicitly used here in conjunction with several iterative methods.

\section{Main.m}

o The following m-file script holds the main impeller two-zone model

o algorithm. It reads in the design point data and geometry from a user

o defined ASCII file, asks the user for information regarding the impeller

o map such as the range of mass flow rates to generate data and the range

\% and values of speed characteristics to generate. Furthermore, each part

of the two-zone model is called and returns data pertinent to that

o particular part. Finally, all corrected map data is calculated and o written to output data files.

$\mathrm{ClC}$

clear

o Clear the output data files

dlmwrite('PR.dat', [], 'delimiter', '\t');

dlmwrite('mdot.dat', [], 'delimiter', '\t');

dlmwrite('Nvalues.dat', [], 'delimiter', ' $\backslash t$ ');

dlmwrite('filename.dat', [], 'delimiter', '\t');

dlmwrite('efficiency.dat', [], 'delimiter', 'ไt');

warning off all

o Ask user for the input data file

filename=input('Please enter the filename containing the compressor geometry: ', ' $\left.S^{\prime}\right)$;

z Reads appropriate data into their respective variables compgeom $=$ dlmread (filename);

$\mathrm{P01}=$ compgeom $(1) * 1000$;

$\mathrm{TO1}=$ compgeom (2);

mdot des $=$ compgeom (3);

Ndes $=$ compgeom $(4)$;

$\mathrm{r} 1 \mathrm{~h}=$ compgeom $(5) / 1000$;

rlt=compgeom $(6) / 1000$;

r2=compgeom (7)/1000; 


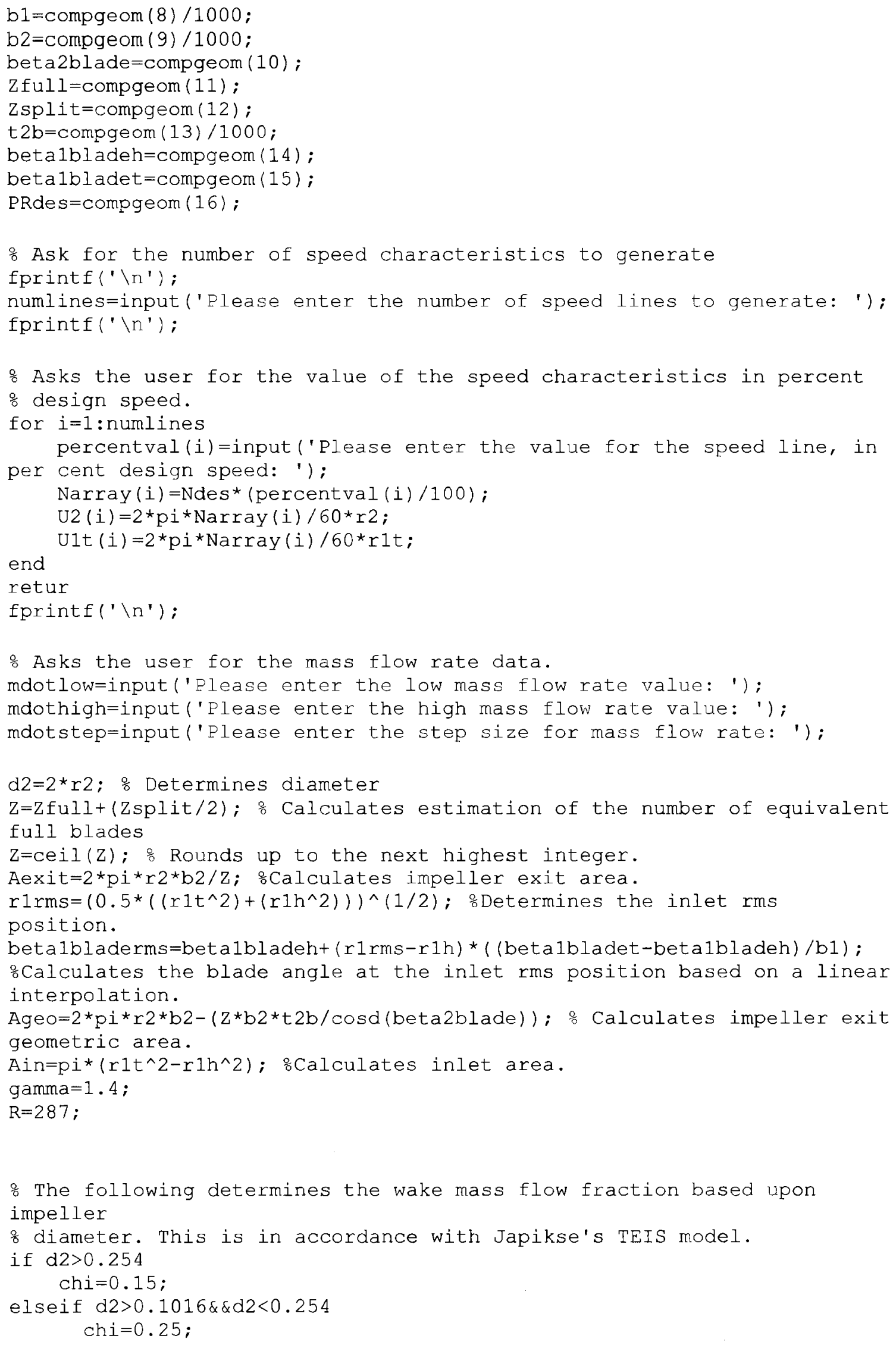




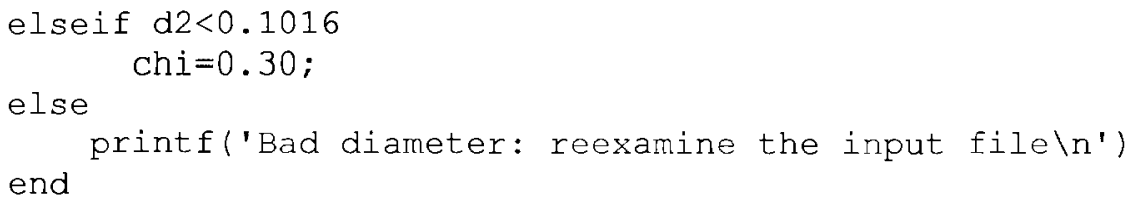

U2des $=2{ }^{*} \mathrm{pi}$ Ndes $/ 60{ }^{*} \mathrm{r} 2$; \% Calculates the design point impeller outlet tangential velocity.

U1tdes $=2{ }^{*} \mathrm{p} i{ }^{*}$ Ndes $/ 60^{*}$ r1t; $\%$ Calculates the design point impeller RMS

inlet tangential velocity.

eta $A=0.83 ;$ User specified inlet effectiveness.

eta $\mathrm{B}=0.45 ;$ o User specified passage effectiveness.

mdotvals=[mdotlow:mdotstep:mdothigh]; \& Creates the array holding the mass flow rate data

Tref $=288$; 8 Reference temperature.

P01ref $=101300$; $\frac{\text { of }}{\circ}$ Reference pressure.

o The following algorithm is the main section of this m-file script.

All

\% the subsections of the two-zone model are called, their respective

o calculations performed, and output values are then referred back to the

\% main.m m-file for calculations in other algorithms. Inlet, diffusion o ratio, jet zone, wake zone and mixed-out state calculations are performed

o in that order.

for $i=1:$ size (Narray, 2)

for $j=1:$ size(mdotvals, 2)

inletvect=inlet (Ain, mdotvals(j), T01, P01, u1t(i), gamma,

R); $\frac{\circ}{0}$ Calls the inlet zone calculation scheme

Cxl=inletvect (1); : Inlet axial velocity

$\mathrm{T} 1=\mathrm{inletvect}(2) ;$ o Inlet static temperature

M1=inletvect (3); Inlet Mach number

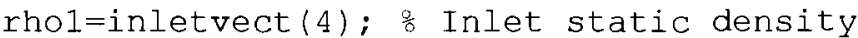

w1t=inletvect (5); $\frac{\circ}{0}$ Inlet tip relative velocity

betalt $(j)=$ inletvect $(6)$; $\frac{0}{\partial}$ Inlet relative flow angle

$\mathrm{P} 1=$ inletvect $(7) ;$ o Inlet static pressure

P01rel=inletvect (8); O Inlet relative stagnation pressure

diffvect=diffusion(Ain, Z, Aexit, etaA, etaB, beta1t(j),

beta1bladerms, W1t); 8 Calls the diffusion ratio calculation scheme

$W 2 j(j)=\operatorname{diffvect}(1)$; o Jet outlet relative velocity

$\operatorname{DR}(j)=\operatorname{diffvect}(2) ;$ Diffusion ratio

jetvect=jetcalc (mdotvals $(j)$, gamma, R, T1, Cx1, U1t(i),

$\mathrm{U} 2(i), W 2 j(j)$, chi, P1, Ageo, r2, beta2blade, b2, Z); $\frac{\circ}{\partial}$ Calls the jet zone calculation scheme

hT=jetvect (1); \& Static enthalpy

$\mathrm{T} 2 j=j$ etvect $(2)$; $\frac{\mathrm{g}}{\mathrm{o}}$ Jet exit static temperature

P2j=jetvect (3); $\%$ Jet exit static pressure

rho $2 j=j e t v e c t(4)$; $\frac{\circ}{\sigma}$ Jet exit static density 
epsilon=jetvect(5); o Wake are fraction

beta $2 j(j)=j$ etvect $(6)$; $\frac{\circ}{\circ}$ Jet exit relative flow angle

Cr $2 j=j$ etvect $(7)$; $\frac{\circ}{0}$ Jet exit meridional velocity

Ctheta2j=jetvect $(8)$; o Jet exit tangential velocity

$\mathrm{C} 2 \mathrm{j}=$ jetvect $(9)$; $\frac{\circ}{8}$ Jet exit absoute velocity

alpha2j=jetvect (10); \% Jet absolute exit angle

$T 02 j=j$ etvect (11); $\frac{\circ}{5}$ Jet exit stagnation temperature

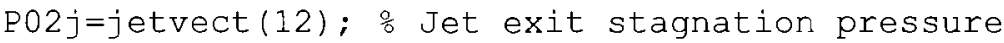

wakevect=wakecalc(hT, U2(i), T2j, rho2j, mdotvals(j), Cr2j,

epsilon, P2j, Ageo, beta2blade, gamma, R); $\frac{\circ}{\circ}$ Calls the wake zone

calculation scheme

P02w=wakevect (1); 号 Wake exit stagnation pressure

T02W=wakevect $(2)$; $\frac{\circ}{3}$ Wake exit stagnation temperature

$\mathrm{C} 2 \mathrm{w}=$ wakevect (3); $\frac{\circ}{\mathrm{o}}$ Wake exit absolute velocity

Ctheta2w=wakevect (4); $\frac{\circ}{0}$ wake exit tangential velocity

Cr2 $\mathrm{w}=$ wakevect $(5)$; $\frac{\circ}{5}$ Wake exit meridional velocity

$\mathrm{T} 2 \mathrm{~W}=$ wakevect $(6) ;$ o wake exit static temperature

rho2w=wakevect (7); $\frac{o}{8}$ Wake exit static density

W2W=wakevect $(8) ;$ o wake exit relative velocity

mixvect $=$ mixcalc $(\mathrm{chi}$, Ctheta2j, Ctheta2w, T02j, T02w,

mdotvals(j), r2, b2, gamma, R, Ageo, P2j, Cr2j, Cr2w, beta2j(j),

beta2blade, $\mathrm{U} 2(i), w 2 j(j), w 2 w, w 1 t, r 1 t, z$, rhol, $\mathrm{Cx} 1, \mathrm{rlh}) ;$ \% Calls

the mixed-out static calculation scheme

P02mix=mixvect(1); $\frac{\circ}{\circ}$ Mixed-out stagnation pretture

T02mix=mixvect (2); \% Mixed-out stagnation temperature

$\mathrm{P} 2 \mathrm{~m}=\mathrm{mixvect}(3)$; $\frac{\circ}{\circ}$ Mixed-out static pressure

T2m=mixvect $(4)$; $\frac{\circ}{6}$ Mixed-out static temperature

rho2m=mixvect (5); \& Mixed-out static density

$\mathrm{c} 2 \mathrm{~m}=\mathrm{mixvect}(6) ;$ o Mixed-out absolute velocity

beta $2 \mathrm{~m}(j)=$ mixvect $(7)$; $\frac{\circ}{\circ}$ Mixed-out relative flow angle

$\operatorname{PRmap}(j, i)=\mathrm{P} 02 \mathrm{mix} / \mathrm{P01}$; Calculates pressure ratio

efficiency $(j, i)=(T 02 j-T 01) /(T 02 \mathrm{mix}-\mathrm{T} 01)$; $\frac{\circ}{\circ}$ Calculates map

efficiency

end

end

o The following three lines calculate the output corrected mass flow values

$\frac{\circ}{\partial}$ and output corrected rotational speed values. These are based upon the

$\therefore$ reference pressure and temperature as specified above.

mdotcorr=mdotvals* ((T01/Tref $\left.)^{\wedge} 0.5\right) /($ P01/P01ref $)$;

Nout=Narray $/\left((\text { T01/Tref })^{\wedge} 0.5\right)$;

Nout $=$ Nout . ' ;

o The following line initialize the output files and write the map output raw

o data. The user then has to manually clean the data and supply to the rest of

\% the algorithm, starting with the beta grid definition. 


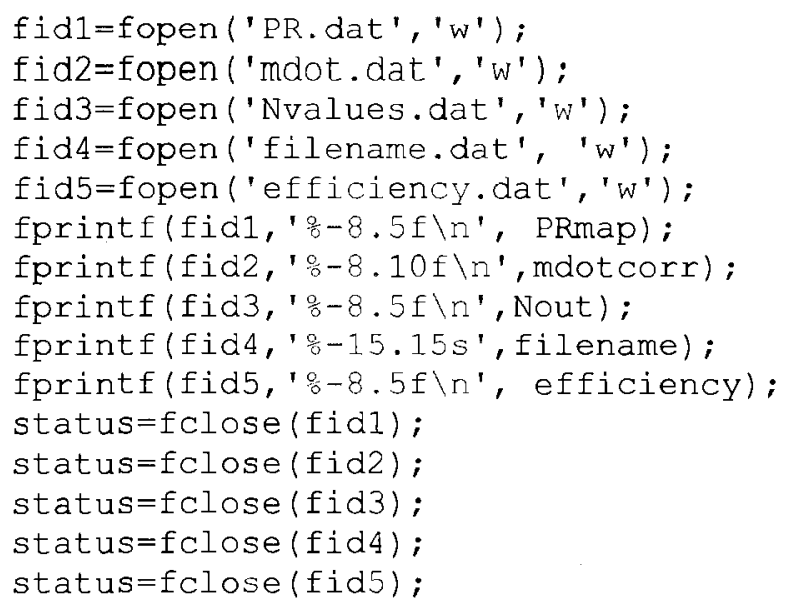

\section{Inlet.m}

o The following m-file performs the calculations necessary in the o determination of the inlet zone quantities. Since only stagnation o properties are known at the outset, it is necessary to perform an o iterative analysis at the inlet. Inputs are inlet area, mass flow rate, \% stagnation temperature, stagnation pressure, inlet tangential speed, o specific heat ratio and universal gas constant. The output files are the

o supplied back to the main.m m-file.

function output=inlet (Ain, mdot, T01, P01, U1t, gamma, R)

maxiter $=0$; $\frac{\circ}{\partial}$ Loop counter

rho01=P01/(R*T01); 品 Inlet stagnation density

rholguess=rho01; Guess for the iterative loop

while maxiter $<50$

CxI=mdot $/($ Ain*rholguess $) ;$ o Inlet axial velocity $\mathrm{T} 1=\mathrm{T} 01-\left(1 /\left(2^{\star}\right.\right.$ gamma ${ }^{\star} \mathrm{R} /($ gamma -1$\left.\left.)\right)\right){ }^{\star} \mathrm{Cx} 1^{\wedge} 2$; $\%$ Inlet static temperature $\mathrm{M} 1=\mathrm{Cx} 1 /\left(\left(\text { gamma } \mathrm{R}^{\star} \mathrm{T} 1\right)^{\wedge}(1 / 2)\right) ;$ 응 Inlet Mach number rholnew $=r h o 01 /\left(\left(1+\left((\text { gamma-1)/2 })^{\star M} 1^{\wedge} 2\right)^{\wedge}(1 /(\right.\right.$ gamma-1 $\left.))\right)$; 응 New approximation to inlet static density

conv=rholguess-rholnew; $\frac{\circ}{\circ}$ Loop convergence parameter

of The following if statement checks to see if the inlet static density

$\%$ has converged. If it has (within a prescribed tolerance), the loop is

\% exited and further quantities are calculated. If not, a new guess of

o the inlet static density is supplied. 


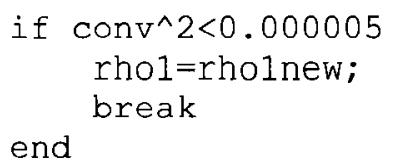

end

$W 1 t=\left(\left(\mathrm{Cx} 1^{\wedge} 2\right)+\left(\mathrm{U} 1 \mathrm{t}^{\wedge} 2\right)\right)^{\wedge}(1 / 2)$; o Inlet tip relative velocity from velocity triangles

beta1t=atand $(\mathrm{U} 1 \mathrm{t} / \mathrm{Cx} 1)$; 8 Inlet tip relative flow angle

$\mathrm{P} 1=\mathrm{P} 01 /((1+(($ gamma-1) $) / 2) \star \mathrm{M} 1 \wedge 2) \wedge($ gamma $/($ gamma-1 $)))$; $\frac{\circ}{\circ}$ Inlet static pressure

M1rel $=W 1 t /\left(\left(\text { gamma }{ }^{\star} R^{\star} T 1\right)^{\wedge}(1 / 2)\right)$; o Inlet relative Mach number P01rel $=\mathrm{P} 1 *((1+(($ gamma -1$) / 2) * \mathrm{M} 1 \mathrm{rel} \wedge 2) \wedge($ gamma $/($ gamma -1$)))$; O Inlet relative stagnation pressure

output $=[\mathrm{CX} 1, \mathrm{~T} 1, \mathrm{M} 1$, rhol, W1t, betalt, P1, P01rel]; o Constructs the output vector and returns quantities to main.m

\section{Diffusion.m}

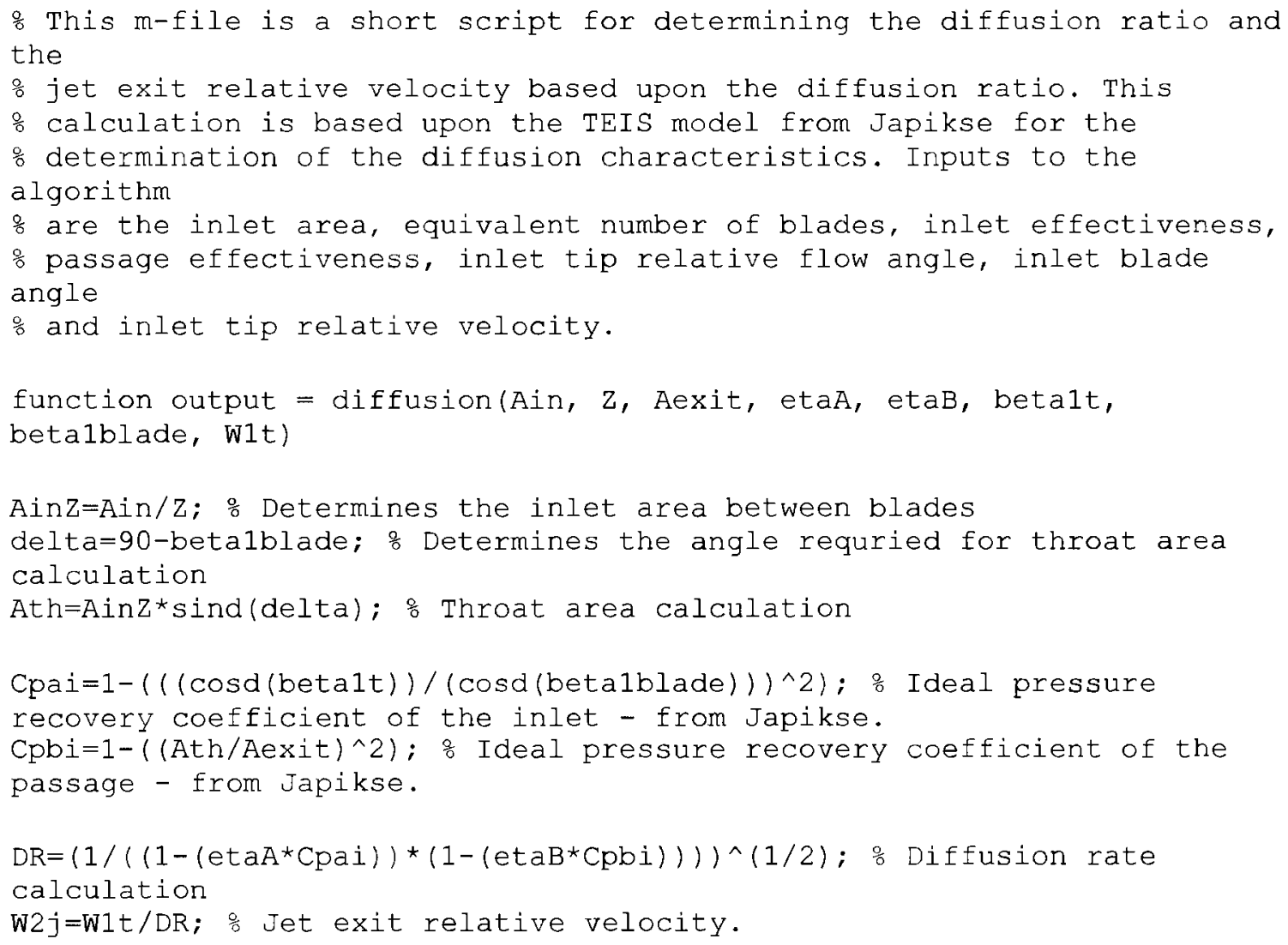




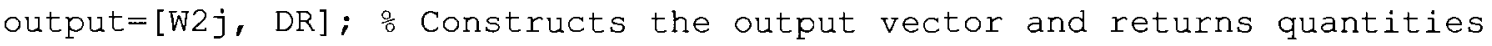
to main.m

\section{Jetcalc.m}

을 The following m-file describes the jet-zone calculation process. This o method is blended from theory presented by oh et al. (2002) and Japikse

\% (1985) based upon two different sets of equations describing the twozone

o model. This process, unlike oh et al., does not requrie any iterative o solution of the wake mass flow fraction, but includes some of the newer

o slip correlations employed by oh et al. (2002) for estimation of the o outlet state. Required inputs are: mass flow rate, specific heat ratio,

\% universal gas constant, inlet static temperature, inlet axial velocity,

o inlet tip tangential velocity, outlet tip tangential velocity, outlet jet

o relative velocity, wake mass flow fraction, inlet static pressure, o geometric area, outlet radius, blade angle, outlet blade height and the

o number of equivalent blades.

function output $=$ jetcalc (mdot, gamma, $R, T 1, \mathrm{Cx} 1, \mathrm{U} 1 \mathrm{t}, \mathrm{U} 2, \mathrm{w} 2 \mathrm{j}$, chi, P1, Ageo, r2, beta2blade, b2, z)

$\mathrm{hT}=($ gamma $* \mathrm{R} /($ gamma -1$)) * \mathrm{~T} 1+\left(\left(\mathrm{Cx} 1^{\wedge} 2\right) / 2\right) ; \%$ Calcualtes the total enthalpy $\mathrm{h} 2 \mathrm{j}=\mathrm{hT}-\left(\left(\mathrm{W} 2 \mathrm{j}^{\wedge} 2\right) / 2\right)+\left(\left(\mathrm{U} 2^{\wedge} 2\right) / 2\right)$; outlet jet enthalpy

$\mathrm{T} 2 \mathrm{j}=\mathrm{h} 2 \mathrm{j} /\left(\right.$ gamma $\mathrm{R} /\left(\right.$ gamma-1)); $\frac{\text { o }}{\mathrm{o}}$ outlet jet static temperature $\mathrm{P} 2 \mathrm{j}=\mathrm{P} 1^{\star}\left((\mathrm{T} 2 \mathrm{j} / \mathrm{T} 1)^{\wedge}\left(\right.\right.$ gamma $/\left(\right.$ gamma-1))); $\frac{\circ}{\partial}$ Outlet jet static pressure rho $2 j=\mathrm{P} 2 \mathrm{j} /\left(\mathrm{R}^{\star} \mathrm{T} 2 \mathrm{j}\right)$; o outlet jet static density

$f j=5.73 e-\sigma^{\star}\left((90-\text { beta2blade })^{\wedge}(5 / 2)\right) ;$ o Slip factor coefficient (Oh et al. (2002))

epsilon= $\operatorname{chi}^{\wedge}(1 / 2)$; o wake area fraction correlation (Japikse (1996)) A $2 j=$ Ageo* (1-epsilon); \& Exit jet area

beta $2 j=($ beta $2 b l a d e / 2)+(0.5 *$ asind $(((2 * U 2 * f j * A 2 j /(W 2 j * r 2 * b 2 * z))+t a n d$ (beta

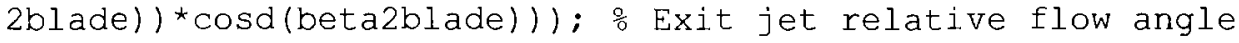

ofter calculation scheme here to include whitfield slip factor to sotermine the outlet relative flow angle.

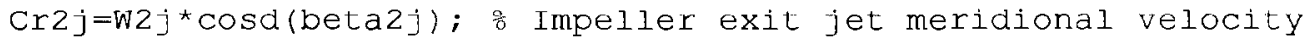
Ctheta $2 j=U 2-\left(W 2 j{ }^{\star}\right.$ sind (beta2j)); $\frac{\circ}{\partial}$ Impeller exit tangential velocity $\mathrm{C} 2 \mathrm{j}^{\prime}=\left(\left(\mathrm{Cr} 2 \mathrm{j}^{\wedge} 2\right)+\left(\mathrm{Ctheta} 2 \mathrm{j}^{\wedge} 2\right)\right)^{\wedge}(1 / 2)$; 응 Impeller exit absolute velocity alpha2j=atand (Ctheta2j/Cr $2 j)$; o Impeller exit absolute flow angle $\mathrm{T0} 2 \mathrm{j}=\mathrm{T} 2 \mathrm{j}+\left(\left(\mathrm{C} 2 \mathrm{j}^{\wedge} 2\right) /\left(2^{\star} \mathrm{gamma}{ }^{\star} \mathrm{R} /(\right.\right.$ gamma-1) $\left.)\right)$; o Jet exit stagnation temperature $\mathrm{P02j}=\mathrm{P} 2 j^{\star}((\mathrm{TO} 2 \mathrm{j} / \mathrm{T} 2 \mathrm{j}) \wedge($ gamma/(gamma-1))); 8 Jet exit stagnation pressure 
output $=[h T, T 2 j$, P2j, rho2j, epsilon, beta2j, Cr2j, ctheta2j, c2j, alpha2j, T02j, P02j]; o Constucts the output vector and returns necessary quantities to main.m.

\section{Wakecalc.m}

\% The following m-file describes the wake zone calculation process. This

o calculation occurs directly after the determination of the jet zone o quantities. This analysis requires an iterative determination of the wake

o exit static temperature. Closure of the system is faciltitated by the

o specification of the unloaded tip condition. No internal loss

o correlations are required. Input parameters include: total rothalpy, exit

응 impeller tangential velocity, jet exit static temperature, jet exit

o static density, mass flow rate, jet exit meridional absolute velocity,

: wake area fraction, jet exit static pressure, impeller geometric area,

\% blade exit angle, specific heat ratio and the universal gas constant.

function output $=$ wakecalc $(\mathrm{hT}, \mathrm{U} 2, \mathrm{~T} 2 \mathrm{j}, \mathrm{rho} 2 \mathrm{j}$, mdot, $\mathrm{cr} 2 \mathrm{j}$, epsilon, P2j, Ageo, beta2blade, gamma, R)

hT $2 \mathrm{w}=\mathrm{hT} ; \mathrm{o}$ Total enthalpy of the wake

$\mathrm{P} 2 \mathrm{w}=\mathrm{P} 2 \mathrm{j}$; $\frac{O}{5}$ Unloaded tip condition

maxiter=0; $\%$ Loop counter

$\mathrm{T} 2$ wguess $=\mathrm{T} 2 \mathrm{j} ; \mathrm{o}$ Guess for the wake exit static temperature

\% The following iterative loop attempts to determine the wake exit static

o temperature through the calculation of an updated estimation of the o static

\% enthalpy of the wake. Once the static temperature has converged,

$\frac{o}{\circ}$ further quantities can be established.

while maxiter $<200$

rho2wake $=\mathrm{P} 2 \mathrm{w} /\left(\mathrm{R}^{\star} \mathrm{T} 2 \mathrm{wguess}\right) ; \quad$ wake exit static density

$\mathrm{Cr} 2 \mathrm{w}=\left((\right.$ mdot $/$ Ageo $)-\left(\operatorname{rho} 2 j{ }^{\star} \operatorname{Cr} 2 j \star(1-\right.$ epsilon $\left.\left.)\right)\right) /\left(\right.$ rho 2 wake ${ }^{\star}$ epsilon $) ; \frac{\circ}{0}$

Wake exit meridional velocity

W2 $w=\operatorname{Cr} 2 \mathrm{w} / \mathrm{cosd}$ (beta2blade); $\mathrm{o}$ wake relative velocity

$h 2 w=h T 2 w-\left(\left(w_{2} w^{\wedge} 2\right) / 2\right)+\left(\left(\mathrm{U} 2^{\wedge} 2\right) / 2\right)$; $\frac{\circ}{\partial}$ Static enthalpy of the wake

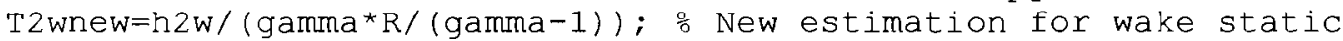
temperature

conv $=\mathrm{T} 2$ wnew-T2wguess; $\frac{\circ}{5}$ Convergence parameter

if $\mathrm{conv}^{\wedge} 2<0.00005$

$\mathrm{T} 2 \mathrm{~W}=\mathrm{T} 2 \mathrm{wnew}$;

break 
end

$\mathrm{T} 2$ wguess $=\mathrm{T} 2$ wnew;

maxiter=maxiter +1 ; $\frac{\circ}{8}$ Loop counter

end

Ctheta $2 \mathrm{w}=\mathrm{U} 2-\mathrm{Cr} 2 \mathrm{w}^{\star}$ tand (beta2blade); $\frac{\circ}{5}$ wake exit tangential absolute velocity

$\mathrm{C} 2 \mathrm{w}=\left(\left(\mathrm{Ctheta} 2 \mathrm{w}^{\wedge} 2\right)+\left(\mathrm{Cr} 2 \mathrm{w}^{\wedge} 2\right)\right)^{\wedge}(1 / 2) ;$ : Wake exit absolute velocity $\mathrm{T} 02 \mathrm{w}=\mathrm{T} 2 \mathrm{w}+\left(\left(\mathrm{C} 2 \mathrm{w}^{\wedge} 2\right) /(2 *\right.$ gamma* $\mathrm{R} /($ gamma-l $\left.))\right) ;$ o wake exit stagnation temperature

$\mathrm{P} 02 \mathrm{~W}=\mathrm{P} 2 \mathrm{~W}^{*}\left((\mathrm{~T} 02 \mathrm{~W} / \mathrm{T} 2 \mathrm{~W})^{\wedge}(\right.$ gamma $/($ gamma-l $\left.))\right) ;$ : Wake exit stagnation pressure

output $=[\mathrm{P} 02 \mathrm{w}, \mathrm{T} 02 \mathrm{w}, \mathrm{C} 2 \mathrm{w}, \mathrm{Ctheta} 2 \mathrm{w}, \mathrm{Cr} 2 \mathrm{w}, \mathrm{T} 2 \mathrm{w}, \mathrm{rho} 2 \mathrm{wake}, \mathrm{w} 2 \mathrm{w}]$; $\frac{\circ}{\circ}$

Constructs the output vector and retuns nessesary quantities to main.m

\section{Mixcalc.m}

\% This is the final calculation performed in the impeller two-zone model.

등 this point, data can be passed to the vaneless diffuser algorithm if

o data regarding the diffuser is available. The mixed-out state requires

o iteration on the mixed-out stagnation temperature utilizing several

o external loss correlations for determining the recirculation loss,

tip

o leakage loss and disc friction loss. The mixed-out calculation scheme is

\% heavily based upon the work performed by oh et al. (2002) with several

$\%$ equations taken directly from their model. Inputs to this part of the

o model encompass much of the data generated by the jet and wake zone

o models to this point and include: wake mass flow fraction, absolute

$\frac{0}{\partial}$ tangential velocity of the jet, exit tangential velocity of the wake, jet

o exit stagnation temperature, wake exit stagnation temperature, mass

flow

o rate, outlet radius, outlet blade height, specific heat ratio, universal

o gas constant, impeller geometric area, jet static pressure, jet

o meridional velocity, wake meridional velocity, jet flow angle, blade

o angle, impeller exit tangential velocity, jet relative velocity, wake

o relative velocity, inlet tip relative velocity, inlet tip radius,

o equivanlent number of blades, inlet static density, inlet axial

velocity

$\frac{\circ}{\partial}$ and inlet hub radius.

function output $=$ mixcalc $\langle$ chi, Ctheta2j, Ctheta2w, T02j, T02w, mdot, r2, b2, gamma, R, Ageo, $\mathrm{P} 2 j, \mathrm{Cr} 2 j, \mathrm{Cr} 2 w$, beta2j, beta2blade, U2, W2j, w2w, w1t, r1t, $\mathrm{z}, \mathrm{rho1}, \mathrm{Cx} 1, \mathrm{r} 1 \mathrm{~h})$ 


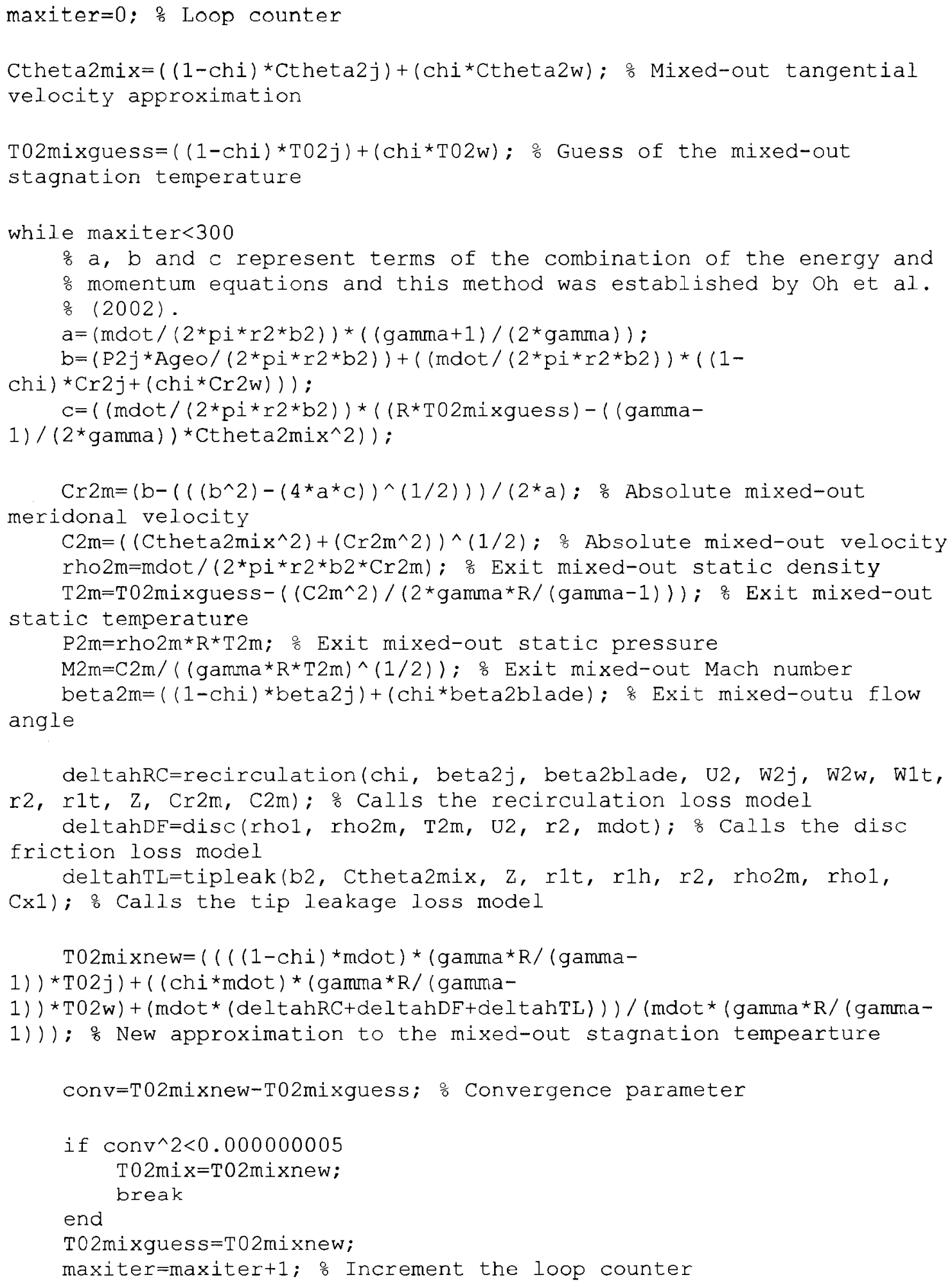

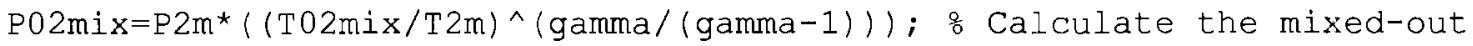
stagnation pressure based upon isentropic relationships 
output $=[\mathrm{P0} 2 \mathrm{mix}, \mathrm{T} 02 \mathrm{mix}, \mathrm{P} 2 \mathrm{~m}, \mathrm{~T} 2 \mathrm{~m}, \mathrm{rho} 2 \mathrm{~m}, \mathrm{C} 2 \mathrm{~m}$, beta2m]; 告 Establishes the output vector and returns any necessary quantities to main.m 


\section{Appendix B - Sample Input Two-Zone Model File}

\section{File Format}

Parameter

$\mathrm{P}_{01}$

$\mathrm{T}_{01}$

$\dot{m}_{\text {des }}$

$\mathrm{N}_{\text {des }}$

$\mathrm{r}_{1 \mathrm{~h}}$

$r_{1 t}$

$\mathrm{r}_{2}$

$b_{1}$

$b_{2}$

$\beta_{2 b l}$

$\mathrm{Z}_{\text {split }}$

$Z_{\text {full }}$

$t_{2 b}$

$\beta_{1 b l_{h}}$

$\beta_{1 b l_{t}}$

$\mathrm{PR}_{\mathrm{des}}$

\section{$\underline{\text { Units }}$}

$\mathrm{kPa}$

$\mathrm{K}$

$\mathrm{kg} / \mathrm{s}$

RPM

$\mathrm{mm}$

$\mathrm{mm}$

$\mathrm{mm}$

$\mathrm{mm}$

$\mathrm{mm}$

deg.

(none)

(none)

$\mathrm{mm}$

deg.

deg.

(none)

\section{Sample Input File}

The following input file is that used for the determination of the characteristics for the Eckhardt $\mathrm{O}$ impeller.

101
288
5.32
14000
45
140
200
95
26
0
20
0


3

63

63

2.15 


\section{Appendix C - Sample Beta Grid Input File}

The following appendix shows the format required for the input file into the beta grid definition file. This is used initially to perform the low speed extrapolation technique, but is then used in turn to perform the overall engine performance. Initially, a zero is found on the first line, as well as any line indicating a speed change. Following a zero start line, all the values of mass flow rate, pressure ratio and isentropic efficiency for that speed line are given. This process continues, starting with the lowest speed characteristic data continuing to highest speed characteristic.

\section{File Format}

$\begin{array}{ccc}0 & N_{\text {low }} & 0 \\ \dot{m} & P R & \eta \\ \dot{m} & P R & \eta \\ 0 & N & 0 \\ \dot{m} & P R & \eta \\ \dot{m} & P R & \eta \\ 0 & N_{\text {high }} & 0 \\ \dot{m} & P R & \eta\end{array}$

\section{Sample Input File}

$\begin{array}{lll}0 & 10000 & 0 \\ 2.306831683 & 1.49546 & 0.85678 \\ 2.407128713 & 1.49538 & 0.86282 \\ 2.507425743 & 1.49519 & 0.86733 \\ 2.607722772 & 1.49491 & 0.87063 \\ 2.708019802 & 1.49455 & 0.87295 \\ 2.808316832 & 1.49412 & 0.87451\end{array}$




\begin{tabular}{lll}
2.908613861 & 1.49364 & 0.87545 \\
3.008910891 & 1.49311 & 0.87592 \\
3.109207921 & 1.49254 & 0.87602 \\
3.209504951 & 1.49193 & 0.87582 \\
3.30980198 & 1.49129 & 0.8754 \\
3.41009901 & 1.49063 & 0.87479 \\
3.51039604 & 1.48994 & 0.87405 \\
3.610693069 & 1.48923 & 0.87319 \\
3.710990099 & 1.48851 & 0.87226 \\
3.811287129 & 1.48776 & 0.87126 \\
3.911584158 & 1.487 & 0.8702 \\
4.011881188 & 1.48623 & 0.86911 \\
4.112178218 & 1.48545 & 0.868 \\
4.212475248 & 1.48466 & 0.86686 \\
4.312772277 & 1.48386 & 0.86571 \\
4.413069307 & 1.48306 & 0.86454 \\
4.513366337 & 1.48225 & 0.86337 \\
4.613663366 & 1.48145 & 0.8622 \\
0 & 12000 & 0 \\
3.008910891 & 1.78432 & 0.86054 \\
3.109207921 & 1.78393 & 0.86459 \\
3.209504951 & 1.78339 & 0.86777 \\
3.30980198 & 1.78274 & 0.87022 \\
3.41009901 & 1.78198 & 0.87207 \\
3.51039604 & 1.78112 & 0.87341 \\
3.610693069 & 1.78019 & 0.87434 \\
3.710990099 & 1.77918 & 0.87491 \\
3.811287129 & 1.77811 & 0.8752 \\
3.911584158 & 1.77698 & 0.87524 \\
4.011881188 & 1.77581 & 0.8751 \\
4.112178218 & 1.77459 & 0.87479 \\
4.212475248 & 1.77333 & 0.87435 \\
4.312772277 & 1.77204 & 0.87379 \\
4.413069307 & 1.77072 & 0.87315 \\
4.513366337 & 1.76936 & 0.87244 \\
4.613663366 & 1.76799 & 0.87167 \\
4.713960396 & 1.76659 & 0.87085 \\
4.814257426 & 1.76517 & 0.86999 \\
4.914554455 & 1.76374 & 0.86909 \\
5.014851485 & 1.7623 & 0.86817 \\
5.115148515 & 1.76084 & 0.86723 \\
5.215445545 & 1.75937 & 0.86627 \\
5.315742574 & 1.7579 & 0.8653 \\
5.416039604 & 1.75643 & 0.86432 \\
0 & 14000 & 0 \\
3.911584158 & 2.16405 & 0.86568 \\
& & \\
\hline
\end{tabular}




$\begin{array}{lll}4.011881188 & 2.16284 & 0.86801 \\ 4.112178218 & 2.16147 & 0.86988 \\ 4.212475248 & 2.15996 & 0.87135 \\ 4.312772277 & 2.15832 & 0.87247 \\ 4.413069307 & 2.15657 & 0.8733 \\ 4.513366337 & 2.15471 & 0.87387 \\ 4.613663366 & 2.15277 & 0.87423 \\ 4.713960396 & 2.15074 & 0.87439 \\ 4.814257426 & 2.14864 & 0.8744 \\ 4.914554455 & 2.14647 & 0.87427 \\ 5.014851485 & 2.14424 & 0.87402 \\ 5.115148515 & 2.14195 & 0.87367 \\ 5.215445545 & 2.13962 & 0.87323 \\ 5.315742574 & 2.13724 & 0.87272 \\ 5.416039604 & 2.13482 & 0.87215 \\ 5.516336634 & 2.13237 & 0.87153 \\ 5.616633663 & 2.12988 & 0.87086 \\ 5.716930693 & 2.12671 & 0.87006 \\ 5.817227723 & 2.12411 & 0.86931 \\ 5.917524753 & 2.12148 & 0.86854 \\ 6.017821782 & 2.11883 & 0.86774 \\ 6.118118812 & 2.11616 & 0.86692 \\ 6.218415842 & 2.11348 & 0.86608 \\ 0 & 16000 & 0 \\ 4.914554455 & 2.65126 & 0.86799 \\ 5.014851485 & 2.64861 & 0.86947 \\ 5.115148515 & 2.64578 & 0.87066 \\ 5.215445545 & 2.64277 & 0.87161 \\ 5.315742574 & 2.63961 & 0.87235 \\ 5.416039604 & 2.63631 & 0.87289 \\ 5.516336634 & 2.63288 & 0.87326 \\ 5.616633663 & 2.62933 & 0.87348 \\ 5.716930693 & 2.62482 & 0.8735 \\ 5.817227723 & 2.62097 & 0.87346 \\ 5.917524753 & 2.61702 & 0.87332 \\ 6.017821782 & 2.61298 & 0.87309 \\ 6.118118812 & 2.60886 & 0.87279 \\ 6.218415842 & 2.60465 & 0.87241 \\ 6.318712871 & 2.60038 & 0.87197 \\ 6.419009901 & 2.59605 & 0.87148 \\ 6.519306931 & 2.59165 & 0.87094 \\ 6.61960396 & 2.5872 & 0.87036 \\ 6.71990099 & 2.5827 & 0.86974 \\ 6.82019802 & 2.57816 & 0.8691 \\ 6.92049505 & 2.57358 & 0.86842 \\ 7.020792079 & 2.56897 & 0.86772\end{array}$

